OAK RIDGE NATIONAL LABORATORY
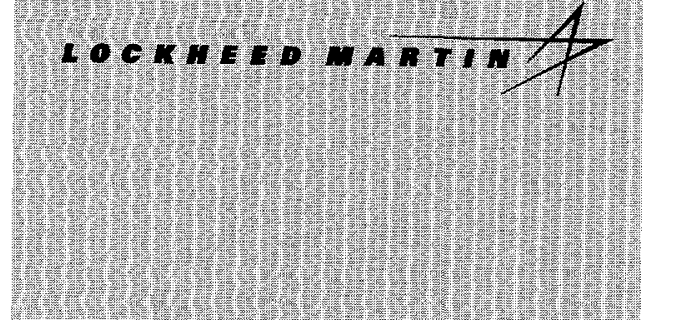

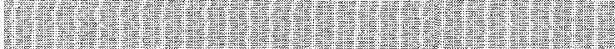

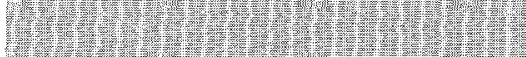

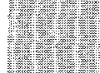
IIt) 12:

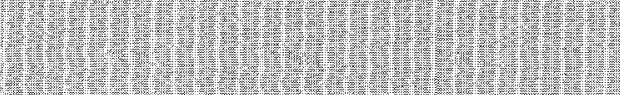
1.

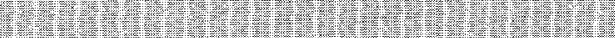
- The

WANAEED ANO OPERATEO BY LOCKAEED WARTW ENERGY RESEARCH CORPORATION FOA THE UNTED STATES

DEPARTIEMT OF ENERGY

\title{
Analysis of Errors in a Special Perturbations Satellite Orbit Propagator
}

J. P. Jones

M. Beckerman
RECEIVED

MAR 021999

OSTI 
This report has been directly reproduced from the best available copy.

Available to DOE and DOE contractors from the Office of Scientific and Technical Information, P.O. Box 62, Oak Ridge, TN 37831; prices from (423) 576-8401.

Available to the public from the National Technical Information Service, U.S. Department of Commerce, 5285 Port Royal Rd., Springfield, VA 22161.

This report was prepared as an account of work sponsored by an agency of the United States Government. Neither the United States nor any agency thereof, nor any of their employees, makes any warranty, express or implied, or assumes any legal liability or responsibility for the accuracy, completeness, or usefulness of any information, apparatus, product, or process disclosed, or represents that its use would not infringe privately owned rights. Reference herein to any specific commercial product, process, or service by trade name, trademark, manufacturer, or otherwise, does not necessarily constitute or imply its endorsement, recommendation, or favoring by the United States Government or any agency thereof. The views and opinions of authors expressed herein do not necessarily state or reflect those of the United States Government or any agency thereof. 


\section{DISCLAIMER}

Portions of this document may be illegible in electronic image products. Images are produced from the best available original document. 
Computer Science and Mathematics Division

\title{
ANALYSIS OF ERRORS IN A SPECIAL PERTURBATIONS SATELLITE ORBIT PROPAGATOR
}

J. P. Jones and M. Beckerman

Date Published: February 1999

\author{
Prepared by the \\ OAK RIDGE NATIONAL LABORATORY \\ Oak Ridge, Tennessee 37831 \\ Managed by \\ LOCKHEED MARTIN ENERGY RESEARCH CORP. \\ for the \\ U.S. DEPARTMENT OF ENERGY \\ under Contract No. DE-AC05-96OR22464
}




\begin{abstract}
We performed an analysis of error densities for the Special Perturbations orbit propagator using data for 29 satellites in orbits of interest to Space Shuttle and International Space Station collision avoidance. We find that the along-track errors predominate. These errors increase monotonically over each 36-hour prediction interval. The predicted positions in the along-track direction progressively either leap ahead of or lag behind the actual positions. Unlike the along-track errors the radial and cross-track errors oscillate about their nearly zero mean values. As the number of observations per fit interval decline the along-track prediction errors, and amplitudes of the radial and cross-track errors, increase.
\end{abstract}

In the low earth orbit regime (eccentricities $<0.2$ and perigees $<450 \mathrm{~km}$ ) the root-mean-square (rms) errors in the along-track direction vary from $200 \mathrm{~m}$ to over $2 \mathrm{~km}$ at 36 hours prediction time. The radial and cross-track errors are smaller than the along-track errors by roughly an order of magnitude. The cross-track errors remain nearly constant over the 36-hour prediction intervals while the radial error amplitudes increase.

Systematic errors arising from unmodeled drag forces are present in the error profiles for all low earth orbit satellites. The along-track error growth is correlated with the decay rate of the satellite semi-major axis. Since the decay of the semi-major axis is a consequence of non-conservative drag forces this correlation serves to link the systematic along-track errors to inadequacies in the atmospheric drag model and its parameters.

Progressively larger ephemeris prediction errors along all axes of motion are encountered as the satellite orbits become more eccentric. In the highly eccentric orbit regime (eccentricities $>0.2$ ) the rms errors in the along-track direction vary from $3 \mathrm{~km}$ to $9 \mathrm{~km}$ at 36 hours. The comparable errors in the radial direction range from $500 \mathrm{~m}$ to almost $3 \mathrm{~km}$ while those in the cross-track direction are about a factor of three smaller. In the low earth orbit regime we find that the radial error growth rate is correlated with the mean eccentricity of the orbit. In the low earth orbit regime the eccentricities are small, and radial errors are far less than the along-track errors. When the eccentricities are large we observe radial errors on the order of several kilometers. There is a pronounced orbit wobble in this regime.

In the near circular orbit regime orbit (eccentricities $<0.015$ and perigees from $700 \mathrm{~km}$ to $1500 \mathrm{~km}$ ) the decline in prediction errors associated with a decreasing importance of unmodeled drag forces is offset by increasing uncertainties in the sensor data. In situations where the quality and quantity of data are low error spikes appear. These spikes, defined as errors of more than five standard deviations, are most pronounced in this regime, but appear in other regimes too whenever the data are degraded.

The magnitudes of the along-track errors, and the amplitudes of the radial and cross-track errors, are modulated in a random way by stochastic atmospheric effects and by random variations in the quantity of observational data. In the low earth orbit regime we find that the errors become Gaussian. In the other orbit regimes the ensembles of errors fail the test for Gaussian behavior due to the presence of shot-noiselike error spikes. 


V

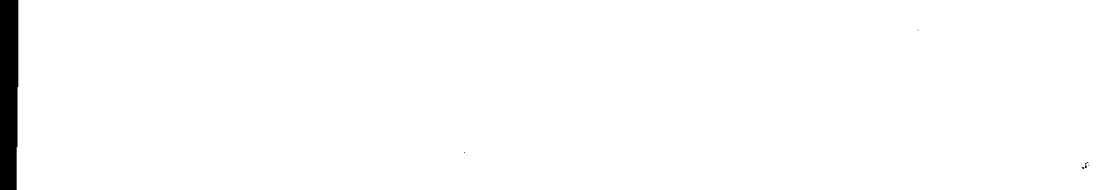

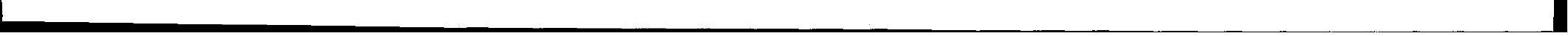

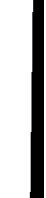

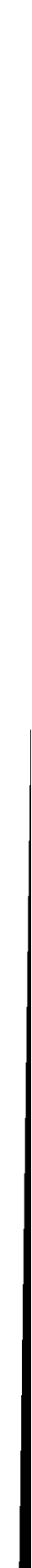

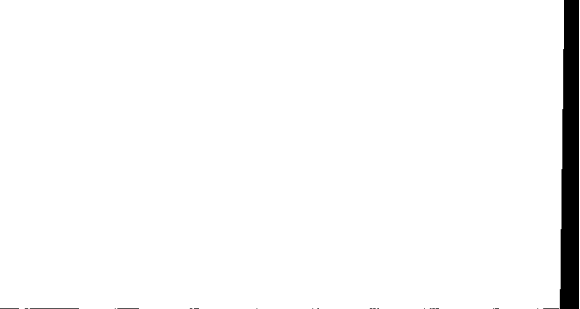




\section{TABLE OF CONTENTS}

Page
1.

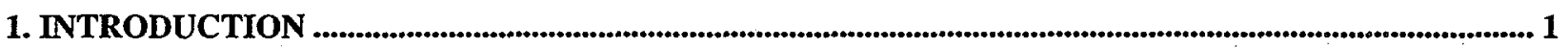

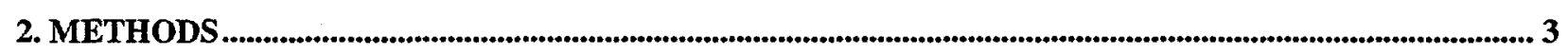

2.1 DATA

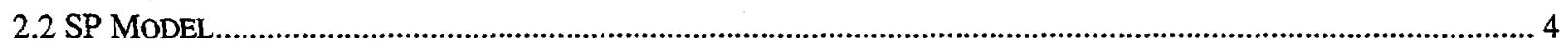

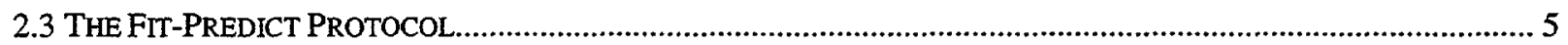

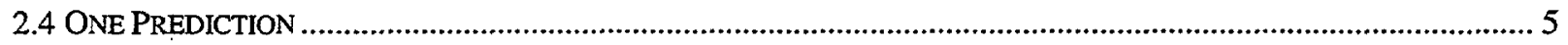

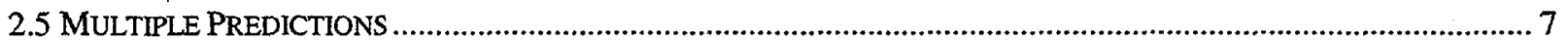

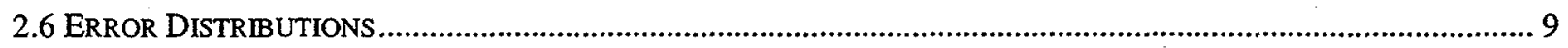

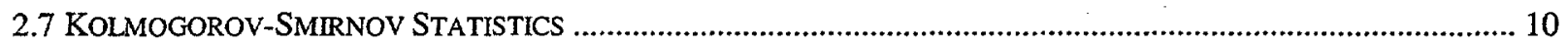

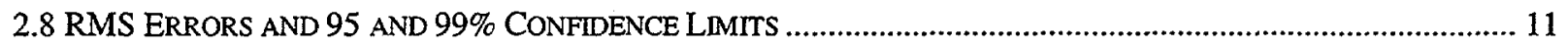

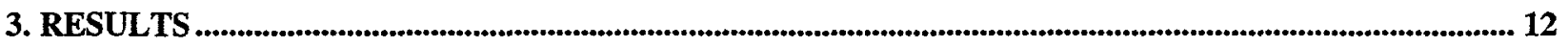

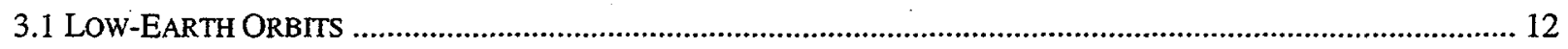

3.1.1 Error Profiles and Standard Deviations ........................................................................................... 12

3.1.2 Statistical Properties of Error Densities......................................................................................... I5

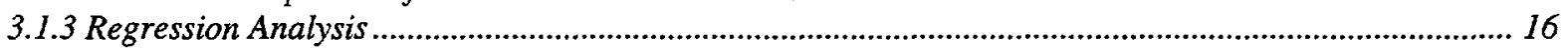

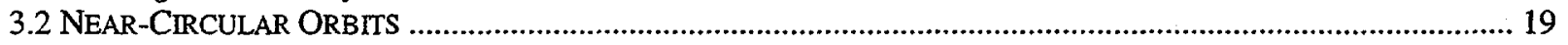

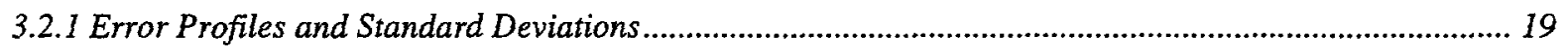

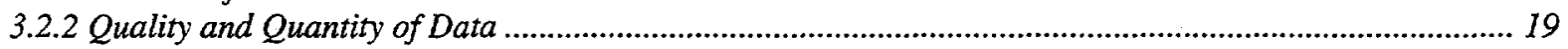

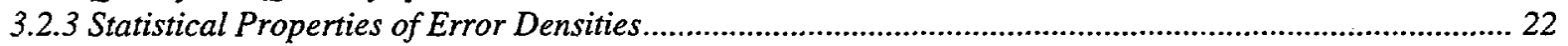

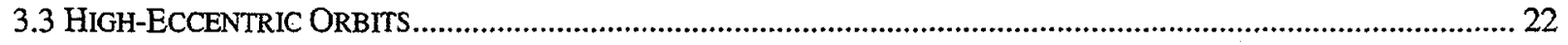

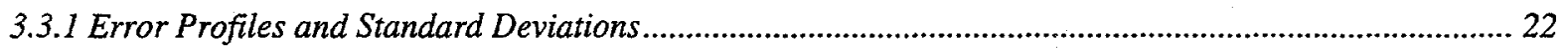

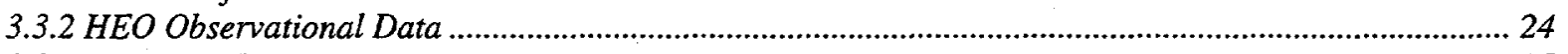

3.3.3 Statistical Properties of Error Densities .................................................................................................. 25

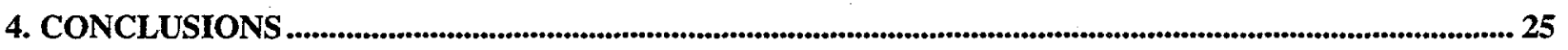

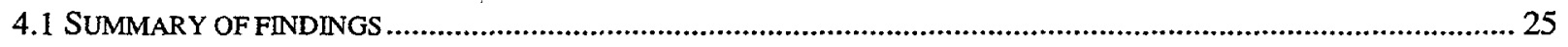

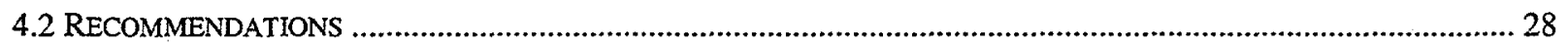

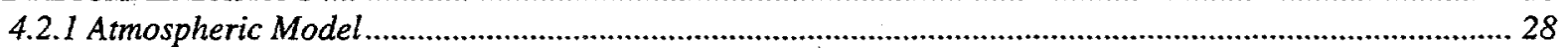

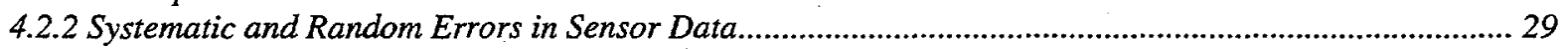

4.2.3 Stability, Model Selection, and Parameterization................................................................................... 29

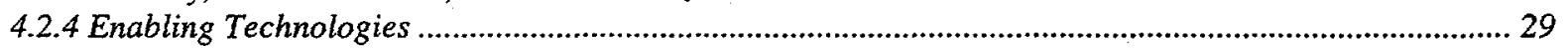

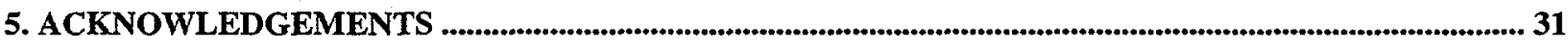

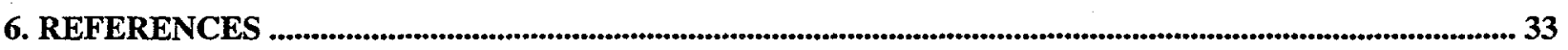

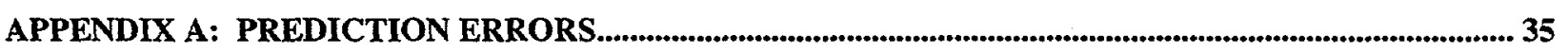

APPENDIX B: RMS ERRORS AND 95 AND 99\% CONFIDENCE LIMITS ....................................................67 


\section{LIST OF FIGURES}

Figure

Page

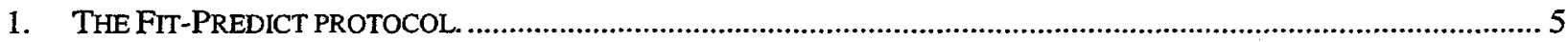

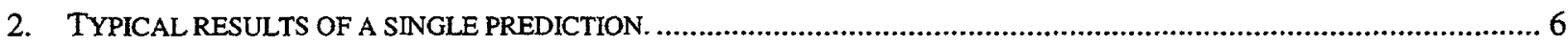

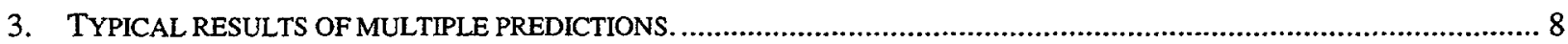

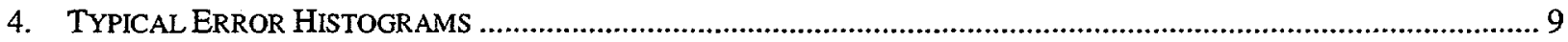

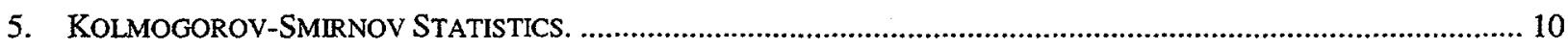

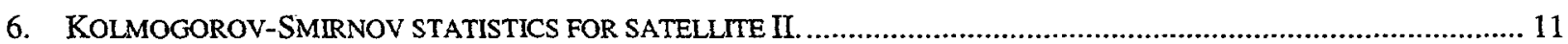

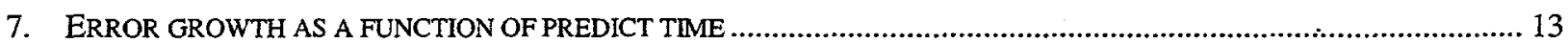

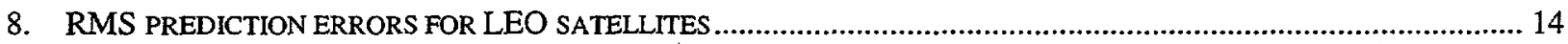

9. RADIAL PREDICTION ERRORS FOR 15 OF THE 19 LEO SATELLITES .............................................................. 15

10. KOLMOGOROV-SMIRNOV STATISTICS FOR THE COMBINED SAMPLE OF LEO.SATELLITES .................................. 17

11. CORRELATION OF SEMIMAJOR AXIS DECAY RATE AND ALONG-TRACK ERROR GROWTH RATE ............................. 18

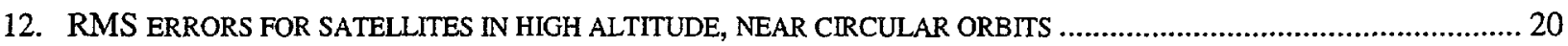

13. KOLMOGOROV-SMIRNOV STATISTICS FOR THE COMBINED SAMPLE OF NCO SATELLITES ................................... 21

14. RMS ERRORS FOR SATELLITES WITH HIGH ECCENTRICITY ORBITS...................................................................... 23

15. RELATIONSHIP BETWEEN DENSITY OF OBSERVATIONS AND ERROR MAGNITUDE.............................................. 24

16. KOLMOGOROV-SMIRNOV STATISTICS FOR THE COMBINED SAMPLE OF HEO SATELLITES....................................26 


\section{INTRODUCTION}

The increasing population of debris in low-earth orbit [1] has given rise to a collision hazard for orbiting assets. NASA, the U.S. Air Force, and other spacefaring agencies are consequently interested in accurate ephemeris prediction, and in quantification of prediction errors to provide a rational basis for collision avoidance procedures [2]. In this study we develop methods for estimating the distribution of errors in the predicted positions of a population of low-earth orbiting satellites, and model the rate at which these errors grow as a function of predict time.

The issue of error densities for an orbit propagator is complex. The errors one observes are determined not only by the selection of a model of the perturbations and the associated method of solution but also by the quality and quantity of the data and the physical properties of the satellites and their orbits. In order to attach meaning to the error densities one needs to tie their behavior to the underlying mechanisms that generate them. One has to understand the model's validity range, and the ensemble of random and systematic errors arising from unmodeled perturbations and uncertainties in the physical parameters of the force model. All of these quantities are interrelated and are not easy to untangle.

Our goal in this project was to determine the error densities for the Special Perturbations (SP) satellite orbit propagator and to construct a physical model of its evolution in time from 0 to 36 hours. To accomplish this goal we used a Unix Workstation version of the Goddard Trajectory Determination System (GTDS) [3,4] to perform the differential correction (DC) processes and to generate the ephemerides for satellites in a variety of orbits. This workstation version of GTDS was developed at the Draper Laboratory, has been used in previous space surveillance studies $[7,8,9]$, and is unique in that it can access a variety of numerical (SP), analytical (GP), and semi-analytical orbit propagators.

We have also made direct use of methods for initializing DCs and sequencing successive DCs, as well as methods for the manipulation of observational data, developed in previous studies [7, 8, 9]. In this study, observational data for 110 satellites acquired by the US Space Surveillance Network under a high level tasking protocol were examined. These data spanned two study intervals, one starting in October 1993 and the other beginning in December 1995. Both the data and the aforementioned methods were developed by and supplied courtesy of ITT Corporation.

In this report we present results of an in-depth analysis of the SP error densities for 29 satellites. These satellites were divided into three groups - Low Earth Orbit (LEO), Near Circular Orbit (NCO) and Highly Eccentric Orbit (HEO). Included in the first group were those satellites with eccentricities of less than 0.2 and perigees below $450 \mathrm{~km}$. The second group included satellites in near circular orbits (eccentricities of less than 0.015 ) and perigees from $700 \mathrm{~km}$ to $1500 \mathrm{~km}$. The third group consisted of those satellites that were in highly eccentric orbits, namely those with eccentricities greater than 0.2 . These satellites have perigees far into the thermosphere. Table 1 contains a summary of the orbit characteristics for the 29 satellites.

In our study we attempted to unravel and elucidate the networks of relationships above. The satellite groupings and the report are organized in a way that reflects these efforts. We begin in Section 2 with a summary of the methods used in our analysis. One objective in this study was to establish a baseline for future work in satellite orbit propagators. Section 2 contains descriptions of the SP, truth orbits, and the satellite observation data used to establish this baseline. 
In the report we show how satellite error densities evolve in time up to thirty-six hours. We present error profiles, error histograms, rms errors and 95/99\% confidence limits for the along-track, cross-track, and radial axes of motion for satellites in each of the three groupings. We present results of a regression analysis that establishes a physical model of the error densities. We also link the errors in the various regimes to the quality and quantity of the observational data. These results are presented in Section 3. Data for the LEO satellites are presented and discussed in Section 3.1. Included in Section 3.1 is a discussion of the statistical properties of the errors as revealed by a Kolmogorov-Smimov analysis. Results for the NCO satellites are presented in Section 3.2 and those for the HEO satellites in Section 3.3. We summarize our findings in Section 4.1 and make recommendations in Section 4.2.

Table 1.

\begin{tabular}{|c|c|c|c|c|c|c|c|c|c|}
\hline $\begin{array}{l}\text { Satellite } \\
\text { Name }\end{array}$ & $\begin{array}{l}\text { Semimajor } \\
\text { Axis }(\mathrm{km})\end{array}$ & $\begin{array}{l}\text { Period } \\
\text { (min) }\end{array}$ & Eccentricity & $\begin{array}{c}\text { Inclination } \\
\text { (deg) }\end{array}$ & $\begin{array}{l}\text { Perigee } \\
(\mathrm{km})\end{array}$ & $\begin{array}{l}\text { Apogee } \\
(\mathrm{km})\end{array}$ & \#Runs ${ }^{*}$ & $\begin{array}{l}\text { Average } \\
\text { \#obs }\end{array}$ & Catagory \\
\hline$A A$ & 7971 & 118 & 0.0114 & 47 & 1502 & 1685 & 101 & 189 & NCO \\
\hline $\mathrm{BB}$ & 7228 & 101 & 0.0631 & 49 & 394 & 1305 & 73 & 186 & LEO \\
\hline $\mathrm{CC}$ & 7506 & 107 & 0.0070 & 50 & 1075 & 1182 & 76 & 282 & $\mathrm{NCO}$ \\
\hline DD & 9864 & 162 & 0.3119 & 60 & 416 & 6556 & 74 & 66 & $\mathrm{HEO}$ \\
\hline $\mathrm{EE}$ & 7499 & 107 & 0.0250 & 41 & 933 & 1308 & 105 & 287 & $\mathrm{NCO}$ \\
\hline $\mathrm{FF}$ & 7908 & 116 & 0.0176 & 56 & 1389 & 1670 & 106 & 222 & $\mathrm{NCO}$ \\
\hline GG & 7872 & 115 & 0.1380 & 144 & 407 & 2580 & 74 & 175 & LEO \\
\hline $\mathrm{HH}$ & 8563 & 131 & 0.2157 & 82 & 342 & 4028 & 70 & 105 & $\mathrm{HEO}$ \\
\hline II & 7612 & 110 & 0.1132 & 102 & 373 & 2095 & 73 & 234 & LEO \\
\hline JJ & 7674 & 111 & 0.1124 & 68 & 427 & 2164 & 73 & 227 & LEO \\
\hline $\mathrm{KK}$ & 7064 & 98 & 0.0323 & 62 & 438 & 933 & 73 & 182 & LEO \\
\hline LI & 7087 & 98 & 0.0504 & 73 & 354 & 1063 & 73 & 329 & LEO \\
\hline MM & 7335 & 104 & 0.0206 & 49 & 805 & 1107 & 76 & 248 & $\mathrm{NCO}$ \\
\hline NN & 6992 & 96 & 0.0268 & 29 & 426 & 802 & 73 & 176 & LEO \\
\hline 00 & 7269 & 102 & 0.0713 & 66 & 378 & 1402 & 70 & 352 & LEO \\
\hline PP & 7128 & 99 & 0.0499 & 66 & 394 & 1106 & 66 & 90 & LEO \\
\hline$Q Q$ & 7015 & 97 & 0.0482 & 27 & 299 & 974 & 73 & 197 & IEO \\
\hline $\mathrm{RR}$ & 13895 & 271 & 0.5172 & 26 & 330 & 14702 & 39 & 75 & HEO \\
\hline SS & 6773 & 92 & 0.0009 & 97 & 381 & 408 & 73 & 177 & LEO \\
\hline$T T$ & 6763 & 92 & 0.0026 & 31 & 367 & 403 & 73 & 170 & LEO \\
\hline UU & 7431 & 106 & 0.0857 & 82 & 412 & 1695 & 37 & 92 & IEO \\
\hline VV & 6802 & 93 & 0.0061 & 94 & 382 & 466 & 73 & 280 & LEO \\
\hline WW & 7703 & 112 & 0.1197 & 24 & 403 & 2247 & 74 & 188 & LEO \\
\hline $\mathrm{XX}$ & 7159 & 100 & 0.0001 & 98 & 771 & 790 & 16 & 262 & $\mathrm{NCO}$ \\
\hline$Y Y$ & 8123 & 121 & 0.1614 & 82 & 437 & 3051 & 73 & 298 & LEO \\
\hline $\mathrm{ZZ}$ & 9332 & 149 & 0.2850 & 41 & 297 & 5612 & 26 & 84 & $\mathrm{HEO}$ \\
\hline 11 & 6854 & 94 & 0.0051 & 35 & 443 & 513 & 73 & 183 & LEO \\
\hline 22 & 7539 & 108 & 0.1024 & 82 & 391 & 1931 & 73 & 353 & LEO \\
\hline 33 & 6762 & 92 & 0.0006 & 51 & 376 & 391 & 73 & 144 & IEO \\
\hline
\end{tabular}

${ }^{a}$ Number of 36-hour DCs' used to generate predicted ephemerides. 


\section{METHODS}

\subsection{Data}

We obtained, courtesy of ITT Corporation, data from the space surveillance network for approximately 110 satellites during two study intervals, one beginning in October 1993 and the other in December 1995. Other studies performed using these data include Refs. 5 through 7 . We used the Goddard Trajectory Determination System (GTDS) for performing DCs and generating ephemerides for selected satellites, and an automation system developed for this study for sequencing DCs. We also developed a number of computational and statistical tools for comparing orbits, calculating the statistics of error distributions and performing regressions, correlations, etc.

These satellites have semimajor axes in the range of 6762 to $13895 \mathrm{~km}$, periods in the range of 92 to $271 \mathrm{~min}$, eccentricities in the range of 0.0001 to 0.5172 , perigees in the range of 297 to $1502 \mathrm{~km}$, and apogees in the range of 391 to $14702 \mathrm{~km}$. Most of these satellites were selected because they (1) interact with the atmosphere in operationally interesting LEOs, (2) do not interact with the atmosphere, or (3) have highly eccentric orbits.

As mentioned in the introduction, the satellite data may be grouped several ways. We chose to group the data by their orbital characteristics, leading to designations LEO, NCO, and HEO. A second method of grouping data is by the quality and quantity of data used to compute an ephemeris. We discuss groupings according to this second method later in the text. We refer to Table 2, containing a summary of the density of observations, at several places in the report.

\section{Table 2}

General summary of the density of observations. The observation set for each satellite was divided into 36-hour periods. The minimum, maximum, and average number of observations for each period are listed, as is the RMS variation.

\begin{tabular}{lrrrr} 
Sat & MinObs & MaxObs & Avgobs & RMS \\
\hline AA & 54.00 & 276.00 & 189.64 & 56.25 \\
BB & 111.00 & 273.00 & 186.41 & 29.87 \\
CC & 73.00 & 432.00 & 282.92 & 70.99 \\
DD & 31.00 & 97.00 & 66.91 & 13.53 \\
EE & 56.00 & 420.00 & 287.04 & 71.52 \\
FF & 48.00 & 333.00 & 222.31 & 57.62 \\
GG & 79.00 & 246.00 & 175.54 & 32.41 \\
HH & 31.00 & 223.00 & 105.90 & 56.51 \\
II & 142.00 & 316.00 & 234.51 & 37.78 \\
JJ & 176.00 & 297.00 & 227.10 & 29.31 \\
KK & 120.00 & 249.00 & 182.19 & 28.41 \\
LI & 206.00 & 476.00 & 329.75 & 60.06 \\
MM & 89.00 & 420.00 & 248.25 & 67.46 \\
NN & 89.00 & 357.00 & 176.79 & 40.35 \\
OO & 252.00 & 449.00 & 352.07 & 45.15 \\
PP & 46.00 & 165.00 & 90.36 & 25.22 \\
QQ & 101.00 & 294.00 & 197.55 & 43.78 \\
RR & 25.00 & 130.00 & 75.87 & 25.95 \\
SS & 116.00 & 232.00 & 177.38 & 29.57 \\
TT & 64.00 & 269.00 & 170.84 & 37.73 \\
UU & 17.00 & 137.00 & 92.00 & 24.68 \\
VV & 211.00 & 361.00 & 280.21 & 27.75 \\
WW & 92.00 & 331.00 & 188.82 & 47.07 \\
XX & 209.00 & 324.00 & 262.00 & 26.14 \\
YI & 236.00 & 358.00 & 298.60 & 24.70 \\
ZZ & 35.00 & 123.00 & 84.85 & 25.44 \\
II & 102.00 & 270.00 & 183.36 & 36.49 \\
22 & 226.00 & 457.00 & 353.37 & 49.64 \\
33 & 97.00 & 195.00 & 144.75 & 23.22
\end{tabular}




\subsection{SP Model}

Ideally, one would like to account for a satellite's mass, drag, and reflectivity in the calculation of its orbit. However, many debris objects have unknown physical properties. Therefore, this information would not be available in a general setting. We have consequently assumed all satellites to be spherical, with a diameter of approximately $1.12 \mathrm{~m}$, a mass of $1.0 \mathrm{Kg}$, a reflectivity constant of 1.2 , and an area for radiation pressure of $1.0 \mathrm{~m}^{2}$.

All runs were processed using numerical integration of Cowell equations of motion. A $2 \times 0$ gravity field was used in numerical integration of cartesian state partials. Nonstate partials (drag and radiation) were also computed by numerical integration. All DCs were calculated using SP, SPADOC Jaccia-70 atmosphere, GEM 10B $21 \times 21$ geopotential, lunar-solar gravity, drag solved for, and solar radiation pressure applied. Normal equation solution by Choleski square-root normal matrix triangularization and double back-substitution was used for the least square. Other properties are listed in Table 3.

\section{Table 3.}

Numerical integration options and parameters, differential correction convergence parameters, force options considered, and physical constants used in the special perturbations model.

\section{NUMERICAL INTEGRATION OPTIONS AND PARAMETERS}

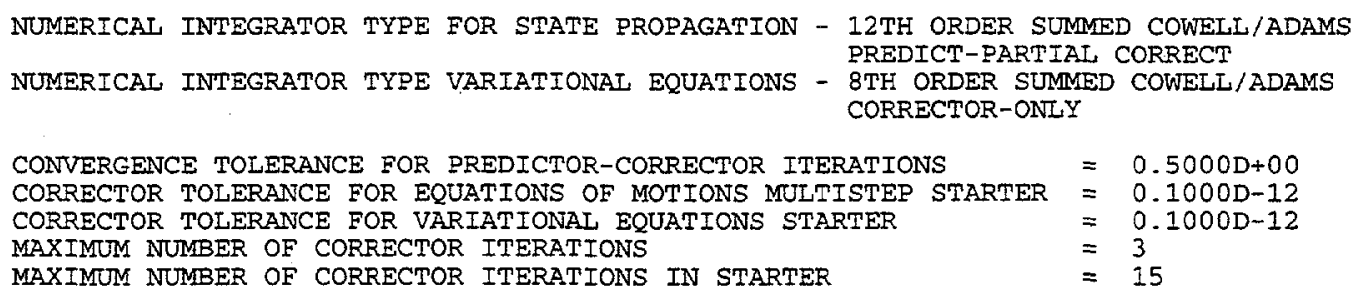

\section{DIFFERENTIAL CORRECTION CONVERGENCE PARAMETERS}

D.C. CONVERGENCE TOLERANCE

MINIMUM RMS FOR CONVERGENCE TEST

MAXIMUM NUMBER OF D.C. ITERATIONS

MAXIMUM NUMBER OF CONSECUTIVE DIVERGENT ITERATIONS $=3$

\section{FORCE OPTIONS CONSIDERED}

$=0.10000 \mathrm{D}-01$

$=0.30000 D-05$

29

SYSTEM OF INTEGRATION

NAME OF ATMOSPHERIC DENSITY MODEE

F10.7

P

NAME OF EARTH POTENTIAL FIELD MODEL

HARMONICS

MAX DEGREE

MAX ORDER

RESONANT POTENTIAL.

CENTRAL BODY

NON-CENT BODY1

NON-CENT BODY2

2-BODY MOTION

SOL RAD PRESS

$D R A G$

POLAR MOTION

\section{PHYSICAL CONSTANTS}

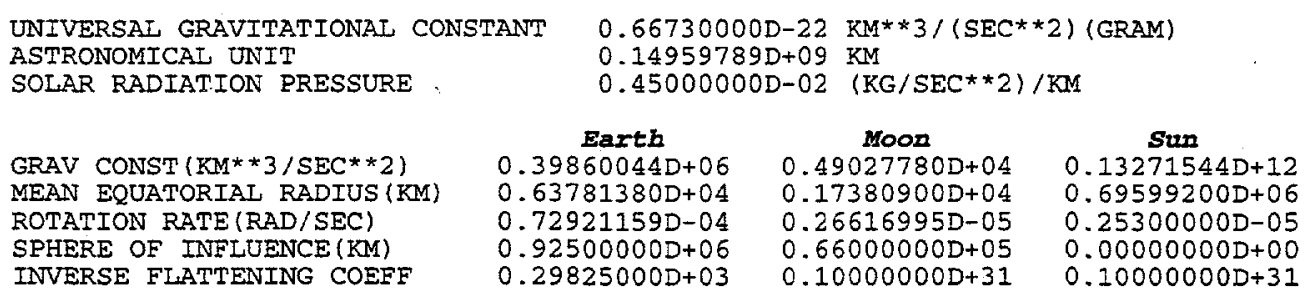




\subsection{The Fit-Predict Protocol}

To calculate the prediction error, for each satellite we ran differential corrections using data from successive 36-hour periods. For each of these DCs, 72-hour ephemerides were calculated at 3-minute intervals for both the 36-hour period covering the data (the fit interval) and the subsequent 36-hour period (the predict interval). Prediction errors for radial, cross-track, and along-track positions and velocities were determined by aligning corresponding segments from successive DCs and calculating the appropriate vector differences. Figure 1 illustrates this protocol.

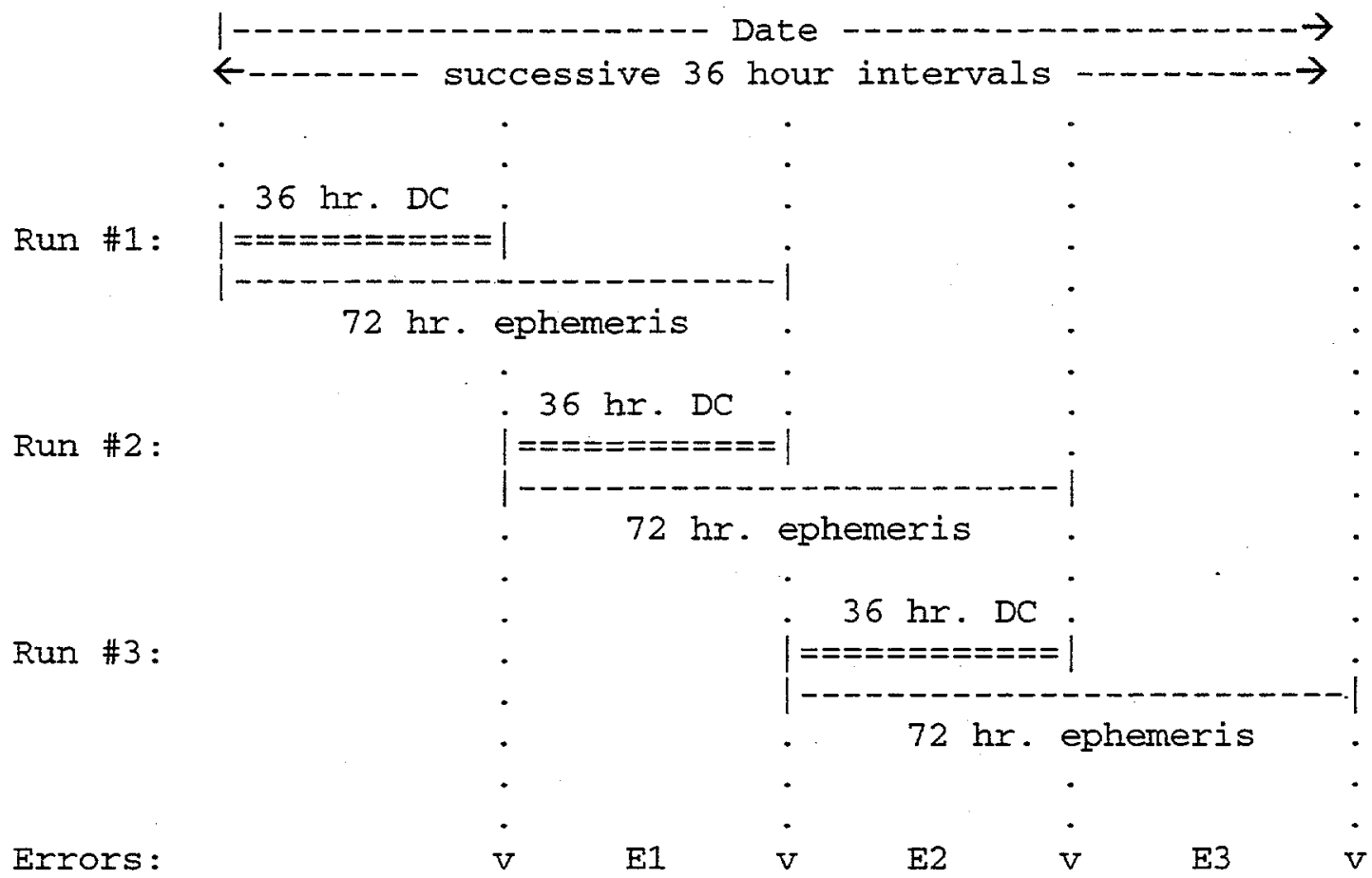

Fig. 1. The fit-predict protocol. Time proceeds from left to right. During each run, data from a 36hour period are used in a differential correction, but an ephemeris is generated over a 72-hour period. The difference between the ephemeris generated over the prediction interval and the ephemeris generated over the fit interval from the subsequent $D C$ are compared, producing an error ephemeris.

\subsection{One Prediction}

Figure 2 illustrates the results of a single ephemeris prediction. This satellite, designated II, is a lowearth orbiting object and is used subsequently for many examples. It has a semimajor axis of 7612-km, a 110 -minute period, a $373-\mathrm{km}$ perigee, and a $2095-\mathrm{km}$ apogee. Its eccentricity is 0.1132 . The data for this satellite are of high quality and are consist of an average of 234 observations per 36-hour interval throughout its study period.

The errors in Fig. 2 were created by taking the difference between the ephemeris predicted from a DC beginning Feb. 17, 1996 at 12:00 hours, extending through Feb. 19, 1996 at 00:00 hours, and an ephemeris fit to data collected beginning Feb. 19, 1996 at 00:00 hours, extending through Feb. 20, 1996 at 12:00 hours. The data for the first DC consisted of 277 observations collected in 61 runs from 16 sensors. Each run had an average of 4.5 observations. The data for the second DC consisted of 230 observations collected in 57 runs from 16 sensors. Each run had an average of 4.0 observations. Figure 2 illustrates the differences between the positions predicted by the first $\mathrm{DC}$ and the positions calculated by the second DC, given the second set of observations. 
A

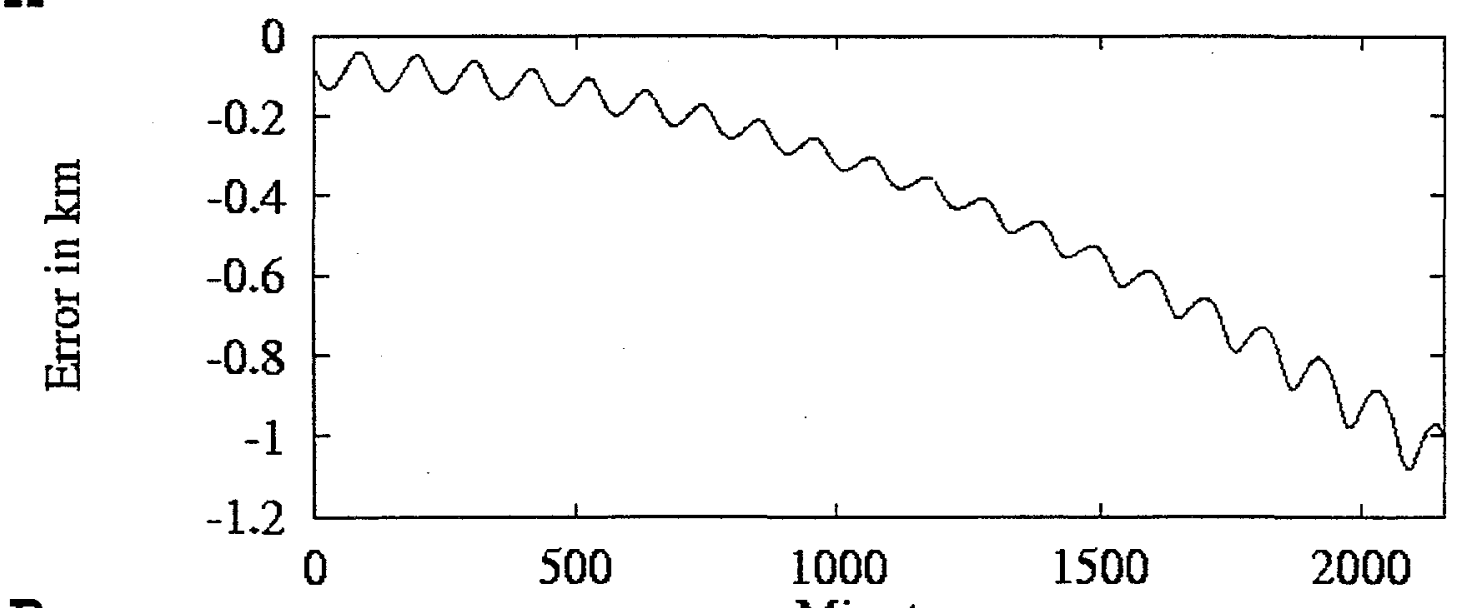

B

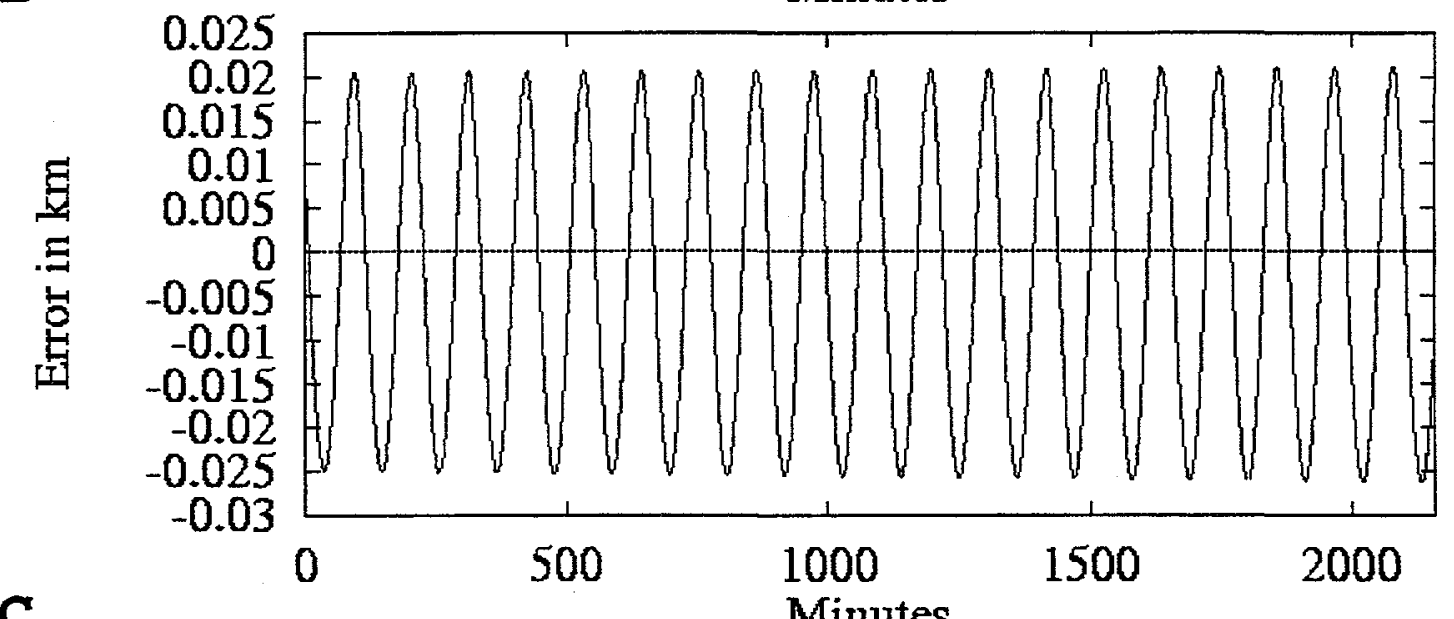

C

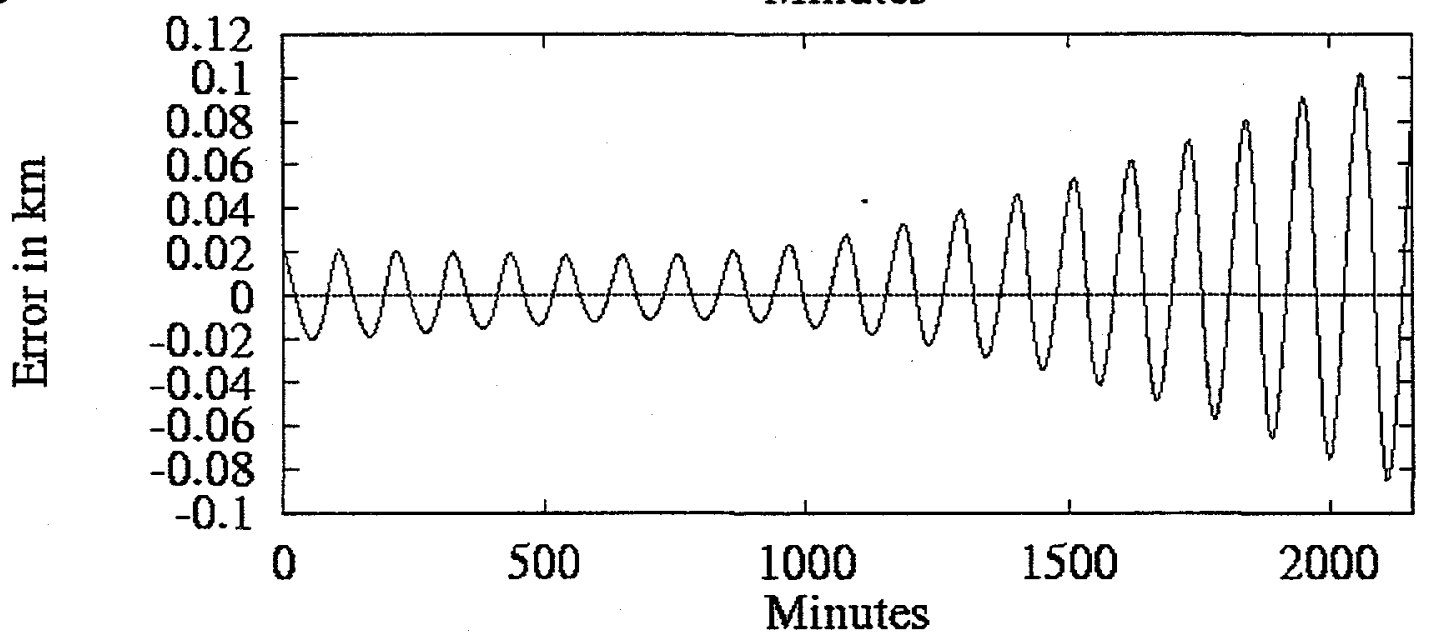

Fig. 2. Typical results of a single prediction, satellite II. (A) Along-track error. (B) Cross-track error. (C) Radial error. 
In many respects, the properties of the errors illustrated in Fig. 2 are typical of ephemeris prediction errors. Conspicuous in the error plots for all axes is an oscillation with a period corresponding to the orbital period of the satellite. However, in each plot this oscillation appears to be modified in characteristic ways.

Figure $2 \mathrm{~A}$ illustrates errors in the along-track direction. The difference between the predicted and observed positions is close to zero at predict time zero but, discounting the oscillation, monotonically decreases as predict time increases, to around $-1 \mathrm{~km}$. This indicates that the predicted position of the satellite lagged behind the subsequently observed position. Note that the magnitude of the lag increases faster than linearly. This faster-than-linear increase, while not always observed, appears to be a governing feature of this category of error.

Figure 2B illustrates errors in the cross-track direction. In this case, the oscillation remains about zero. Its amplitude appears to neither grow nor shrink by a significant amount as predict time increases, nor does its mean drift significantly away from zero. This behavior indicates that the estimation of the satellite's inclination changed a small amount between the two DCs: the amplitude of the oscillation is 20 to $25 \mathrm{~m}$. A small amplitude oscillation, which does not change as a function of predict time, is characteristic of this category of error.

Figure $2 \mathrm{C}$ illustrates error in the radial direction. As in the previous case, the oscillation appears to remain about zero, but its amplitude changes as predict time increases, remaining approximately constant during the first half of the predict interval, then increasing near linearly during the second half of the predict interval. The amplitude of the oscillation begins at about $20 \mathrm{~m}$ and increases to about $100 \mathrm{~m}$. While it is characteristic for this category of error to oscillate around zero, the way in which the amplitude behaves as a function of predict time is generally complex.

\subsection{Multiple Predictions}

Figure 3 illustrates the results of 20 ephemeris predictions. Again, we use satellite II as an example. These predictions are the result of successive DCs run using data collected during February 1996. (For comparison, the data in Fig. 2 correspond to the data for prediction 12 in Fig. 3.)

Inspection of Fig. 3 reveals some of the properties of ensembles of prediction errors. Figure 3A illustrates 20 along-track prediction errors. Most conspicuously, although the underlying curves almost all uniformly drift away from zero, they differ in both magnitude and sign. This indicates that the predictions either lagged or led the satellite's position as determined from subsequent observations and that they appear to do so by some random amount. The faster-than-linear growth rate appears to be present in most of the errors; prediction number 6 stands as an obvious counterexample.

Figure 3B illustrates errors in the cross-track direction. As in the case illustrated previously, the mean of each oscillation remains about zero, with the amplitude changing little as predict time increases. This amplitude, however, differs from prediction to prediction, reaching a maximum of about $60 \mathrm{~m}$ for prediction 5 and a minimum of less than $10 \mathrm{~m}$ for prediction 11.

Figure $3 \mathrm{C}$ illustrates errors in the radial direction. As in the previous case, each oscillation remains about zero, with its amplitude generally, though not always, increasing as a function of predict time. The peaks of these oscillations, achieved after 36 hours, have amplitudes between the along-track and crosstrack directions, ranging from near 0 to about $150 \mathrm{~m}$. Again, there appears to be little if any consistent pattern governing error amplitudes between trials.

Although these examples contain many of the characteristic features of ephemeris prediction errors, they by no means illustrate the entire range of behaviors. Appendix A contains concatenated prediction errors for all of the satellites analyzed. 
A
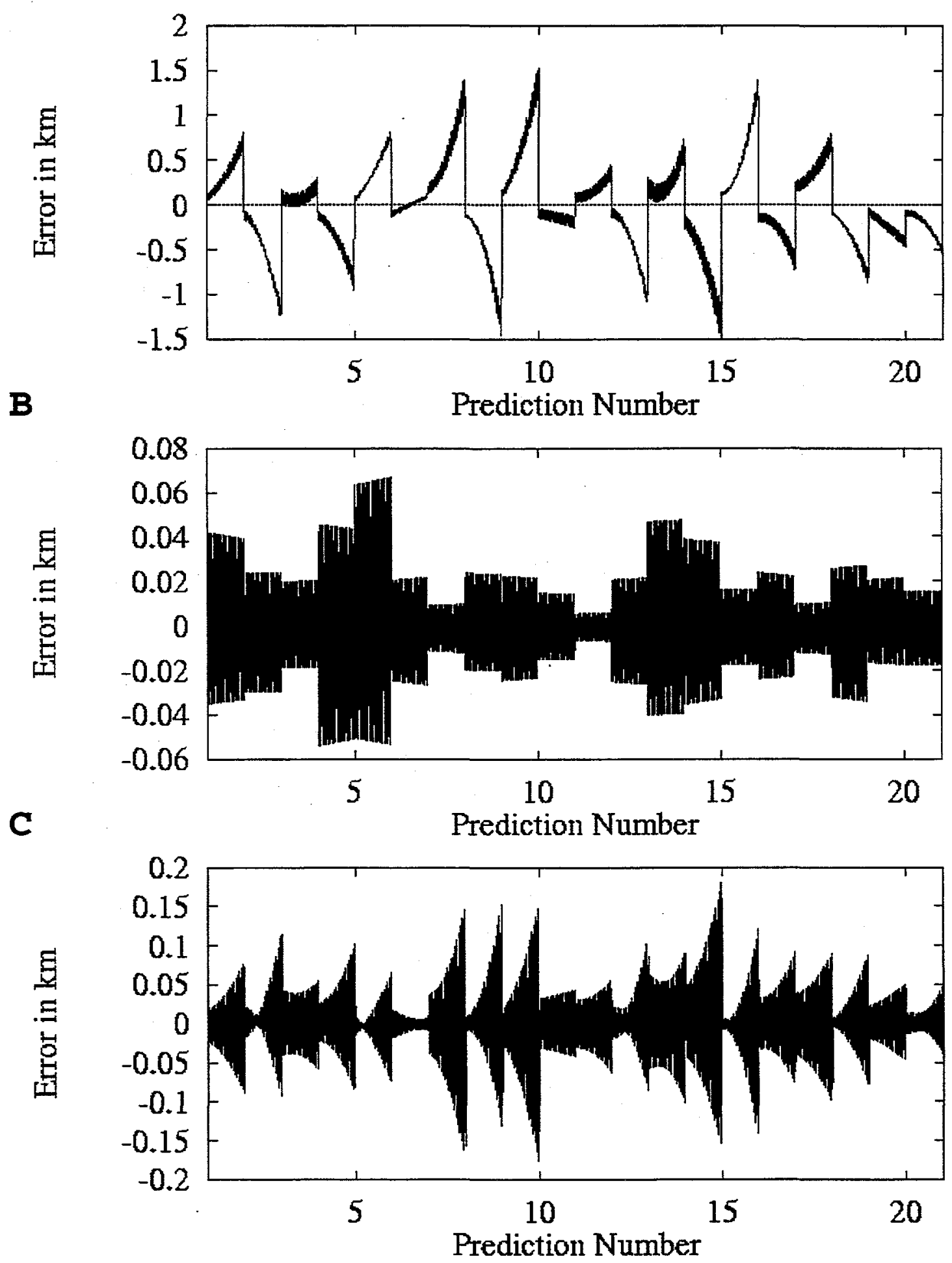

Fig. 3. Typical results of multiple predictions, satellite II. (A) Along-track errors. (B) Cross-track errors. (C) Radial errors. 


\subsection{Error Distributions}

To calculate the distribution of prediction errors for a satellite, we accumulate a selected set of errors from all predictions for that satellite. To create this set, we begin with the error at the time of interest. To eliminate the effects of the oscillations from the determination of error, we also include in the set errors for one orbital period following the time of interest. This procedure allows us to determine the distribution of errors during the one orbital period following a specified time. These distributions formed the basis for subsequent analyses.

Figure 4 illustrates error distributions for satellite II at predict times of $0,12,24$, and 36 hours for along-track, cross-track, and radial directions. Most of the distributions are prominently peaked near $0 \mathrm{~km}$. In the along-track direction, the distributions grow conspicuously as predict time increases: the distribution at time 0 hours is well contained within $+/-0.5 \mathrm{~km}$, whereas the distribution at predict time 36 hours spreads to slightly over $+/-1.5 \mathrm{~km}$. The radial error distributions exhibit a similar pattern of growth. In this case the distribution spreads from $+/-0.05$ to $+/-0.2 \mathrm{~km}$ as predict time increases. In contrast, the distributions of along-track error do not appear to grow with predict time, remaining roughly constant at $+/-0.05 \mathrm{~km}$ throughout. The growth of the distributions of along-track and radial errors while the crosstrack errors remain constant reflects the character of the underlying errors illustrated previously.
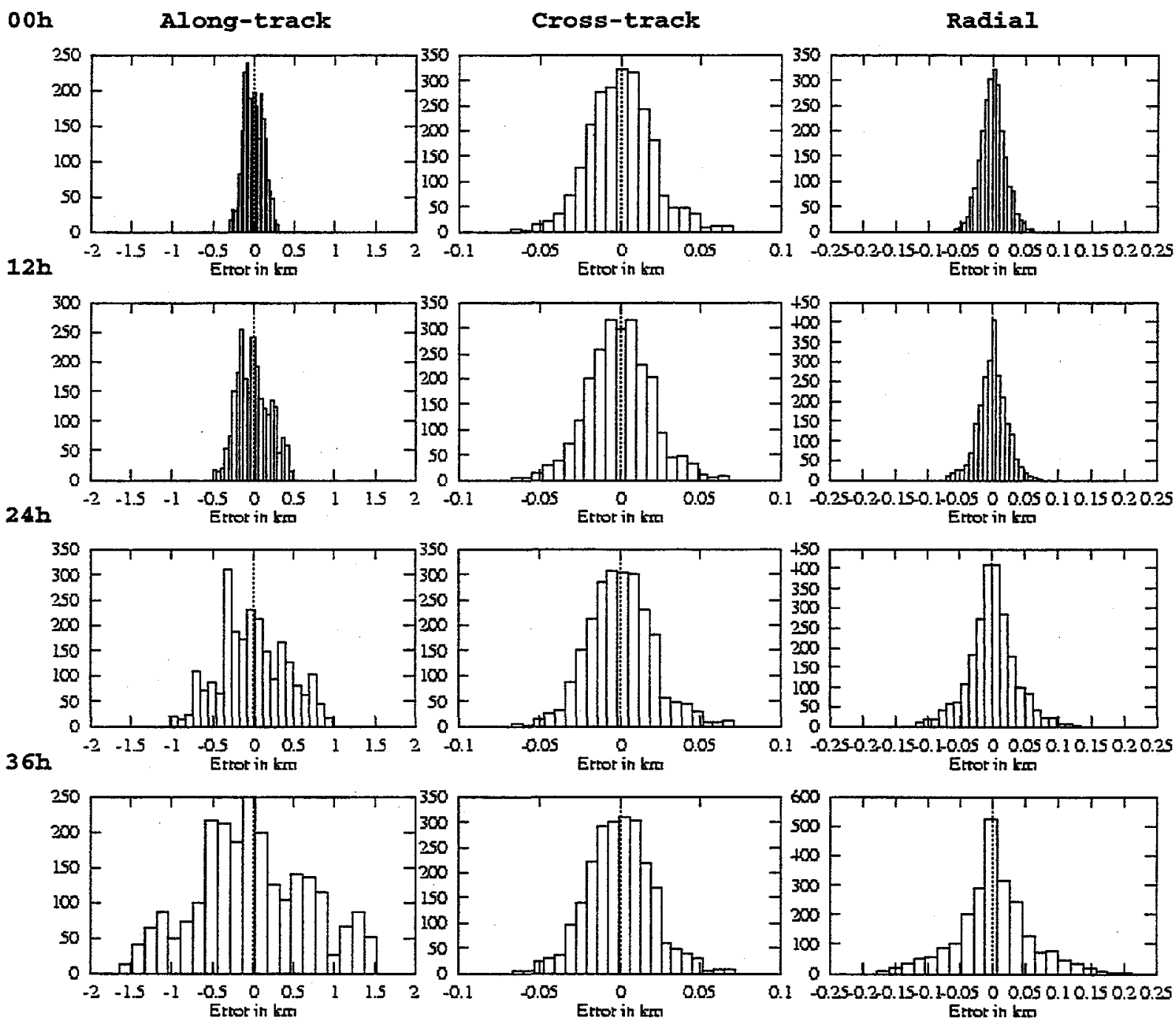

Fig. 4. Error histograms for along-track, cross-track, and radial errors at 0, 12,24, and 36 hours. 


\subsection{Kolmogorov-Smirnov Statistics}

The Kolmogorov-Smirnov (K-S) statistic provides a technique for constructing a confidence band for a distribution. It also provides a method for testing hypotheses concerning the shape of a distribution, that is, whether or not the data are consistent with some a priori model of their distribution. In this section, we consider whether or not the distributions constructed in the previous section are consistent with a normal distribution.

Figure 5 illustrates the construction. By extracting individual errors from a set of predictions, a sample of errors has been accumulated. These errors are normalized to the standard coordinate $z=x-\mu / \sigma$. The cumulative distribution of the sample, $S_{n}(x)$, is illustrated in Fig. 5 by a solid line. By adding and subtracting a critical value $D_{n}{ }^{\alpha}$ from $S_{n}(x)$, we form confidence limits for the sampled data, illustrated in Fig. 5 by dashed lines above and below the solid line. The number of samples in the population and the confidence coefficient 1- $\alpha$ determines the distances of these confidence limits from the data. For the present analyses, we are motivated to construct conservative tests that reject the null hypothesis as easily as possible. Consequently, all of the confidence limits illustrated in this report used $\alpha=0.2=$ $1.07 / \mathrm{sqrt}(\mathrm{N})$. This is also referred to as an $80 \%$ confidence limit. Confidence limits constructed in this manner provide the greatest opportunity for our a priori notions regarding the distribution of data to be demonstrated incorrect.

Specifically, given the large number of samples present, one may naturally expect the errors to be normally distributed. The dashed curve coursing through the center of Fig. 5 is the cumulative distribution function of the standard normal distribution. It is clear in this example that although the distribution function of the standard normal distribution resides largely above the data on the left side of the figure, and below on the right side, nowhere does it deviate from the data by an amount sufficient to intersect the confidence limits. From this observation we are able to conclude that there is insufficient evidence in this sample to reject the hypothesis that the data are normally distributed. In other words, the data are consistent with the hypothesis that they are normally distributed.

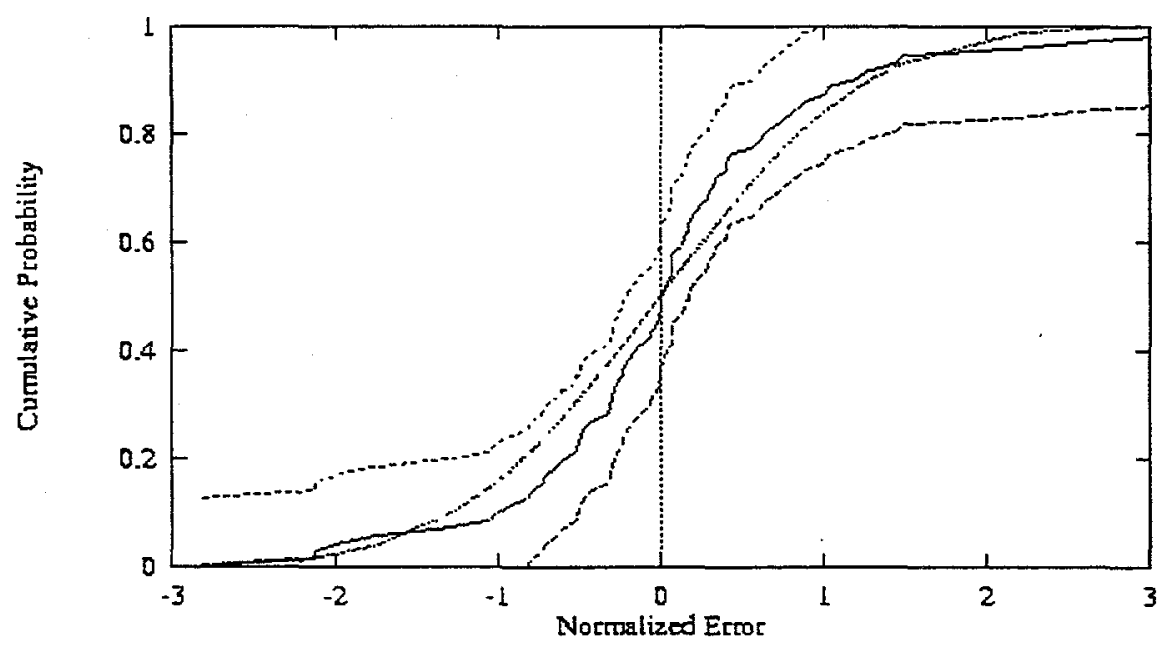

Fig. 5. K-S statistics for satellite II, radial prediction errors at 36 hours. 
$\mathrm{OOh}$ Along-Track Cross-track Radial

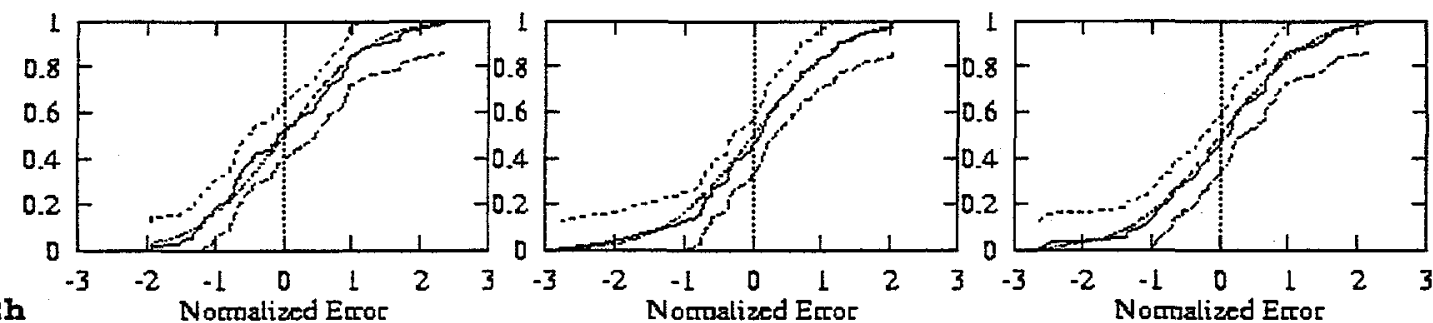

$12 \mathrm{~h}$
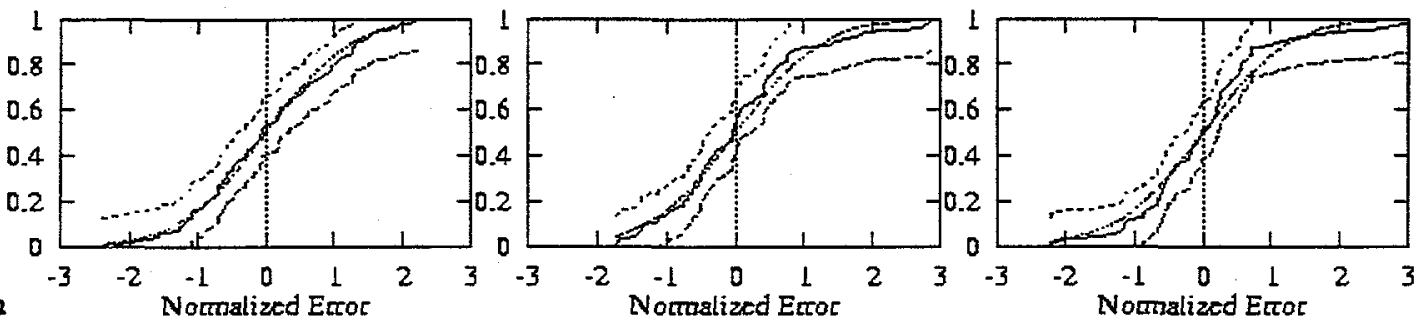

$24 h$
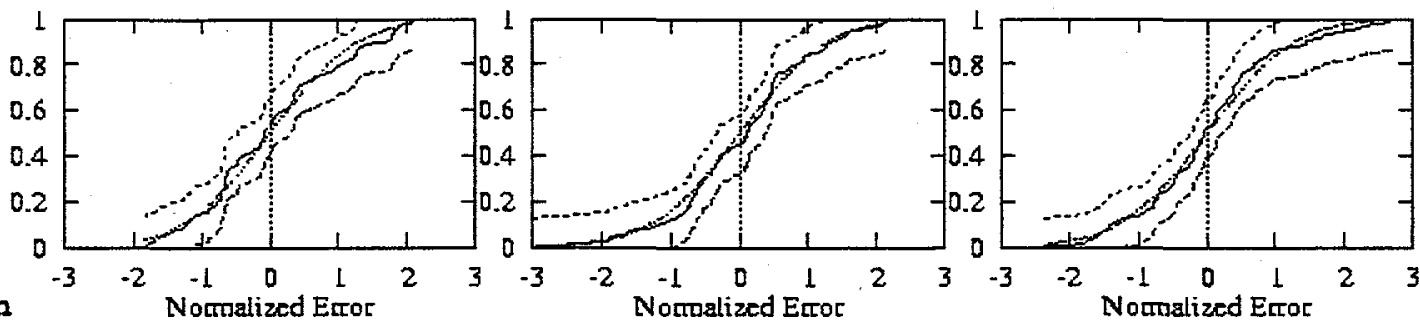

$36 \mathrm{~h}$
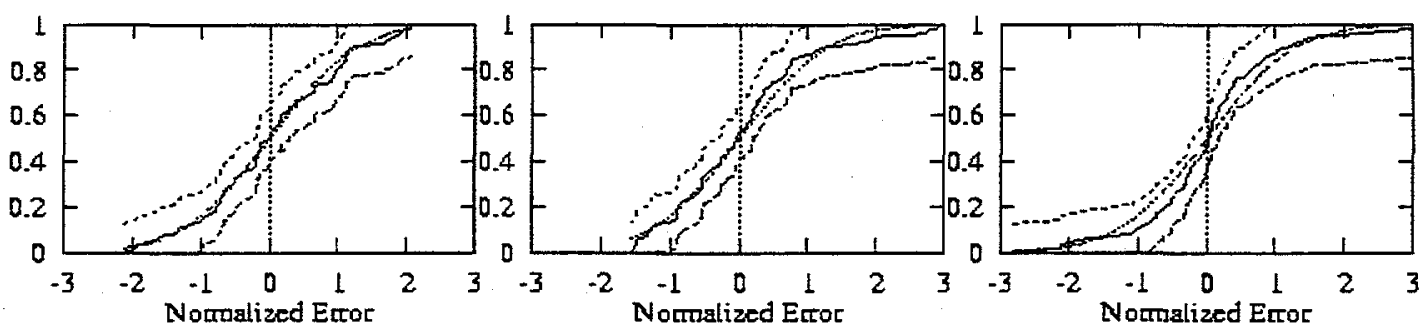

Fig. 6. $\mathrm{K}$-S statistics for satellite II.

Figure 6 illustrates K-S constructions for satellite II for along-track, cross-track, and radial prediction errors at $0,12,24$, and 36 hours. These constructions correspond to the distributions illustrated in Fig. 4. By inspection, we can see that none of the standard normal cumulative distributions intersect the curves for the $80 \%$ confidence limits. Therefore, this example provides no evidence contrary to the notion that the error distributions are normally distributed.

\subsection{RMS Errors and 95 and $99 \%$ Confidence Limits}

In many applications, decision criteria are constructed using measures of the widths of error distributions. Typical measures of distribution widths include the sample standard deviation (or rms error) and 95 and $99 \%$ confidence limits. As these quantities increase along with the width of a distribution, they also provide a convenient method for illustrating how prediction error distributions grow as a function of predict time.

To construct 95 and $99 \%$ confidence limits for empirical distributions, we sort the samples in ascending order and then find the samples, close to each tail, that are greater or less than 97.5 or $99.5 \%$ of the 
remaining samples, as appropriate. Thus, the $95 / 99 \%$ confidence limits enclose $95 / 99 \%$ of the samples, respectively. The sample standard deviation is calculated in the usual way.

Figure 7 illustrates rms errors and 95 and $99 \%$ confidence limits for along-track, cross-track, and radial error distributions for 0-, 6-, 12-, 18-, 24-, 30-, and 36-hour predict times for satellite II. Figure 7A illustrates these curves for along-track errors. Figure $7 \mathrm{C}$ illustrates these curves for radial errors. Reflecting the faster-than-linear manner in which individual error predictions grow, these curves also grow nonlinearly. Figure $7 \mathrm{~B}$ illustrates these curves for cross-track errors. Reflecting that the underlying individual predictions do not change as a function of predict time, these curves remain roughly constant as predict time increases. These behaviors are characteristic of the error distributions we have observed in our population. Appendix B contains illustrations of the growth of error distributions for all of the satellites studied.

\section{RESULTS}

\subsection{Low-Earth Orbits}

The LEO grouping contains 19 satellites. Data acquisition rates for 15 of these satellites were high, whereas the data for the other 4 were poorer with regard to both quantity and quality. The rms errors, or standard deviations, for the full set of 19 LEO satellites are displayed in Fig. 8. In these plots the subset of 15 satellites corresponds to the sets of curves clustered together toward the bottom of the plots at low rms error values. Figure 9 illustrates the radial prediction errors for this subset. We first discuss the results for the subset of 15 satellites and then the changes in prediction errors seen in the other satellites.

\subsubsection{Error Profiles and Standard Deviations}

Although the amplitudes of the errors vary from satellite to satellite, the along-track errors and the radial and cross-track errors each exhibit a number of characteristic patterns. First, cross-track errors for each satellite remain fairly constant over the entire 36-hour predict interval. The rms cross-track errors are small, ranging from 12 to $33 \mathrm{~m}$. Second, the radial errors behave in a more complex manner. The 15 LEO satellites fall into two groups with respect to the radial errors. In one group, there is a smooth, fairly linear growth in the errors. In the second group, the errors start out small and then increase more rapidly over the later portions of the predict interval. The magnitudes of the errors are intermediate between the crosstrack and along-track errors. The rms radial errors at time 0 hours fall within the range of 12 to $35 \mathrm{~m}$. At 36 hours they increase from 10 to $40 \mathrm{~m}$ for the first group and from 10 to $65 \mathrm{~m}$ for the second group.

Third, the along-track errors for each satellite are by far the largest of the three components. At time 0 hours, the standard deviations span the range from 72 to $188 \mathrm{~m}$. The rate of error growth increases as a function of the predict interval. At 36 hours, the rms along-track errors vary from 339 to $2157 \mathrm{~m}$. As can be seen in Fig. 2, the along-track errors differ from the others. While the cross-track and radial errors oscillate back and forth across the $\mathrm{x}$-axis, the along-track errors move off of the $\mathrm{x}$-axis rather monotonically in either the positive or the negative direction. That is, in each 36-hour predict interval, the predictions do not oscillate about zero error but instead either jump progressively ahead of the predictions or fall further behind the predictions. This behavior is qualitatively consistent with that predicted by Carlton-Wippern in his Langevin analysis [10]. 


\section{A}
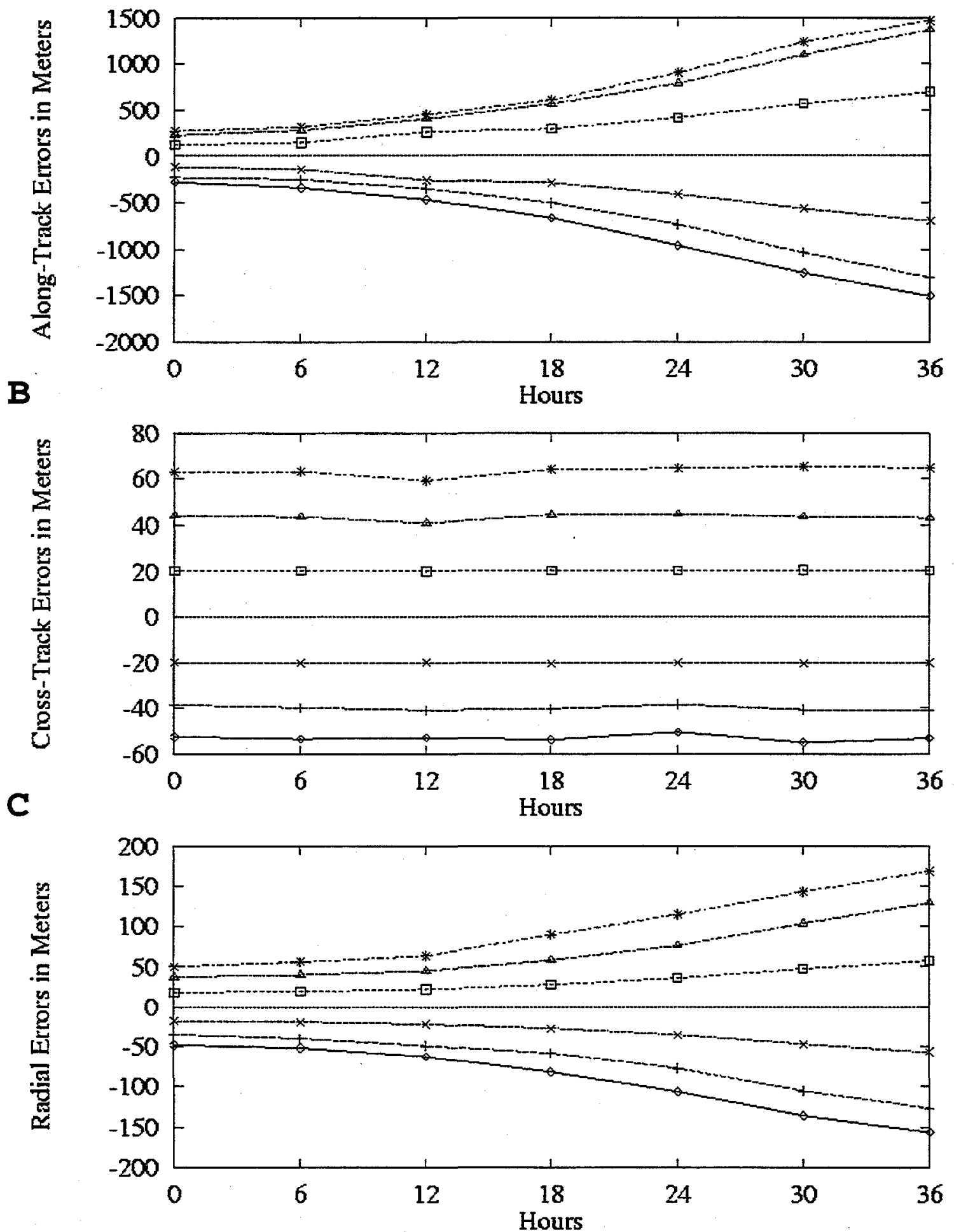

Fig. 7. Error growth as a function of predict time, satellite II. Illustrated are 99 and $95 \%$ confidence limits as well as $+/$-rms error. (A) Along-track errors. (B) Cross-track errors. (C) Radial errors. 
A
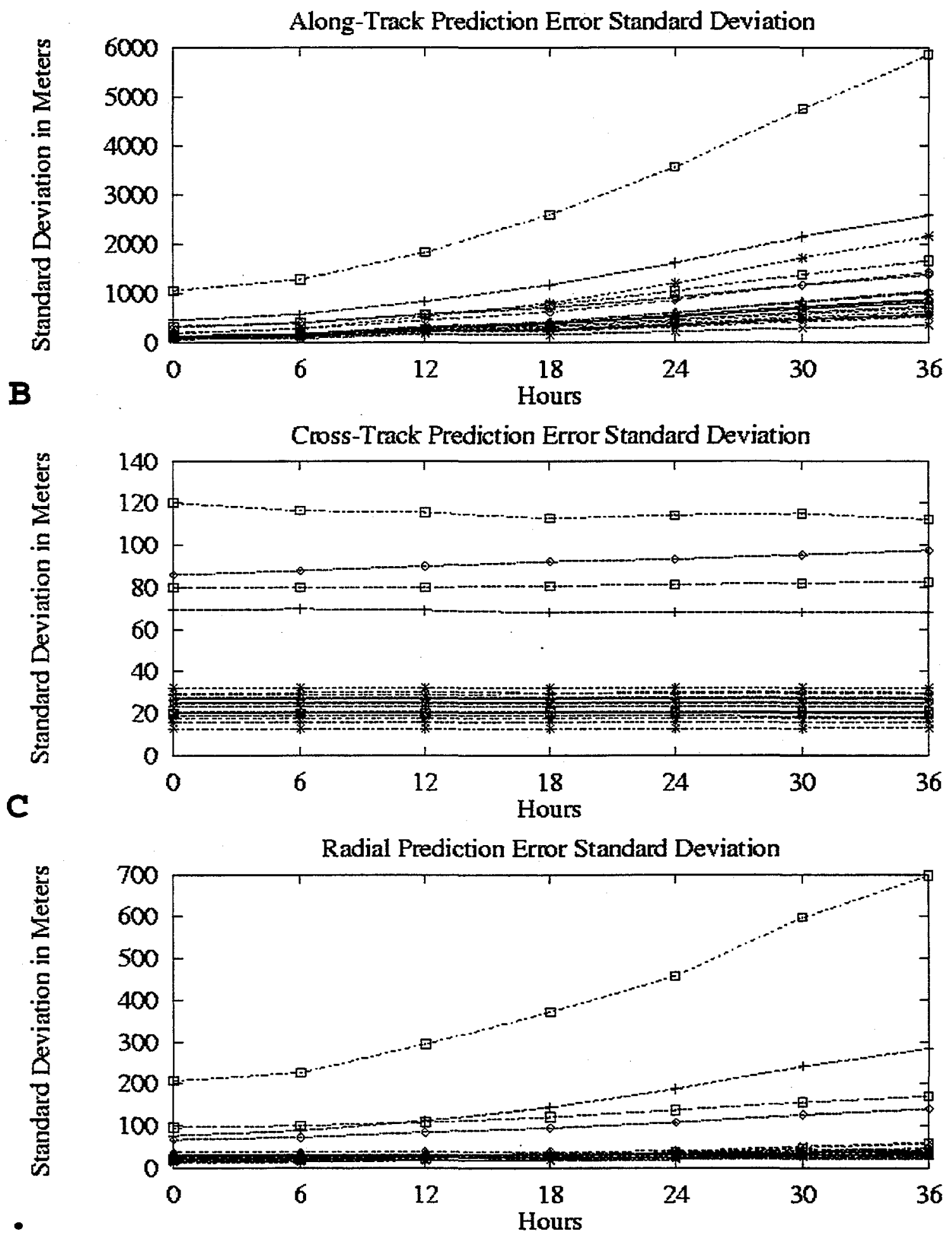

Fig. 8. Rms prediction errors for LEO satellites. (A) Along-track errors. (B) Cross-track errors. (C) Radial errors. 
Differences in the temporal behavior between the along-track errors and the radial and cross-track errors were explored analytically by Carlton-Wippern [10]. In his analysis, the Langevin equation was used together with first-order perturbation theory to develop a set of stochastic differential equations that govern the theoretical growth of satellite position error variances for the along-track and radial and crosstrack components of motion. Within the assumptions of his model he showed that the radial and crosstrack errors were bounded and do not increase monotonically in time in the same manner as the alongtrack variance. The along-track errors undergo a "random walk" and thus tend to increase in time in either the positive or negative direction. They start off quadratic, turn linear, and then increase without bound. Other errors start off linearly and then level off at some bounded value. Carlton-Wippern noted that unmodeled drag forces dramatically increase the diffusion in the along-track direction.

The rms position errors for satellites in LEO are dominated by the along-track errors. This behavior is qualitatively consistent with the cross-track, radial, and along-track errors for LACE (apogee: $467 \mathrm{~km}$; perigee: $450 \mathrm{~km}$ ) presented by Vallado [11]. The predominance of the along-track errors for the LEO satellites is a consequence of atmospheric drag. We elaborate on and refine this finding throughout the remainder of Sec. 3.

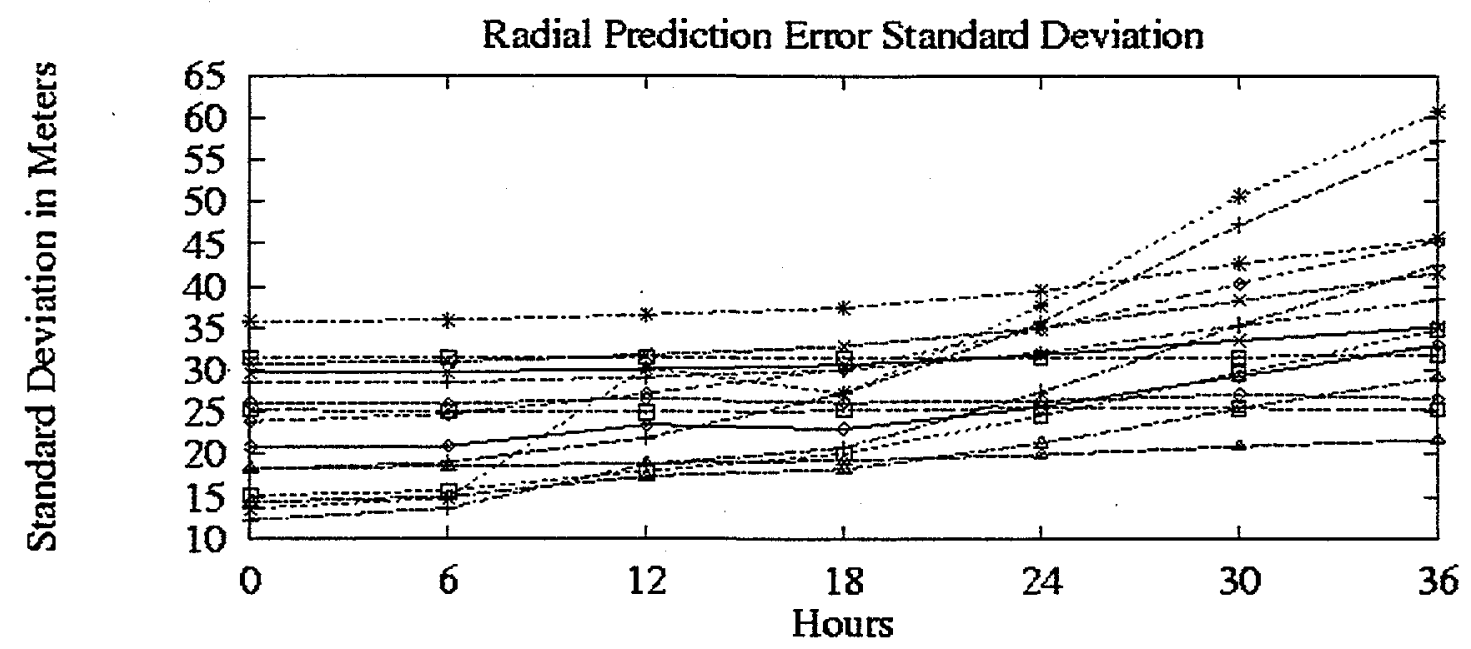

Fig. 9. Radial prediction errors for 15 of the 19 LEO satellites. The $y$-axis scale has been expanded from the previous figure to illustrate additional detail.

\subsubsection{Statistical Properties of Error Densities}

One possible implication of the behavior of the along-track, radial, and cross-track errors is that the underlying probability density functions may be complex. In particular, the possibility has been raised by Carlton-Wippern [12] that the probability densities may not be concentrated about their mean rms predicted position values. As a result of the behavior of the along-track errors, the characterization of the error density solely in terms of the rms error may lead to misleading conclusions when used for making either probabilistic or deterministic collision estimates. The rotation of a bifurcated error density function to accommodate for closest approach orientation might generate a folded surface, and one must perform the requisite integration in a Lebesgue sense to correctly estimate the probability of collision [12].

This not unreasonable expectation was examined for the 15 LEO satellites and was found not to occur. To understand why it does not occur, we must discuss the significance of the error densities as represented by the error histograms. First, we recall that the error histograms were generated for each space object by combining the errors at representative time steps for the collection of sampling intervals. This procedure allowed us to generate a sequence of histograms representing the time evolution of the errors for each space object. This was done separately for the cross-track, radial, and along-track errors. 
Widths: If we inspect the histograms, we observe that the widths of the cross-track histograms tend to remain fairly constant over time, while those for the radial and along-track histograms increase over the $0-, 12-, 24-$, and 36-hour time intervals. The temporal evolution of the widths is fully consistent with our expectations based on the raw data presented in the error plots. We observe the constancy of the crosstrack errors and the increasing character of the radial and along-track errors.

Mean values: The histograms represent the ensemble of errors for each selected satellite. These histograms, including those for the along-track errors, are peaked about their mean values. The mean errors in the radial and cross-track directions are all nearly zero, while those for the along-track errors are nonzero, but small. Although the individual errors in predicted position in the along-track or any other direction in any given predict interval fall away from zero as predicted by the Langevin analysis, the ensembles of errors do not.

The overall shape of the error histograms is what might be expected from atmospheric effects for the following reasons. The overall scale of each histogram is established by the span of the errors for that satellite. If we return to the error plots, we see for each satellite a large number of small errors that correspond to situations where the atmospheric perturbations do not appreciably change between the fit interval and the predict interval. In these cases the drag-related errors are small. In a few instances there are appreciable changes in atmospheric perturbations between the fit and predict time intervals. In these cases the prediction errors are large. Thus, the amplitude of the errors for any given predict interval may be regarded as an atmosphere-related random variable that plays a key role in establishing the form of the error densities.

Kolmogorov-Smirnov Statistic: We conducted a detailed series of K-S tests to characterize further the statistical properties of the error densities. As pointed out in Sect. 2, the K-S tests first permitted us to establish confidence bands for the distribution functions constructed from the data represented in the error histograms. They then allowed us to test the hypothesis that a random sample came from a population with a specified distribution function. In particular, they permitted us to test the hypothesis that the errors in the cross-track, radial, and along-track directions are Gaussian distributed. This assumption is made in the NASA collision avoidance procedure and for that reason is of interest. These tests were carried out separately for each satellite for the combined error distributions for an ensemble of 15 LEO satellites and for the full sample of 19 satellites. In forming the combined sample, we scaled the errors for each satellite by their rms deviation after shifting for the mean. The resulting sample contained more than 1000 events for each time slice. The results of the K-S test for the combined sample are shown in Fig. 10. We see in these plots that the curves representing Gaussian distributions remain within the confidence bands. Similar results were obtained when we applied the K-S statistic to the smaller samples for individual satellites. Thus, the assumption of a set of Gaussian-distributed random variables is not inconsistent with the data.

\subsubsection{Regression Analysis}

To further our understanding of the errors, we correlated the magnitude and rate of change of the alongtrack, radial, and cross-track error distributions to orbit parameters such as semimajor axis, apogee, perigee, eccentricity, and inclination and their rates of change. For each satellite, we used least-squares methods for determining the average and the average rate of change in orbit parameters during the study intervals. We then combined the estimate across the population of satellites to determine correlation between errors/error growth and orbit parameters. 
A
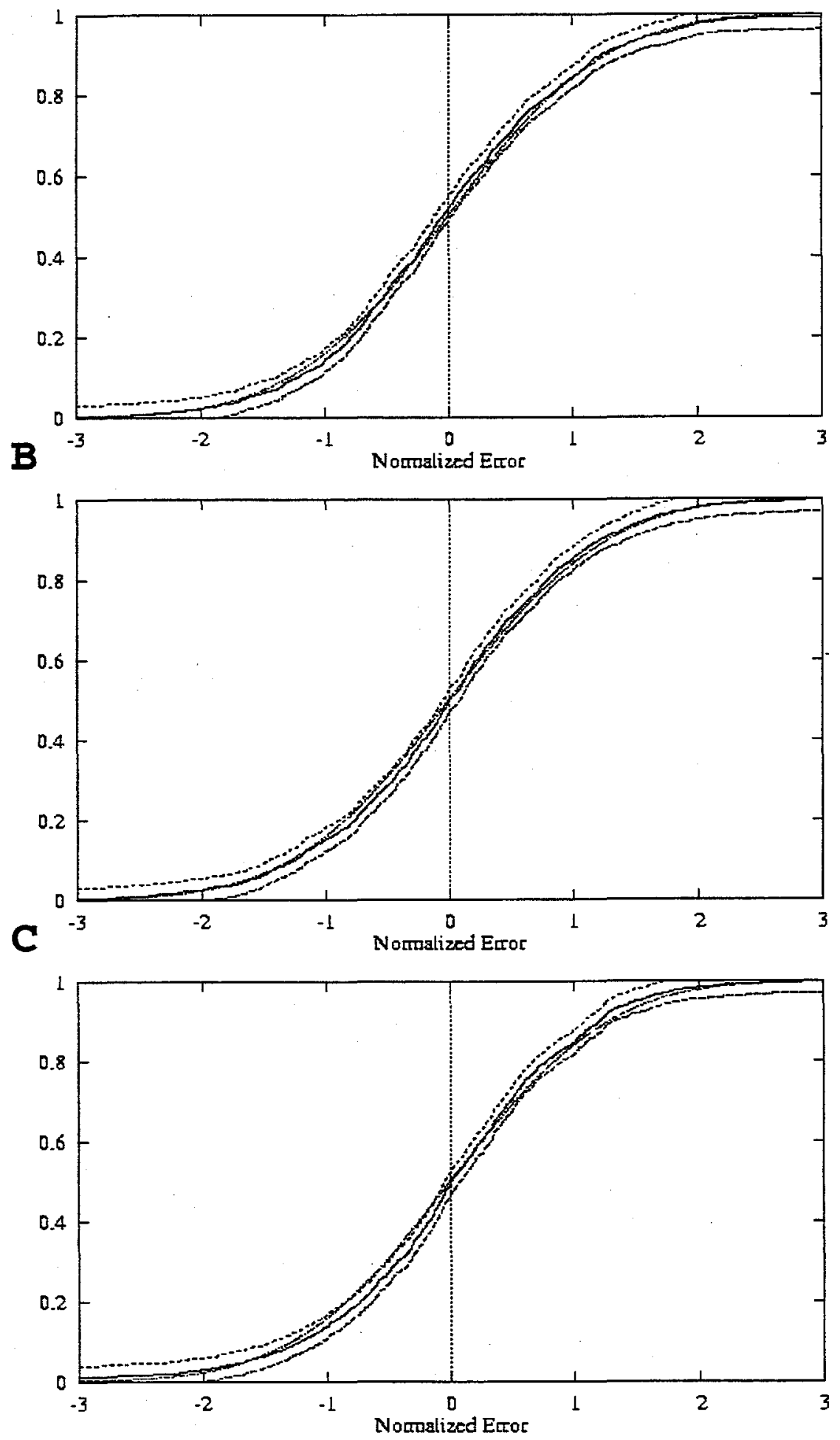

Fig. 10. K-S statistics for combined sample of all LEO satellites at 36-hour predict time. (A) Along-track errors. (B) Cross-track errors. (C) Radial errors. 
A

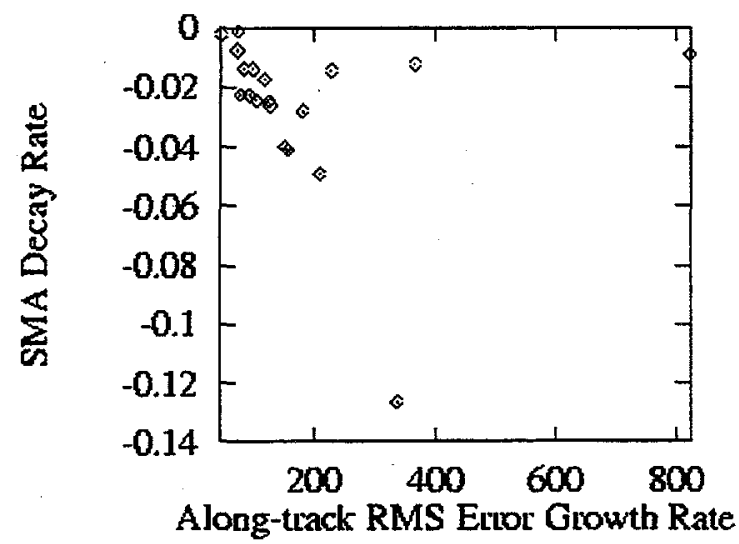

C

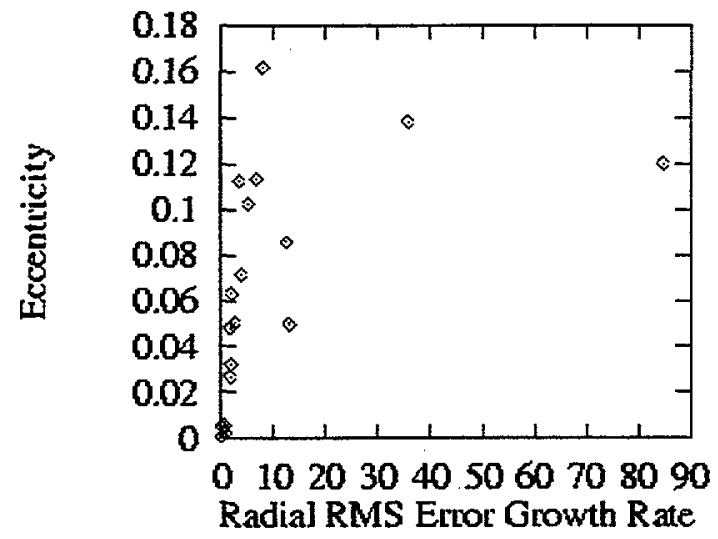

B

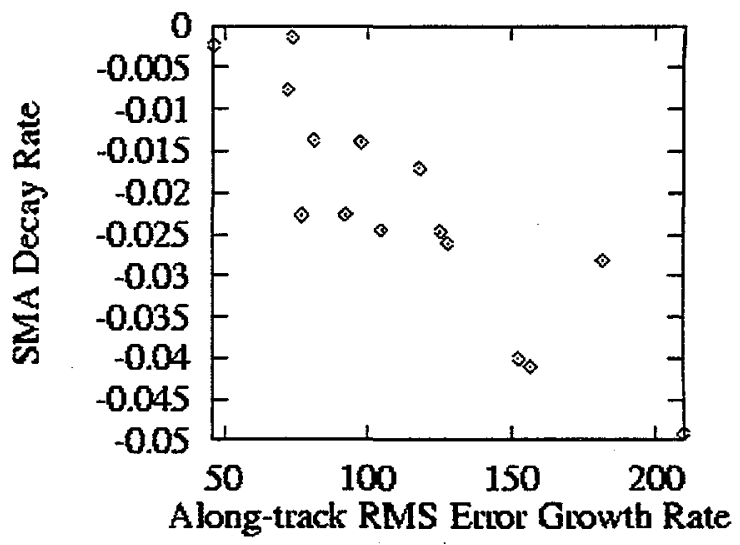

D

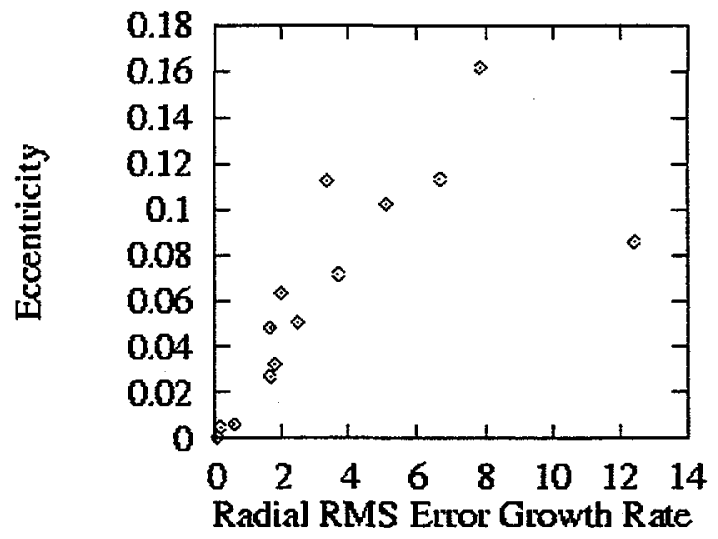

Fig. 11. Correlation of semimajor axis decay rate and along-track error growth rate. Satellites YY, 11 , 22 , and 33 are discarded from $B$ and $D$.

The results of the regression analysis are presented in Fig. 11. Displayed in this figure are the two most significant correlations for the subsample of 15 satellites and for the full population of LEO satellites. In examining this figure, we see that the along-track error growth rate is correlated with the decay rate of the semimajor axis. The correlation is strongest for the subpopulation of $15 \mathrm{LEO}$ satellites and is somewhat weakened by the addition of the other satellites composing the full sample. The decay of the semimajor axis is a consequence of the nonconservative character of atmospheric drag forces. This correlation serves to link a significant portion of the prediction errors to the presence of unmodeled drag forces, that is, to inadequacies in the drag model and its parameters. The other two parts of Fig. 11 illustrate a strong correlation between the radial error growth rate and the orbit mean eccentricity. This correlation will be discussed in Sect. 3.3, where we present the HEO data.

Degradation in the data quality and quantity can contribute substantially to prediction errors, even in a strongly drag-perturbed LEO regime. If we refer back to the plots of the standard deviations along the various axes of motion, we observe significant increases in the magnitudes of the errors for those satellites not included in the subsample. This is reflected in the worsened correlation between the alongtrack error growth rate and the decay rate of the semimajor axis seen in Fig. 11. It is also observable in the error profiles. The satellites with poor data possess error profiles that appear jagged compared with the far smoother profiles for satellites having high-quality data. We elaborate on this in Sects. 3.2.1 and 3.2.2. 


\subsection{Near-Circular Orbits}

Our second group contains satellites in NCOs at mean altitudes from 700 to $1500 \mathrm{~km}$. Of the 29 satellites, 6 are in this group. Although their orbits are just beyond the LEO zone of prime interest with respect to space shuttle and ISS operations, this regime is still important. The NCO data are of interest not only because any HEO satellites must continually pass back and forth through the NCO altitude zone but also for the insights these data provide regarding the physical origins of ephemeris prediction errors.

\subsubsection{Error Profiles and Standard Deviations}

We succeeded in generating complete data records for five of the satellites in the NCO category and in constructing a partial record for the sixth. The along-track, radial, and cross-track error profiles for all six records are presented in Appendix A. The corresponding time evolution plots of the rms errors from 0 to 36 hours are shown in Fig. 12. As was the case in the LEO regime, the along-track errors are larger than either the radial or cross-track errors. The growth in the radial and across-track errors is modest throughout the 36-hour predict intervals, while the errors in the along-track direction increase by factors from 2 to 5 .

The magnitudes of the errors for the NCO satellites are remarkably similar to those of the 15 LEO satellites. Both classes of satellites have 36-hour prediction errors that vary from 200 to $300 \mathrm{~m}$ at the low end to just over $2 \mathrm{~km}$ at the high end. If we restrict our attention to atmospheric effects, we might expect the errors for the NCO satellites to be smaller than those for the LEO satellites. There is indeed evidence for a reduction of the errors as the mean altitude increases. Satellite AA has the smallest prediction errors and is in the highest orbit of the group. Satellite FF is in the next highest orbit, and its errors are mostly smaller than those of the other NCO satellites. However, the overall decline in importance of atmospheric effects for the NCO satellites is matched by an increase in uncertainties associated with the data themselves.

\subsubsection{Quality and Quantity of Data}

In the NCO regime we encounter degradation in data quality and quantity. The decline in the quantity of data, or frequency of observation per fit interval, is apparent in the observation statistics given in Table 2. Evidence for a degradation in quality and a connection to ephemeris prediction can be found by comparing the scatter in observed ranges, azimuths, and elevations associated with the smoothed-fitted values for satellites in the LEO and NCO regimes. We made comparisons of this type for a pair of satellites, TT and EE. The former is a LEO satellite, and the latter is an NCO satellite. The overall ephemeris errors for these two satellites are comparable, and this pair was chosen for that reason. The main result of the comparisons is that the scatter (i.e. the differences between data and fitted curves) is far greater for the NCO satellite than for the LEO satellite. The scatter in azimuth measurements about the fitted values is more than a factor of 2 greater for the NCO satellite than for the LEO satellite. This is the most pronounced of the increases. The scatter in range increases by about $25 \%$, while larger increases in scatter are observed for elevation.

Another aspect of the dependence of prediction errors on data quality and quantity is illustrated by error profiles. If we examine the error profiles, we observe the appearance in each satellite profile of one or more predict intervals where the errors are more than five standard deviations above the mean. With few exceptions, most notably satellites WW and UU, error spikes do not occur in the LEO data set. The LEO satellite UU has the lowest data rate of all and not coincidentally has an error spike and did not provide a complete record. The data acquisition rates for satellite WW are comparable to those for the NCO satellites. Lastly, if we compare standard deviations for the full LEO ensemble to the NCO satellite set, we see that the overall errors for the NCO satellites are indeed reduced. 
A
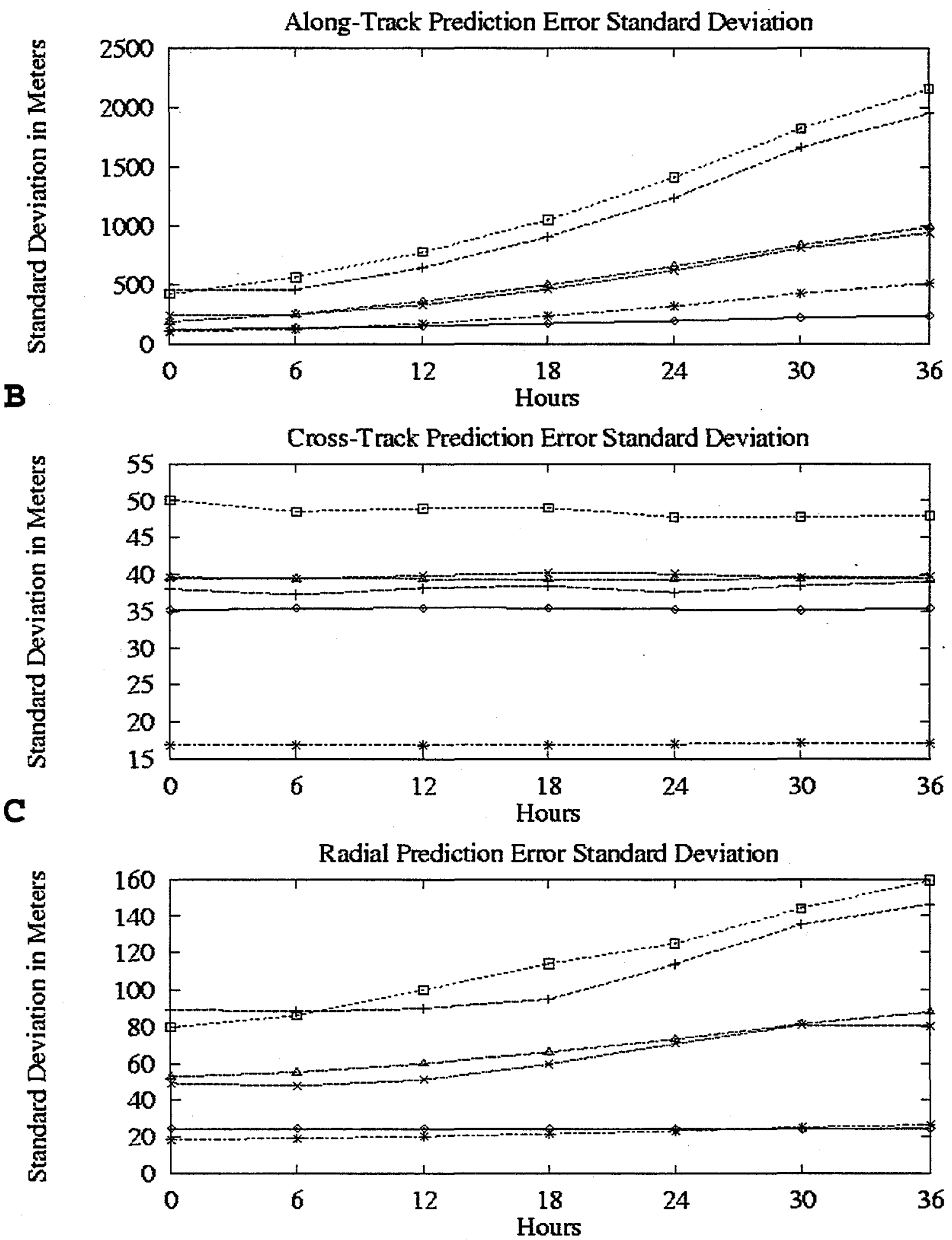

Fig. 12. RMS errors for satellites in high altitude, NCOs. (A) Along-track errors. (B) Cross-track errors. (C) Radial errors. 
A
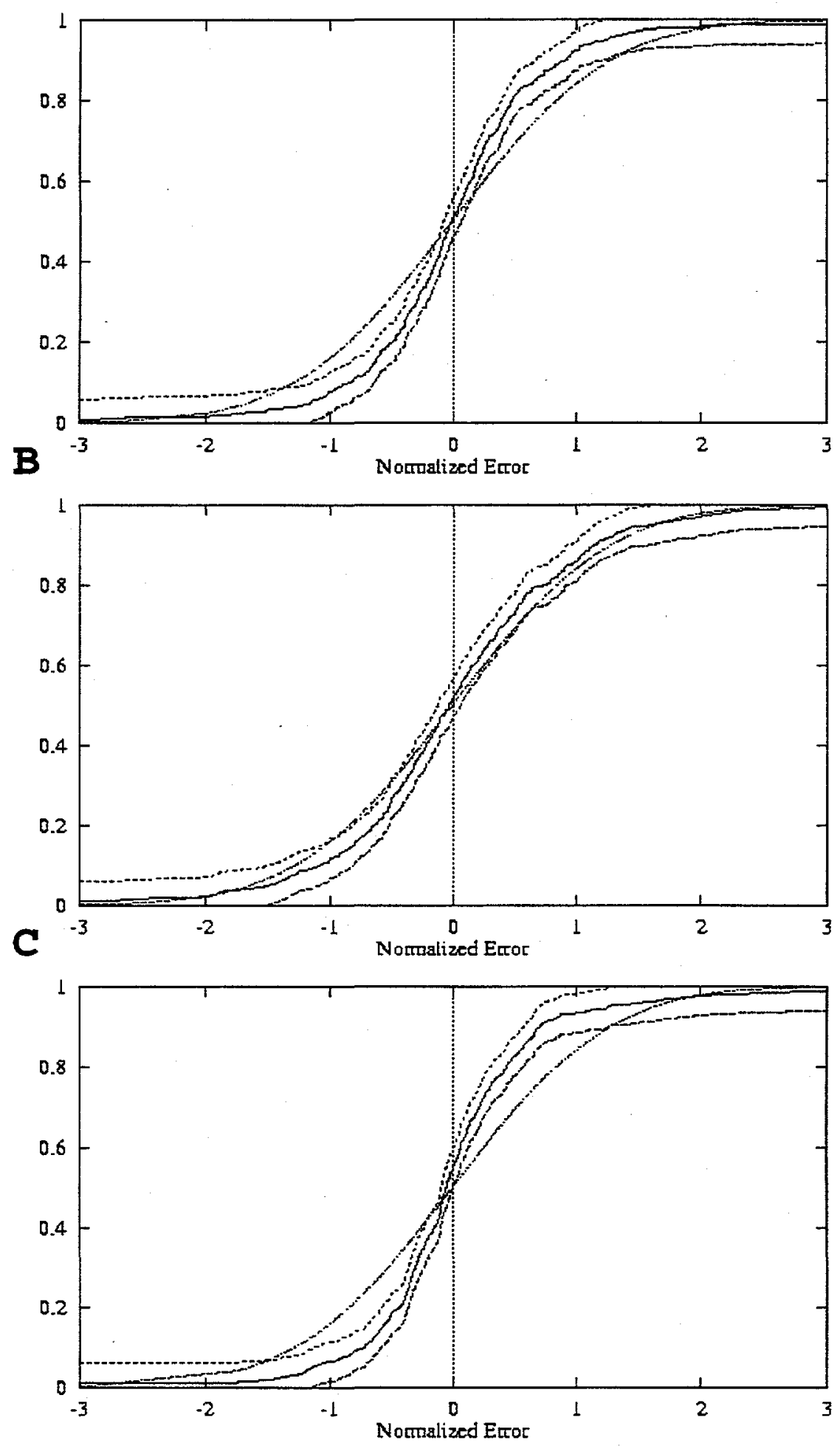

Fig. 13. K-S statistic for the combined sample of NCO satellites. (A) Along-track errors. (B) Cross-track errors. (C) Radial errors. Because of the presence of spikes in the predictions, errors in the along-track and radial directions are not normally distributed. 


\subsubsection{Statistical Properties of Error Densities}

The statistical properties of the NCO satellites are interesting. We performed a $\mathrm{K}-\mathrm{S}$ analysis of the error densities for the NCO population in the same manner as for the LEO satellites. As was the case for the LEO satellites, we used a Gaussian distribution as the null hypothesis. The results for the full population of the along-track, radial, and cross-track error densities are presented in Fig. 13. In contrast to our findings for the LEO satellites, the NCO satellites dramatically fail the K-S test. The failure can be seen easily in both the radial and along-track K-S plots, it is also present in the cross-track plot.

One way of interpreting this result is to note that the spikes in the error profiles resemble that of shot noise. That is, we are in a regime where there are regions of shot like noise interspersed within larger relatively quiescent regions. In the NCO regime, the presence of error spikes drives the data far away from the limits expected from a Gaussian distribution.

\subsection{High Eccentricity Orbits}

We have grouped together results of analyses of four satellites characterized by HEOs. The eccentricities for these satellites vary from 0.22 to 0.52 . In each orbit cycle the satellites dip down into the thermosphere to altitudes of 300 to $400 \mathrm{~km}$ at perigee and then move out to altitudes from 4000 to almost $15000 \mathrm{~km}$ at apogee. The creation of a category for these satellites is motivated by several factors. First, these satellites have trajectories that dip down far into the LEO regime. Consequently, these orbits are of special importance with respect to space shuttle and ISS operations. Second, although there is some advantage to including these satellites in the LEO group to broaden the parameter range for the regression analysis, the data for HEOs provide us with new insights and pose special challenges.

\subsubsection{Error Profiles and Standard Deviations}

Displayed in Appendix A are the error profiles for the four HEO satellites. As can be seen in these plots, two of the satellites, DD and $\mathrm{HH}$, yielded a full set of 72 predict intervals. The series of SP calculations for the other two satellites, RR and ZZ, were terminated by GTDS. Before the termination, however, the calculations provided partial error profiles for the time intervals that the satellites were tracked.

The first and most obvious observation is that the errors in all directions of motion are far greater for the HEO satellites than for the LEO satellites in the corresponding directions. As was the case for the LEO satellites, the along-track errors exceed the cross-track and radial errors. The radial errors are intermediate between the along-track and cross-track errors. However, they are no longer an order of magnitude smaller than the along-track errors. The relative magnitudes of the errors along the different axes of motion are consistent with a decline in dominance by drag.

The standard deviations in the along-track, radial, and cross-track directions for each of the HEO satellites are shown in Fig. 14. As was the case for the other regimes, the cross-track errors are smaller than the others and remain fairly constant throughout the 36-hour predict interval. However, these errors can now reach $1 \mathrm{~km}$ at 36 hours. The along-track and radial errors are large and grow in time. The alongtrack errors vary from a few hundred meters to $4 \mathrm{~km}$ at time 0 hours and from 3 to $9 \mathrm{~km}$ at 36 hours. The radial rms errors range up to $700 \mathrm{~m}$ at time 0 hours and can exceed $2.5 \mathrm{~km}$ at 36 hours. One consequence of the simultaneous occurrence of large along-track and radial errors is that the predicted satellite orbits "wobble" back and forth from one predict interval to the next. 
A

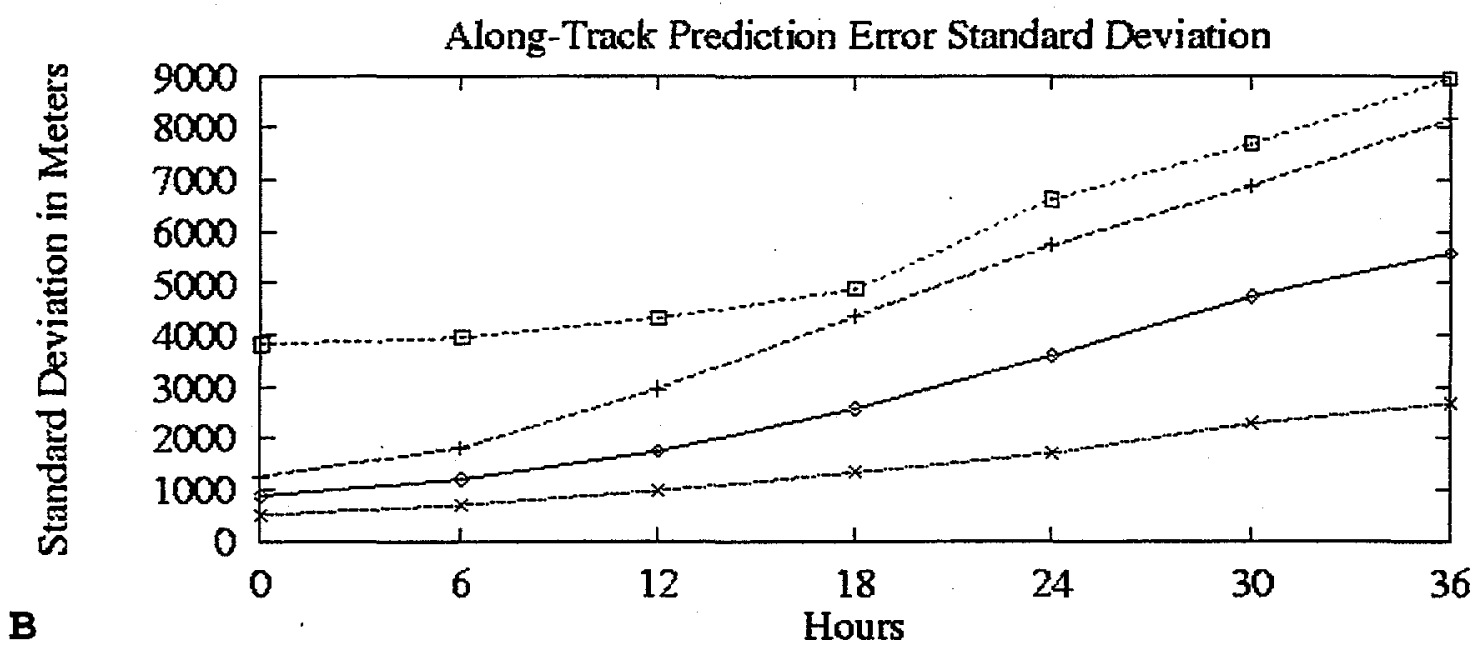

$\mathbf{B}$
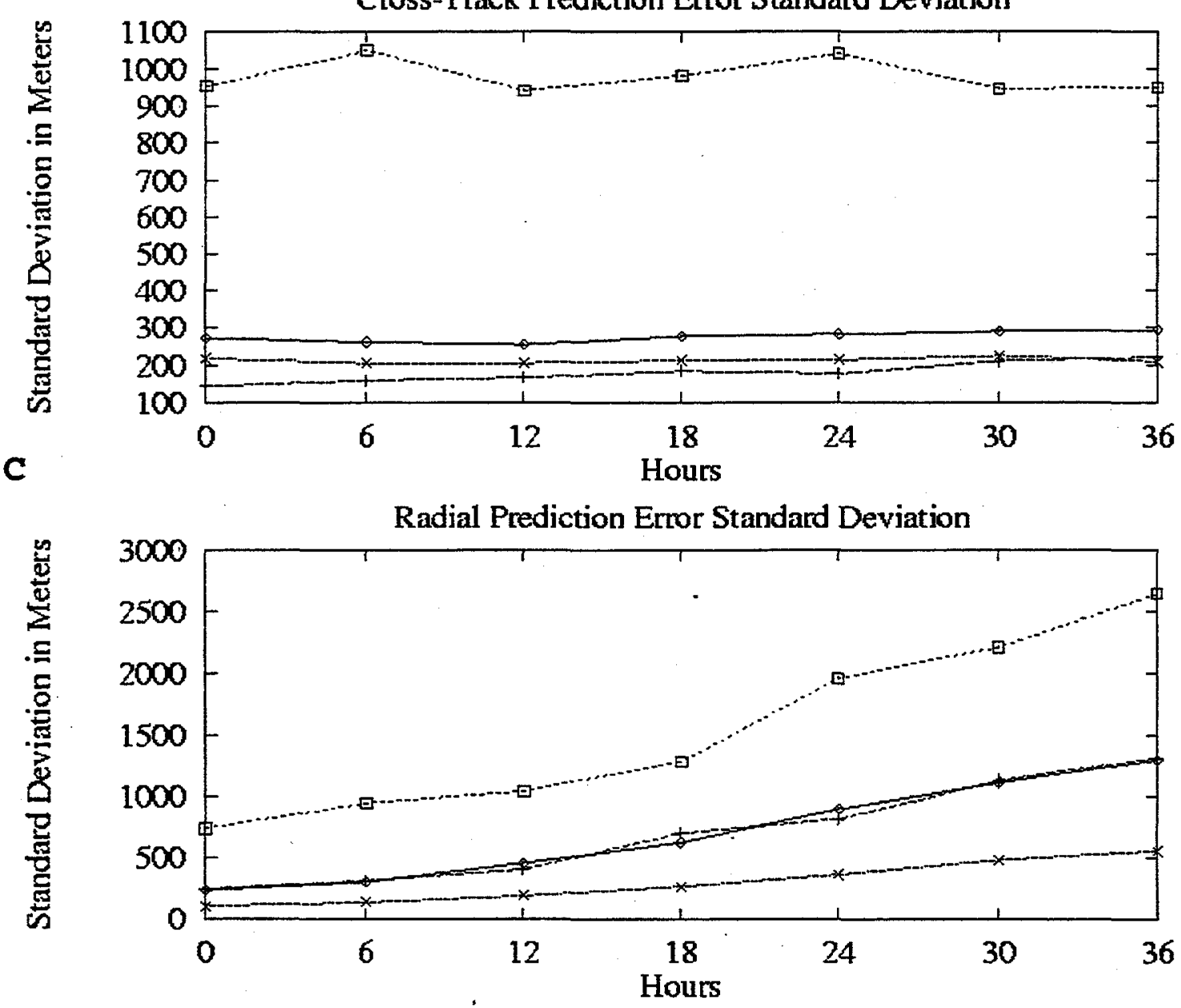

Fig. 14. RMS errors for satellites with HEOs. (A) Along-track errors. (B) Cross-track errors. (C) Radial errors. 


\subsubsection{HEO Observational Data}

Listed in column 2 of Table 2 are the minimum numbers of 36-hour DC runs. There are 25 complete records corresponding to 66 or more runs and 4 partial records. The next four columns contain the run statistics, namely, the minimum number of observations in a 36-hour DC period, the maximum number of observations, the average number of observations over the record, and the rms deviation in the number of observations per 36-hour DC interval.

The numbers of observations vary considerably both from satellite to satellite and from interval to interval for a particular satellite. The most consistent finding is that the numbers of observations for the HEO satellites are far lower than for the LEO satellites. The average numbers of observations for the HEO satellites vary in a narrow range from 66 obs/interval for satellite DD to 105 obs/interval for satellite $\mathrm{HH}$. These numbers may be compared with average numbers of obs/interval as high as 350 or more for some of the LEO satellites. The decline in observational density is even more dramatic if we consider the HEO minimum numbers of observations listed in col. 2. These quantities vary from 25 to 35 obs/interval, and may be compared with values as high as 250 obs/interval for some of the LEO satellites.
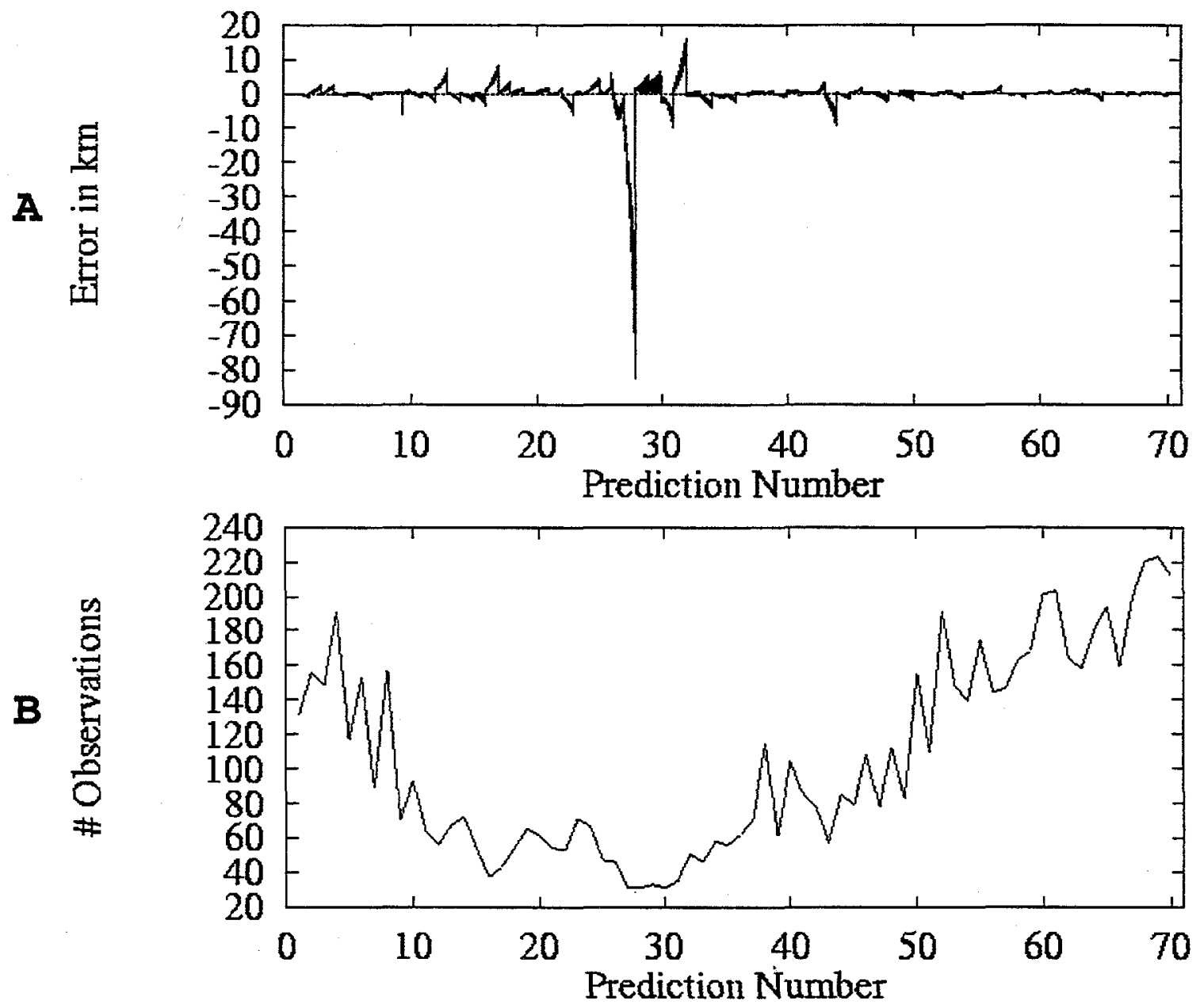

Fig. 15. Illustration of the relationship between density of observations and error magnitude. (A) Alongtrack errors. (B) Number of observations per 36 hours. The reduced density of the observations during the central portion of the run may explain both the increased error during that period and the prominent spike in the data. 
The number of observations is clearly correlated with the magnitudes of the errors, but the dependence is complex. Figure 15 illustrates a plot of the number of observations for each 36-hour interval for satellite $\mathrm{HH}$. If we compare the observation plot with the error plot, we not only see a smooth modulation but also find that the steep decline in observation frequency is correlated with the appearance of a large error spike. Other less well developed spikes occur in the low data rate regime, and as the observational frequency increases, the magnitudes of the errors decline.

\subsubsection{Statistical Properties of the Error Densities}

We performed a K-S analysis of the error densities for the HEO population in the same manner as for the LEO and NCO satellites. As was the case for the other satellite groups, we used a Gaussian distribution as the null hypothesis. The results for the full population of the along-track, radial, and crosstrack error densities are presented in Fig. 16. As was the case for the NCO satellites, the HEO satellites dramatically fail the K-S test. Again, the failure can be seen easily in the radial and along-track K-S plots and is also present, although less apparent, in the cross-track plots. These findings reinforce the interpretations presented in Sect. 3.2.3 for the NCO satellite data.

\section{CONCLUSIONS}

\subsection{Summary of findings}

We have presented detailed results of an error analysis for 29 satellites taken from a population of approximately 110 satellites. The 29 satellites analyzed are in orbits that are of interest with respect to space shuttle and ISS collision avoidance and provided us with sufficient error records for analysis. These satellites were placed into one of three groupings according to their orbit characteristics: LEO, NCO, or HEO. For each satellite we determined the error densities in the along-track, radial, and cross-track axes of motion for sequences of 0- to 36-hours prediction intervals over several months. We determined the standard deviations in the error densities, examined their statistical properties, and delineated the $95 / 99 \%$ confidence levels.

General Characteristics of the LEO Errors. The LEO satellites were grouped according to data quality and quantity into high and low categories. Although the errors vary from satellite to satellite and from one fit interval to the next we can make the following general statements about the high-quality/quantity errors. The along-track errors predominate; their rms values range from $200 \mathrm{~m}$ to more than $2 \mathrm{~km}$ at the 36 -hour predict time. These errors increase monotonically throughout each 36 -hour predict interval. The predicted positions in the along-track direction progressively either leap ahead of or fall behind the actual positions. The radial and cross-track errors are smaller than the along-track errors by roughly an order of magnitude. Unlike the along-track errors, the radial and cross-track errors oscillate back and forth about their near-zero mean values. The cross-track errors remain nearly constant throughout the 36-hour predict intervals, while the along-track errors and amplitudes of the radial errors increase. In LEO cases where data quality and quantity were low, sensor-data-related uncertainties contribute substantially to the error densities.

Unmodeled Drag Forces. Systematic errors arising from unmodeled drag forces are present in the error profiles for all LEO satellites. We performed a regression analysis of the errors for the LEO satellites. This analysis correlated the magnitudes of the errors and their rates of growth with the physical characteristics of the orbits and produced an error model as its outcome. The regression analysis of the high-quality/quantity data revealed a strong correlation between the along-track error growth and the

decay rate of the satellite semimajor axis. Because the decay of the semimajor axis is a consequence of the nonconservative drag forces, this correlation serves to link the systematic along-track errors to inadequacies in the atmospheric drag model and its parameters. The correlation was weakened somewhat by the inclusion of the low-quality/quantity LEO data. 
A
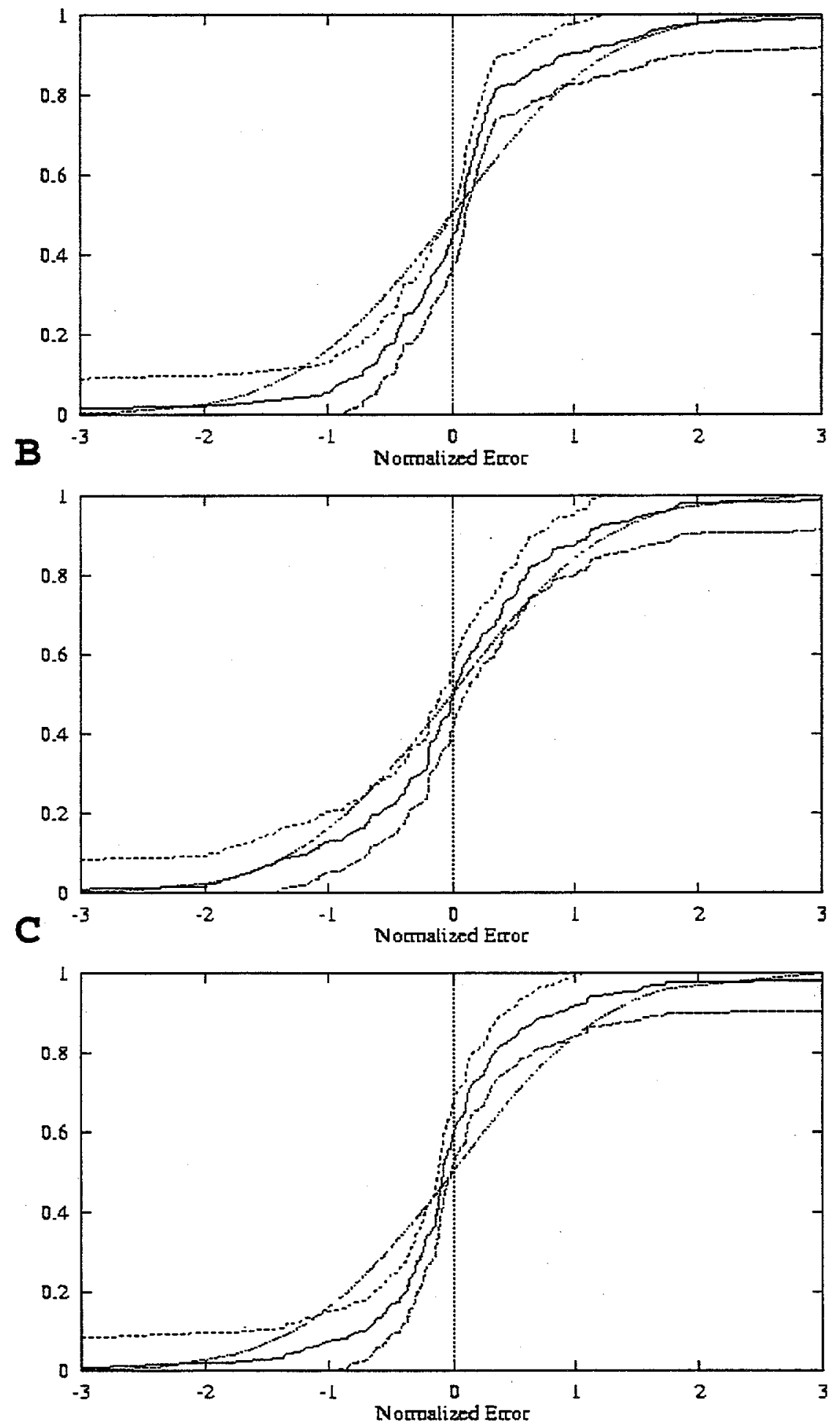

Fig. 16. $K$-S statistic for the combined sample of HEO satellites. (A) Along-track errors. (B) Cross-track errors. (C) Radial errors. Because of the presence of spikes in the predictions, errors in the along-track and radial directions are not normally distributed. 
HEO Errors and Orbit Wobble: Progressively larger ephemeris prediction errors along all axes of motion are encountered as the satellite orbits become more eccentric. In the HEO regime, the rms errors in the along-track direction vary from 3 to $9 \mathrm{~km}$ at 36 hours. The comparable errors in the radial direction range from $500 \mathrm{~m}$ to almost $3 \mathrm{~km}$ while those in the cross-track direction are about a factor of three smaller. In the LEO regime, we find that the radial error growth rate is correlated with the mean eccentricity of the orbit. That is, the greater the eccentricity the larger the rate in increase in radial errors throughtout the 36-hour predict interval. In the LEO regime, the eccentricities are small, less than 0.2 , and radial errors remain small compared with along-track errors. In the HEO regime, where eccentricities are considerably larger, we observe radial errors on the order of several kilometers. There is a pronounced orbit wobble in this regime.

NCO Errors, Quality/Quantity Effects, and Error Spikes. In the NCO regime, the decline in prediction errors associated with a decreasing importance of unmodeled drag forces is offset by increasing uncertainties in the sensor data. The rms errors in the along-track direction vary from about $200 \mathrm{~m}$ to slightly over $2 \mathrm{~km}$, and we find that the NCO errors are comparable to those in the LEO regime. Both the quantity and quality of the sensor data contribute to the precision of the orbit determination in all three regimes. As the number of observations per fit interval decline, the magnitudes of the prediction errors increase.

In situations where the quality and quantity of data are low, spikes appear. These spikes, defined as errors of more than five standard deviations, are most pronounced in the NCO data but appear in other regimes whenever the data are degraded. They can be seen most easily in the error profiles for the alongtrack axis of motion and are also prominent in the error profiles for the radial axis. These data spikes cause large increases in the $95 / 99 \%$ confidence limits, which then become much greater than the rms error values.

Statistical Properties of the Error Densities. In any predict interval, the calculated along-track positions either leap ahead of or fall behind the true positions in a manner qualitatively consistent with the results of a Langevin analysis performed using first-order perturbation theory [10]. The magnitudes of these errors, and the amplitudes of the radial and cross-track errors, are modulated in a random way by stochastic atmospheric effects and by random variations in the quantity of observational data. In the LEO regime, we find that the errors become Gaussian. This statistical property of the errors was established through K-S tests performed for individual satellites, for the joint sample of 15 LEO satellites, and for the full ensemble of 19 satellites. In the NCO and HEO regimes, the ensembles of errors dramatically fail the K-S test for Gaussian behavior.

Finite Beam Width and Surface Effects. In examining the progressively larger errors encountered in treating HEOs we are reminded that the conversion of sequences of returns from range sensors such as radar and sonar into target positions is subject to a variety of systematic errors arising from finite beam width effects [13]. These errors influence the accuracy of satellite orbit determinations, while the reduced numbers of observations leading to sparser data for the HEO satellites affect their precision. As is the case for sonar beam width errors, methods can be developed to reduce these errors. The finite beam width and other shortcomings in the treatment of tracks, along with the decline in quality and quantity of data and atmospheric drag, all contribute to the observed HEO wobble.

Returns from range sensors such as radar and sonar are affected in several different ways by surface properties of the objects being probed. Both the precision and accuracy of the ephemeris predictions can be influenced by interactions between beam and target surfaces. One example of a surface-property effect is degradation in the quality of the data for satellites having low radar cross-sections. This degradation reduces the precision of the orbits and influences the differential correction process where fits are made to data using preassigned standard deviations and biases. As is customary, assignments of standard deviations are made to radar units independent of beam-target and atmosphere-beam interactions. Mismatched relationships between assumed and observed scatter in the data can generate difficulties in 
the DC process. These effects are most pronounced in sparse data regimes. We observe in the NCO regime that azimuth and elevation data are degraded compared with data for satellites in lower orbits.

\subsection{Recommendations}

We have four sets of recommendations, all of which follow directly from the results of this study. The first set of recommendations relates to improving the atmospheric model, the second to improving treatment of the sensor data, the third to formalizing the operational ranges of SP and other models, and the fourth to development of enabling technologies to automate this formalism.

\subsubsection{Atmospheric Model}

We can take a number of actions to improve the reliability of the atmospheric model. In discussing these actions, it is useful to distinguish three classes of properties of the earth's atmosphere-static, dynamic, and stochastic [14]. The static part of the atmospheric density model treats the variation in density with altitude. The altitude dependence is modulated by an exosphere temperature that influences the amount of heat absorbed by the atmosphere. It also takes into account latitude variations, namely, the flattening of the earth, which produces a decrease in density from equator to pole. The dynamics part of the density encompasses a number of slow cyclical processes. These include the diurnal bulge associated with the earth's rotation, the 27-day solar rotation cycle, the 11-year solar activity cycle, the geomagnetic activity cycle, the semiannual variation, and the seasonal-latitude cycle. The stochastic component refers to short-term, hard-to-predict phenomena such as solar flares, geomagnetic storms, and perhaps thermosphere/exosphere winds.

The first set of actions that can be taken is to update the static model and those dynamic components that directly affect the exosphere temperature. A number of atmospheric models have been developed during the past few years. Of these, the Jacchia 71 and MSIS 90 [15] models are perhaps the best known [16]. Another well-known model is DTM94 [17]. The Jacchia 71 model is widely regarded as the most complete of the atmospheric models and was used by us in the SP calculations. In spite of its completeness, there are a number of shortcomings in the Jacchia 71 model of atmospheric density. Therefore, we recommend that an updated static model be constructed and incorporated into the SP model.

Two specific actions are recommended. First, we should take advantage of the work done in developing MSIS90 and other models based on recent higher-resolution data and incorporate those results where appropriate. Second, and most important, Bayesian techniques should be used to further refine the model. In this type of approach, one determines the static densities that minimize the error densities using the procedures and measures developed in the present study. The hybrid a priori atmospheric density model is updated by iteration using high-quality and -quantity satellite data. The error densities serve as a cost or risk function whose minimization yields a maximum a posteriori (MAP) estimate of the optimal static atmospheric model.

The satellite data are sparse in relation to a goal of constructing an atmospheric model that spans all latitudes, longitudes, and elevations to $2000 \mathrm{~km}$ in a smooth continuous manner. Inverse problems of this type are encountered in many disciplines. One MAP approach that has been used successfully by many researchers is to model the desired posterior density as a Markov random field [18]. One then uses any of a variety of updating schemes to construct a dense MAP estimate. This approach can be extended to include changes in atmosphere density caused by solar radiation and geomagnetic activity parameterized by the $10.7 \mathrm{~cm}$ solar radiation flux $(\mathrm{F} 10.7)$ and the daily planetary amplitude $A_{p}$, or, equivalently, the planetary index $k_{p}$. 


\subsubsection{Systematic and Random Errors in Sensor Data}

The other main contributors to error densities are the uncertainties in the sensor data. We discuss several classes of systematic and random errors in this report. Systematic errors should be treated explicitly. Our second set of recommendations is directed at reducing the systematic errors and inconsistencies in sensor weights and biases. We recommend the incorporation of methods for processing data from distant satellites that properly handle finite beam and accompanying track effects. We recommend inclusion of algorithms that bring the sensor weights into alignment with the actual observed values for any given satellite. Although not discussed here we have compared station parameters supplied to us with values determined by the Draper Laboratory. Several significant discrepancies exist between the two sets of station parameters, and we recommend the reconciliation of these discrepancies. These actions will benefit users of the SP model by reducing the overall errors and orbit wobble for eccentric orbits and by improving the stability of the orbit predictions.

\subsubsection{Stability, Model Selection, and Parameterization}

The third set of recommendations addresses issues related to failures such as spiking/shot noise and other difficulties encountered using the SP model. We have observed catastrophic and near-catastrophic failures that we believe are related to data quality and quantity and numerical stability. We cannot assume uniformly high data rates and quality for all orbits, nor can we assume that a single set of parameters is optimal for all orbit types. We recommend a further examination of the SP model and its internal parameters with the goal of finding settings that eliminate shot noise. These settings should be tested against groups of satellites with similar orbital and data characteristics to provide an estimate of the expected error distributions under these variations. In the difficult NCO and HEO regimes, semianalytic models such as DSST [19] and SALT [20] may be more stable against data degradation and for that reason may be preferable. We recommend the exploration of this possibility as well.

\subsubsection{Enabling Technologies}

The final set of recommendations involves taking advantage of enabling technologies to assist orbit analysts and operators of dedicated orbit propagator systems. We recommend the creation of a problem-solving environment (PSE) that links a front-end graphical user interface to back-end libraries of astrodynamics routines. This PSE would incorporate artificial intelligence technologies such as expert systems to automate the decision process of selecting appropriate orbital models and parameters, given the heterogeneous collections of orbit regimes and data rates encountered in practice. Such a system could automatically optimize the code parameters for the orbit of interest, handle quantities such as F10.7 solar flux and geomagnetic index, automatically select the best model and integration scheme for the data acquisition rates, and automatically provide an assessment of the quality of the resulting estimation. 


\section{ACKNOWLEDGEMENTS}

We thank Paul Cefola and Ron Proulx of Charles Stark Draper Laboratory their helpful comments and assistance with workstation GTDS, Bill Barker and Steve Casali of ITT Industries for their assistance with sensor and sequencing issues, and Kitt Carlton-Wippern for his unique dedication to and enthusiastic support for this project. This work was sponsored by the Air Force Materiel Command's Electronic Systems Center, Detachment 5 (AFMC/ESC Det 5) SMC under U.S. DOE/USAF Interagency Agreement 1910-G055-A1. Particular thanks are due AFMC/ESC's Mr. David J. Steele, Director of the Strategic \& Nuclear Deterrence Command and Control System Program Office for his executive oversight; Mr. David B. Rowe, Space Requirements Lead (the designated Quality Assurance Evaluator), for his technical direction and advice; and Mr. Keith L. Nutt, the Program Manager, for his administrative oversight throughout the study. 


\section{References}

1. N. L. Johnson, "Monitoring and Controlling Debris in Space," Scientific American 279:2, 62-67 (1998).

2. K. T. Alfriend et al., "Probability of Collision Error Analysis," AIAA/AAS Astrodynamics Specialist Conference, Houston, Tex., Aug. 10-12, 1998.

3. Goddard Space Flight Center, Mathematical Theory of the Goddard Trajectory Determination System, X-58276-77, ed. J. O. Cappellari et al 1976.

4. Goddard Space Flight Center, Goddard Trajectory Determination System (GTDS) Mathematical Theory (Rev. 1), FDD/552-89/0001 and CSC/TR-89/6001, ed. A. C. Long et al., 1989.

5. Cefola, P. (1992), R\&D GTDS semianalytical satellite theory input processor. Draper Laboratory IOC ESD-92582/SGI GTDS-92-001.

6. Metzinger, R. W. (1993), Validation of the workstation version of R\&D GTDS. Draper Laboratory memorandum, available from P. Cefola, CSDL.

7. T. D. Platt and L. E. Herder, Ranking Satellite Propagators: A Statistical Approach, proceedings of the $13^{\text {th }}$ MIT/LL Space Surveillance Workshop, Lexington, Mass., March 28-30, 1995.

8. R. N. Wallner et al., The Accuracy of General Perturbations and Semianalytic Satellite Ephemeris Theories, proceedings of the 13th MIT/LL Space Surveillance Workshop, Lexington, Mass., March 28-30, 1995.

9. Kaman Sciences Corporation, Space Station Debris Avoidance Study Final Report, KSPACE 97-47, Kaman Sciences Corporation, Colorado Springs, Colo., 1997.

10. K. C. Carlton-Wippern, Satellite Position Dilution of Precision (SPDOP), Paper AAS 97-609, AAS/AIAA Astrodynamics Specialist Conference, Sun Valley, Idaho, Aug. 4-7, 1997.

11. D. A. Vallado, Fundamentals of Astrodynamics and Applications, New York, McGraw-Hill, 1997.

12. K. C. Carlton-Wippern, Probability of Collision in Space, Paper AAS 97-639, AAS/AIAA Astrodynamics Specialist Conference, Sun Valley, Idaho, Aug. 4-7, 1997.

13. M. Beckerman and E. M. Oblow, "Treatment of Systematic Errors in the Processing of Wide Angle Sonar Sensor Data for Robotic Navigation," IEEE Trans. Robot. Automat RA-6, 137-145 (1990).

14. J. de Lafontaine, Orbital Dynamics in a Stochastic Atmosphere and a Nonspherical Gravity Field, PhD Thesis, University of Toronto, 1986.

15. A. E. Hedin, "Extension of the MSIS Thermosphere Model into the Middle and Lower Atmosphere," $J$. Geophys. Res. 96: A2, 1159-1172 (1991).

16. C. C. Chao et al., "An Evaluation of Jacchia 71 and MSIS 90 Atmosphere Models with NASA ODERACS decay data," J. Astronaut. Sci. 45, 131-141 (1997).

17. F. Berlier et al., "A Thermosphere Model Based on Satellite Drag Data," Anneles de Geophysics 43, 9-24 (1978), and pp. 21-25 in Proceedings of the European Geophysical Society General Meeting, Vienna, Austria, 1997.

18. M. Beckerman, Adaptive Cooperative Systems, New York, Wiley, 1997.

19. R. J. Proulx et al., A Theory for the Short-Periodic Motion Due to the Tesseral Harmonic Gravity Field, AAS Paper 81-180, AAS/AIAA Astrodynamics Specialist Conference, Lake Tahoe, Nev., August 1981.

20. J. J. F. Liu and R. L. Alford, A Semianalytic Theory for the Motion of a Close-Earth Artificial Satellite with Drag, AIAA/AAS preprint 79-0123, presented at the 17th Aerospace Sciences Meeting, New Orleans, La., 1979. 
APPENDIX A:

PREDICTION ERRORS 

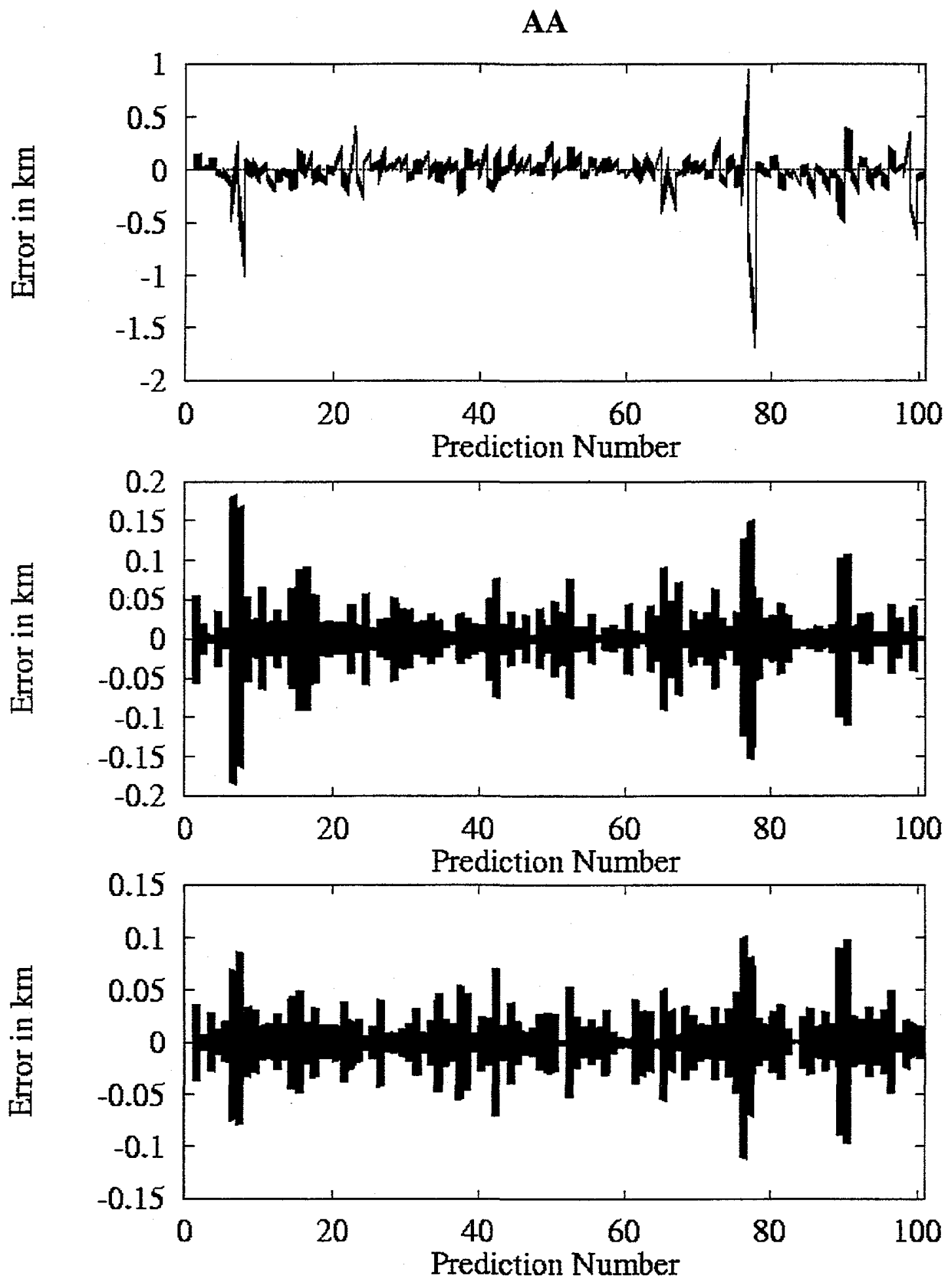
BB
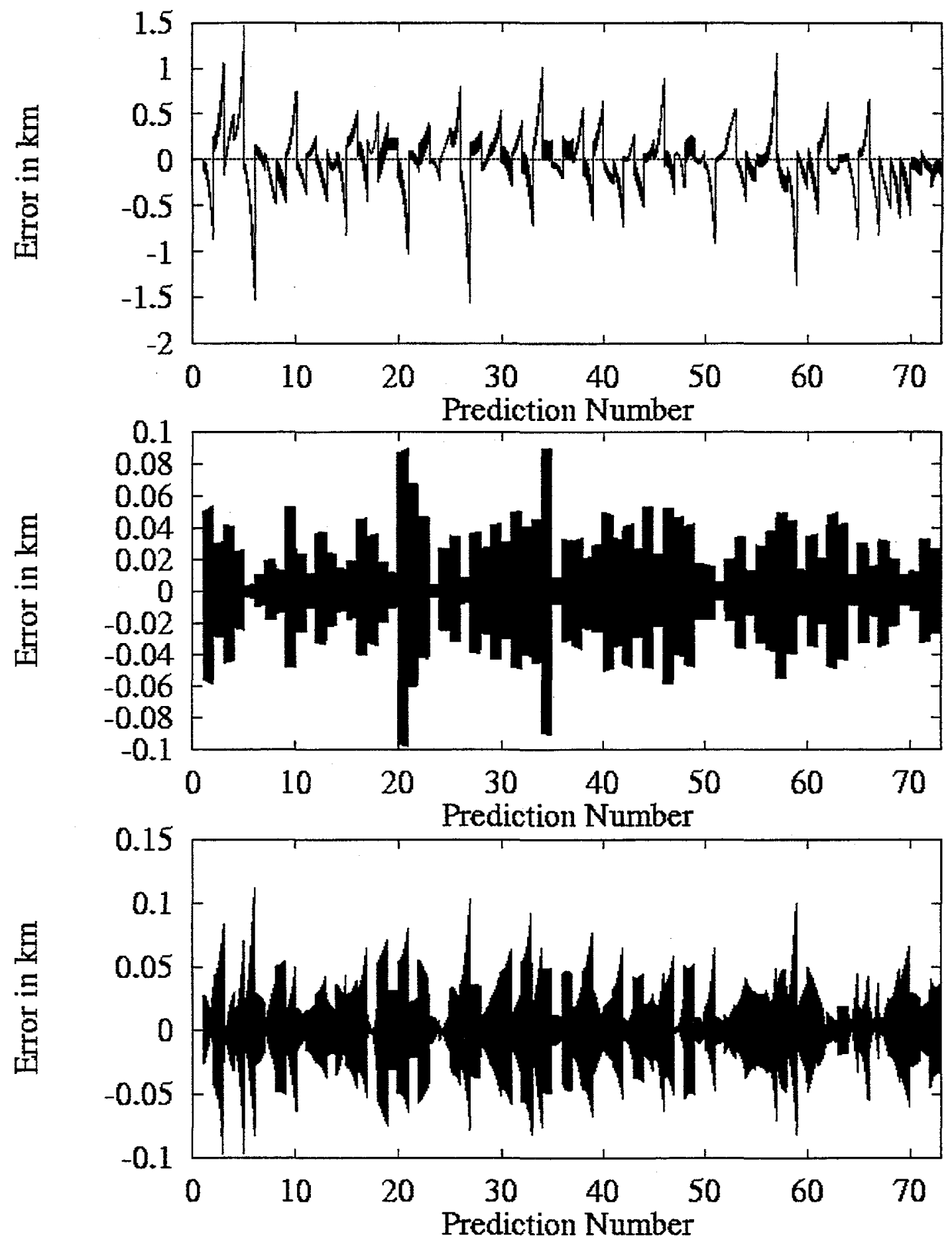
CC
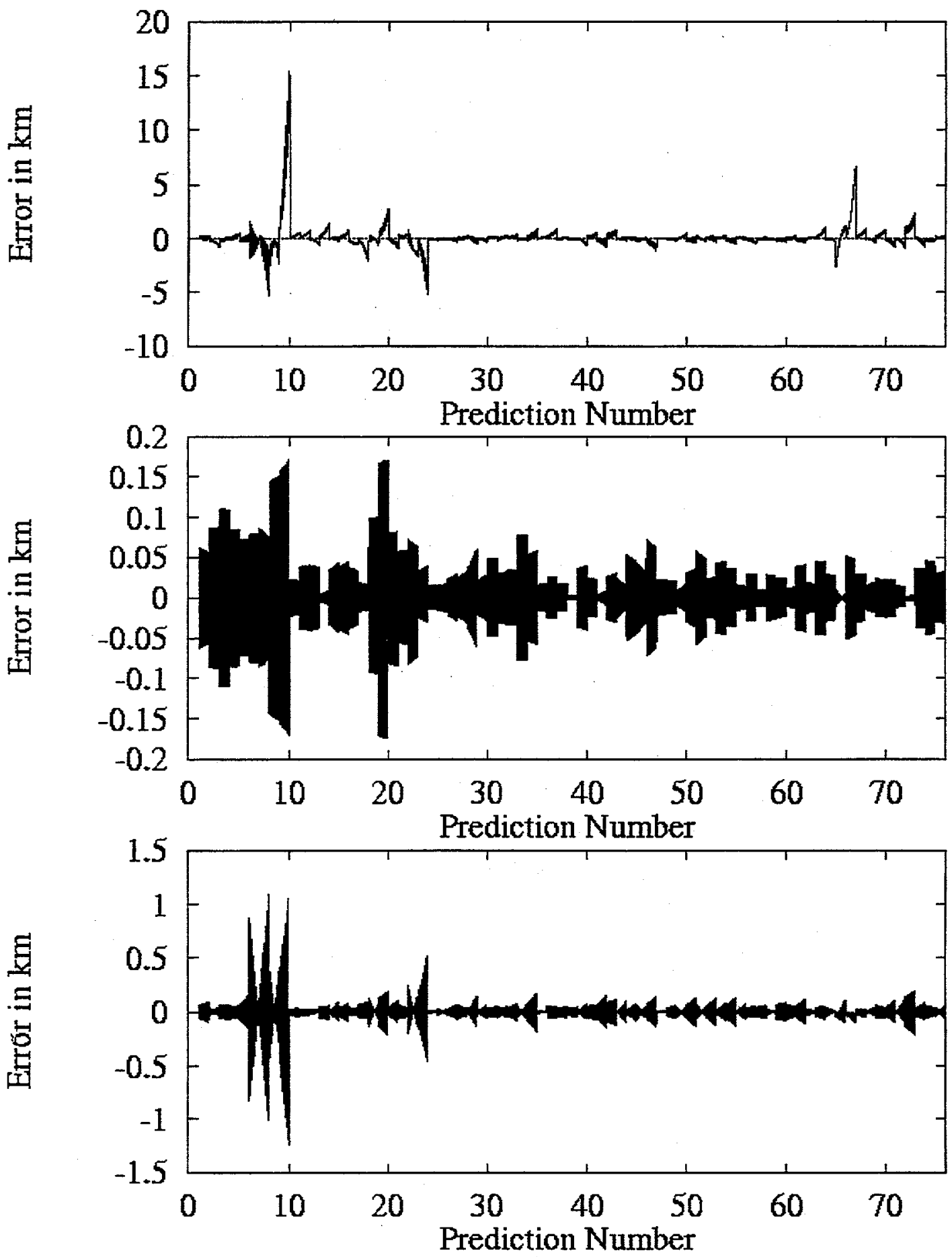

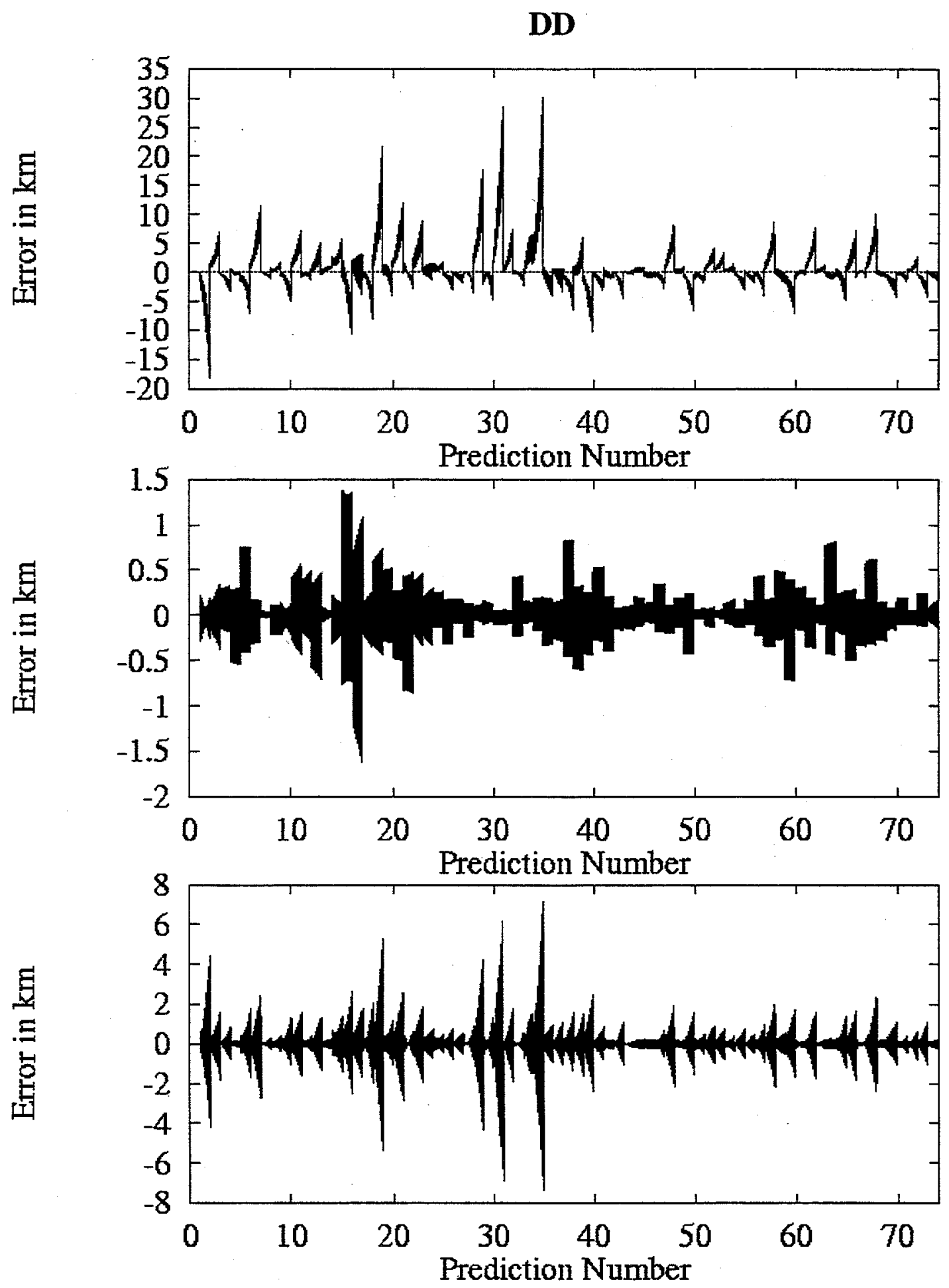
EE
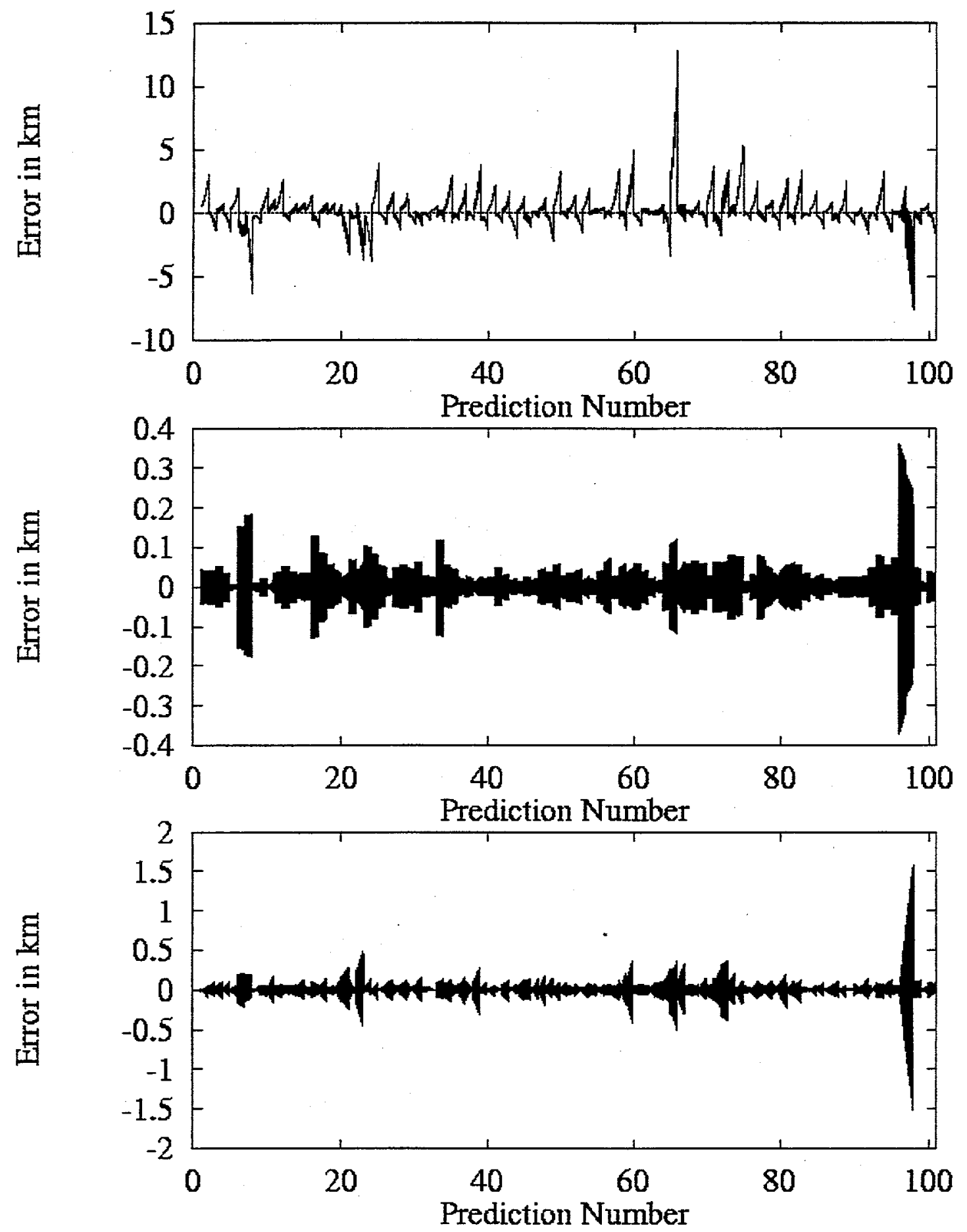

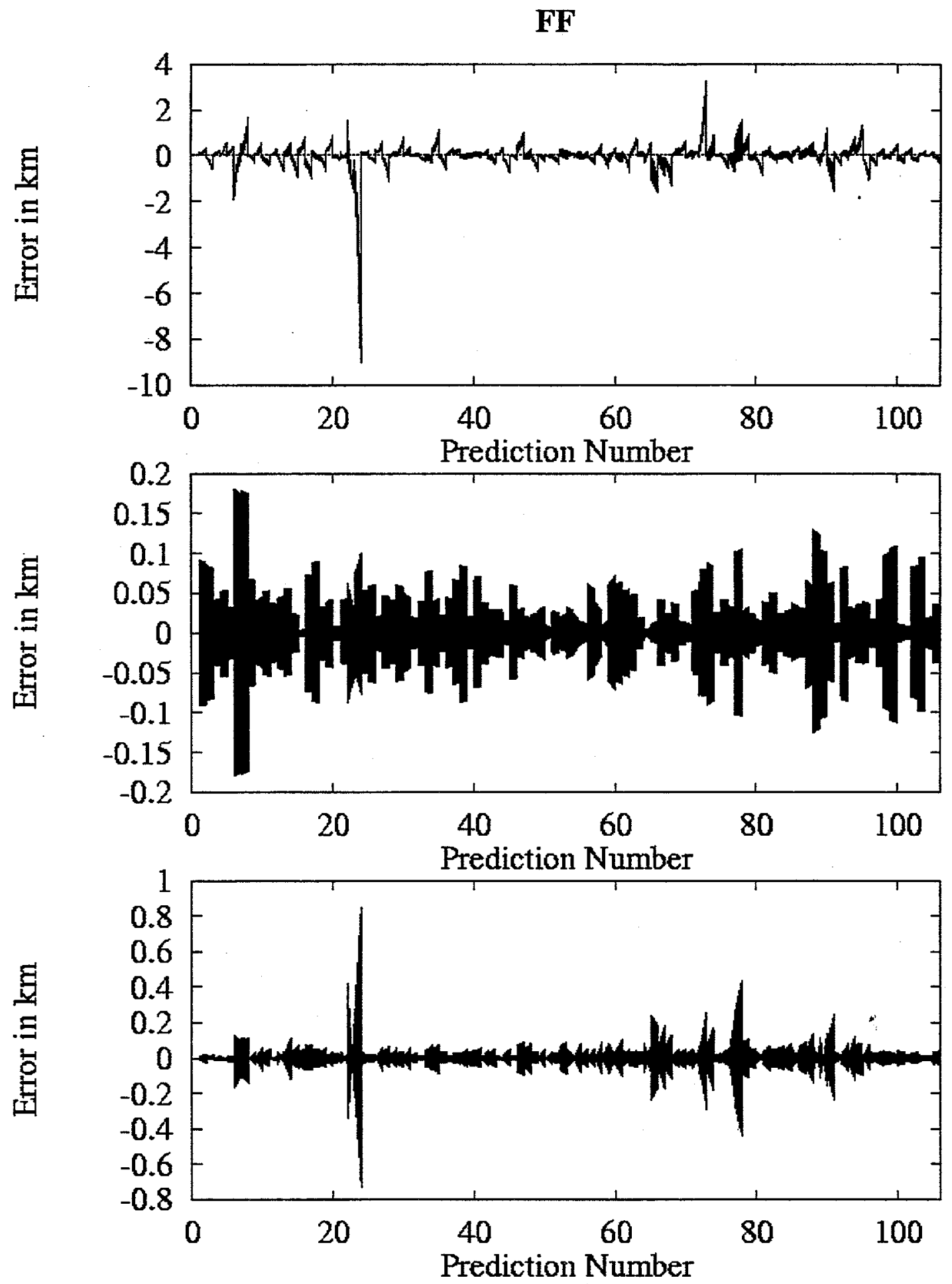
GG
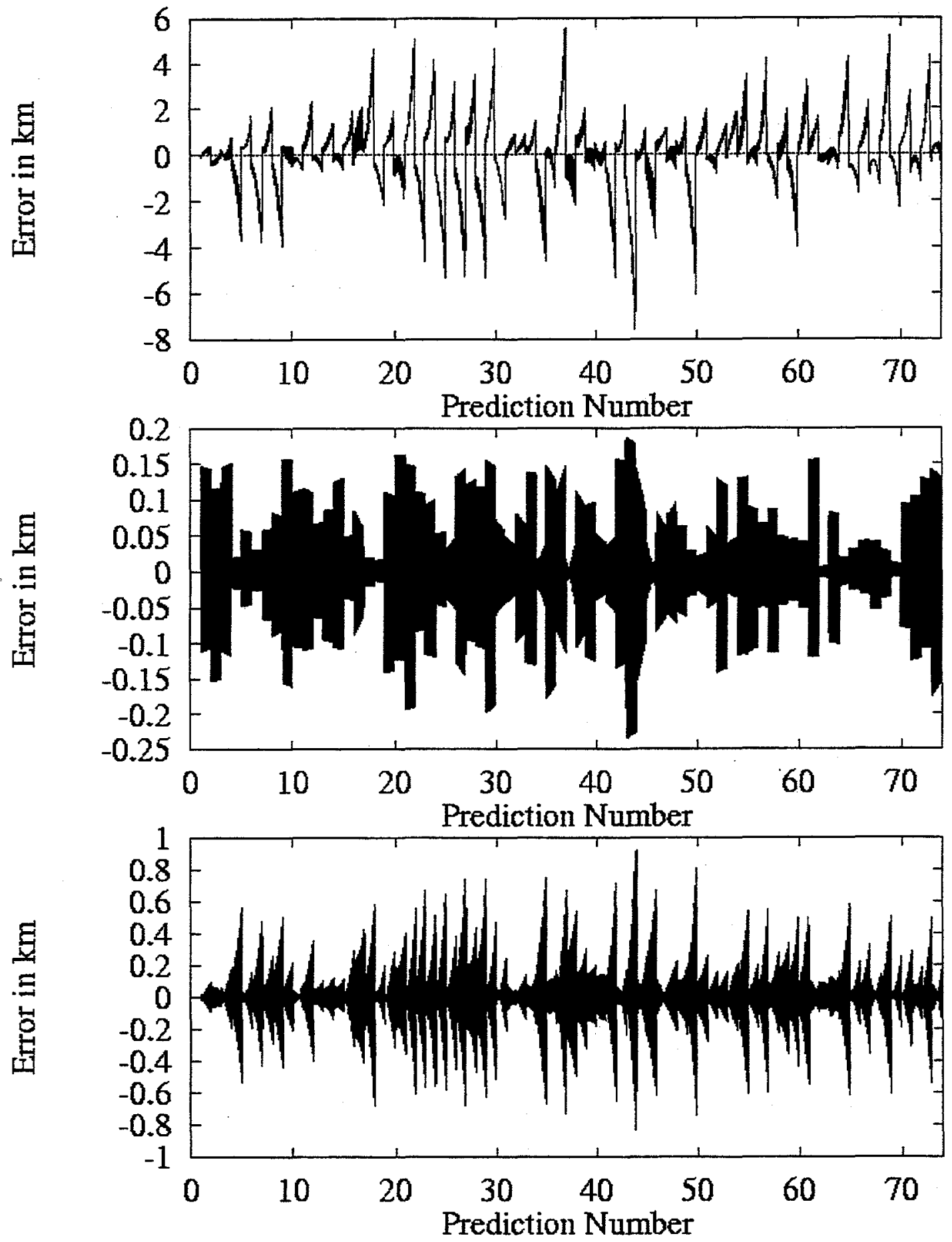


\section{HH}
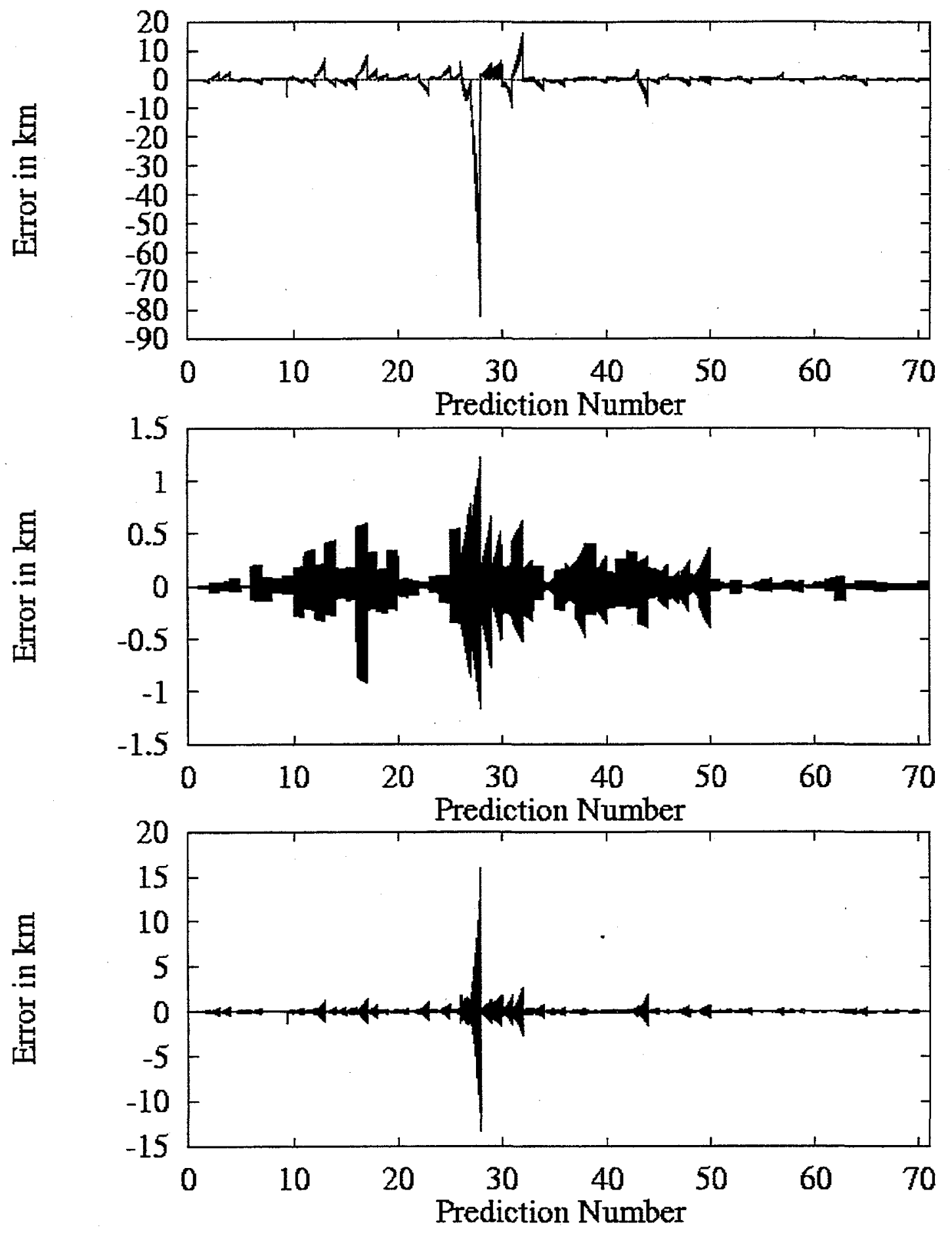
II
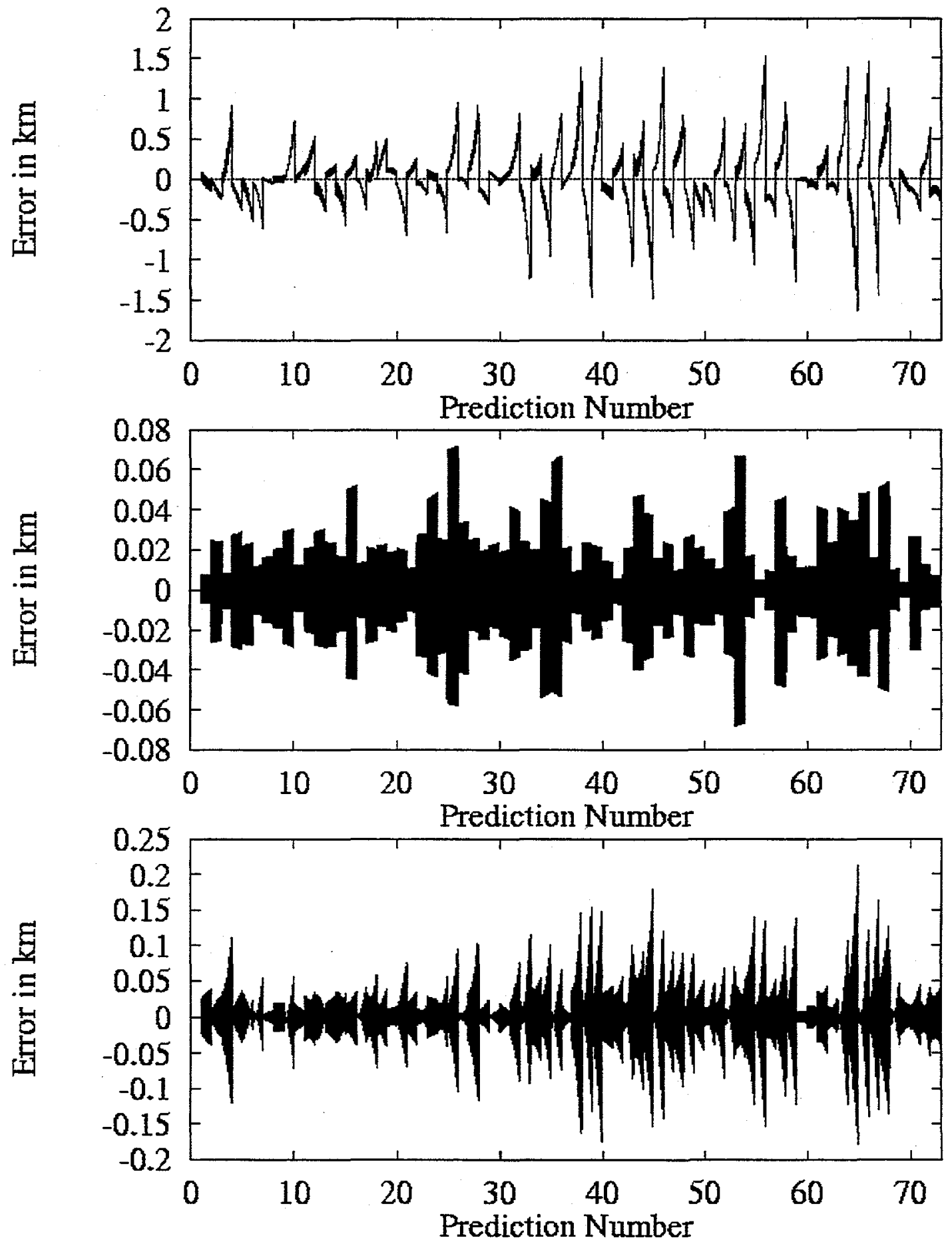

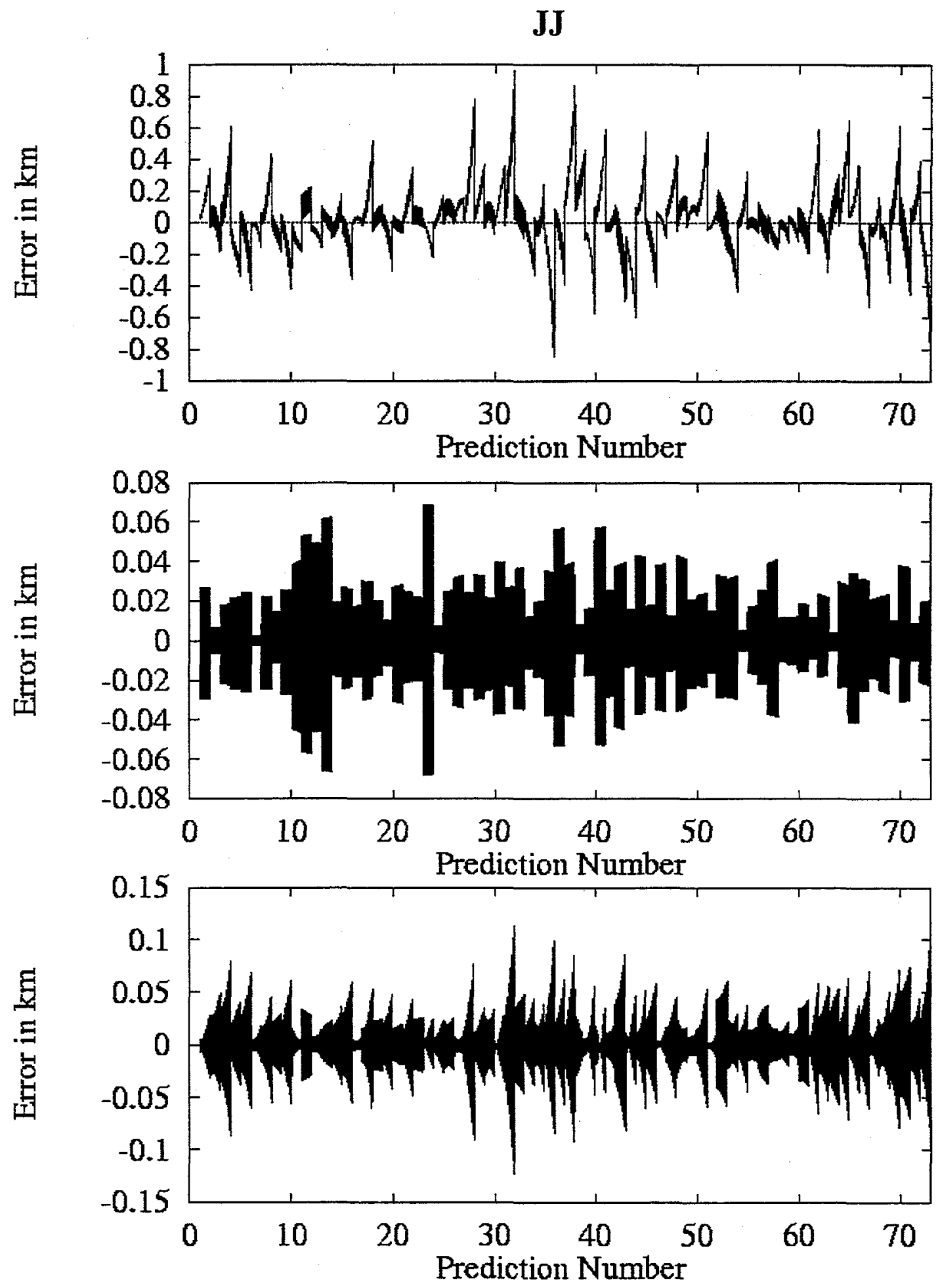
KK
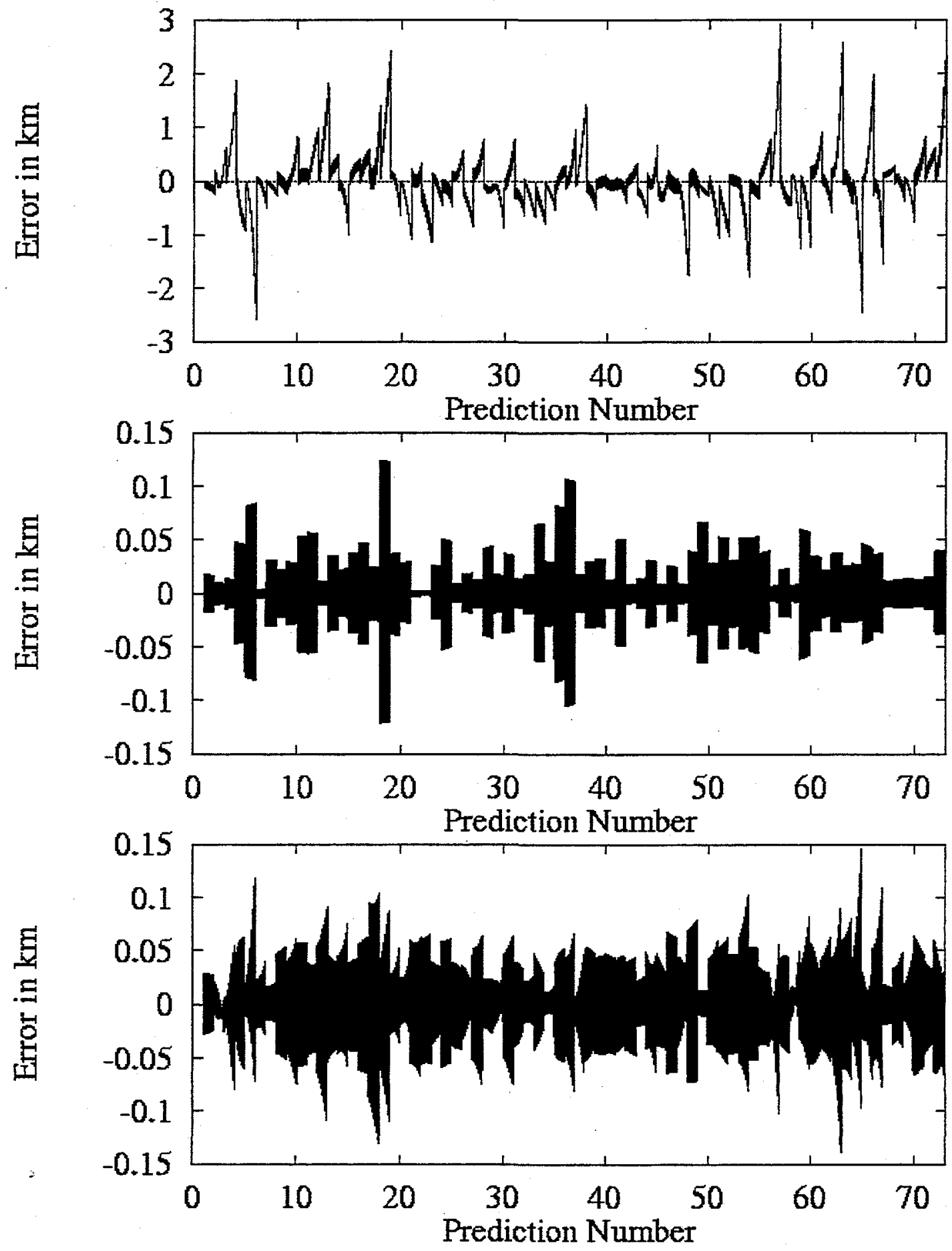
LL
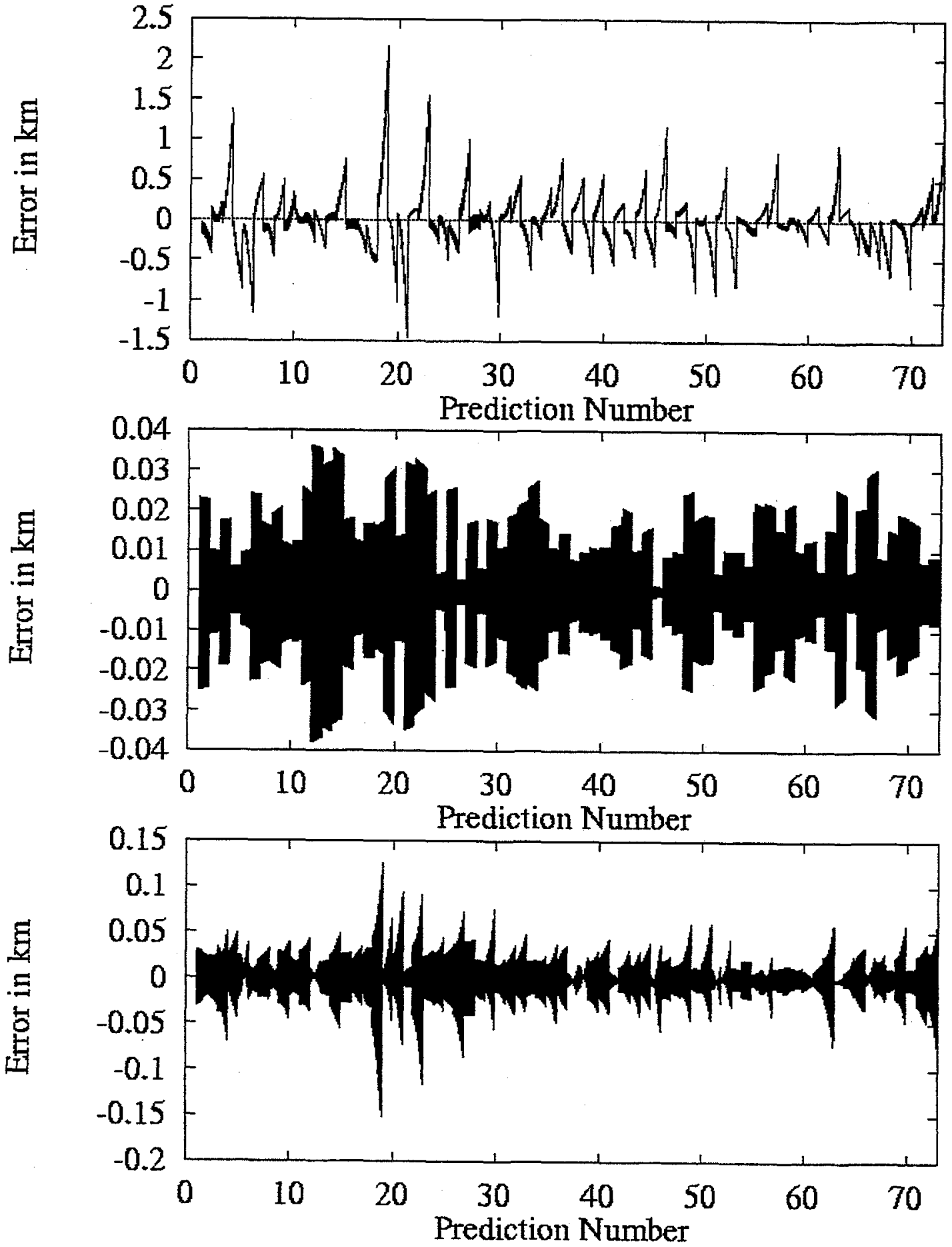
MM
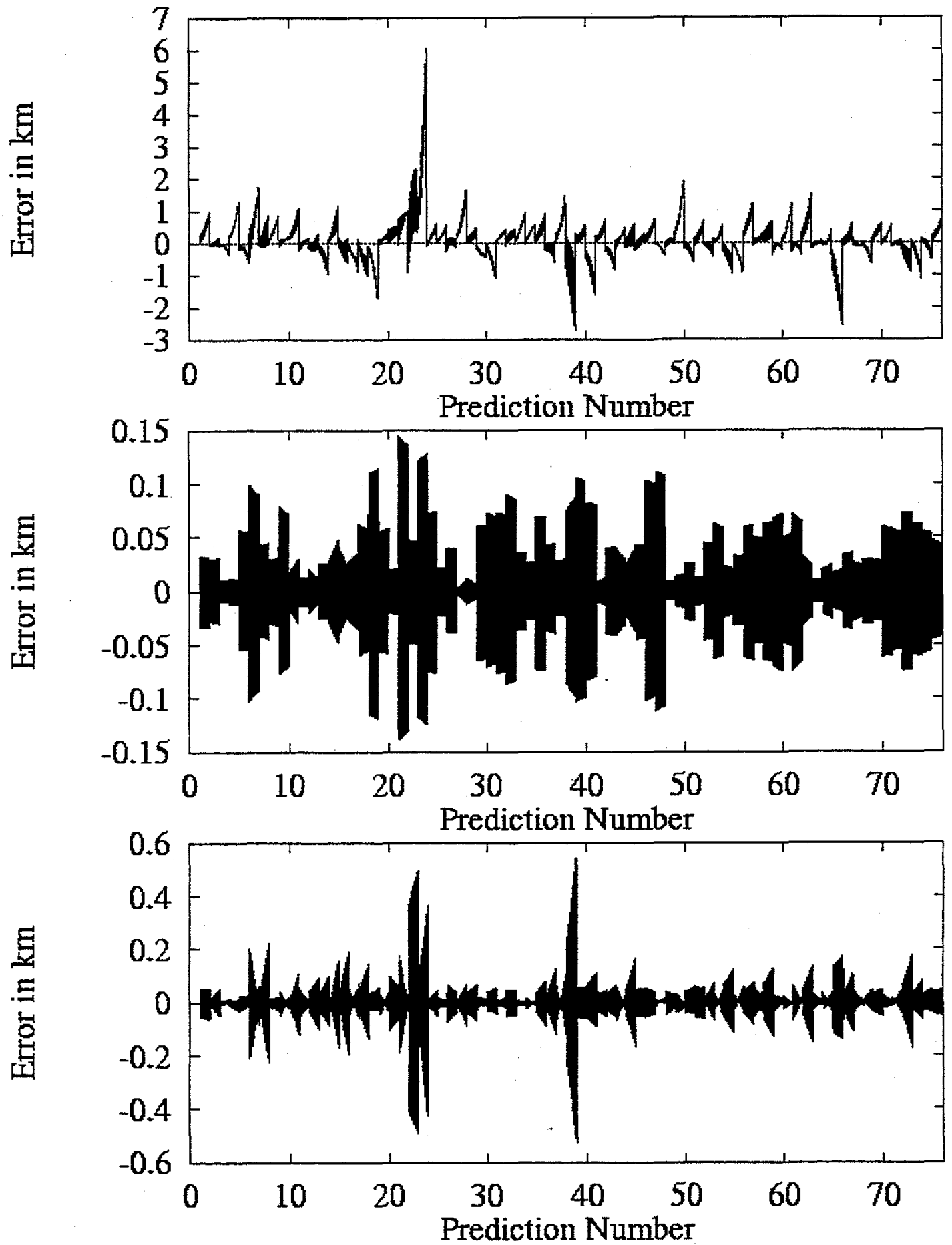

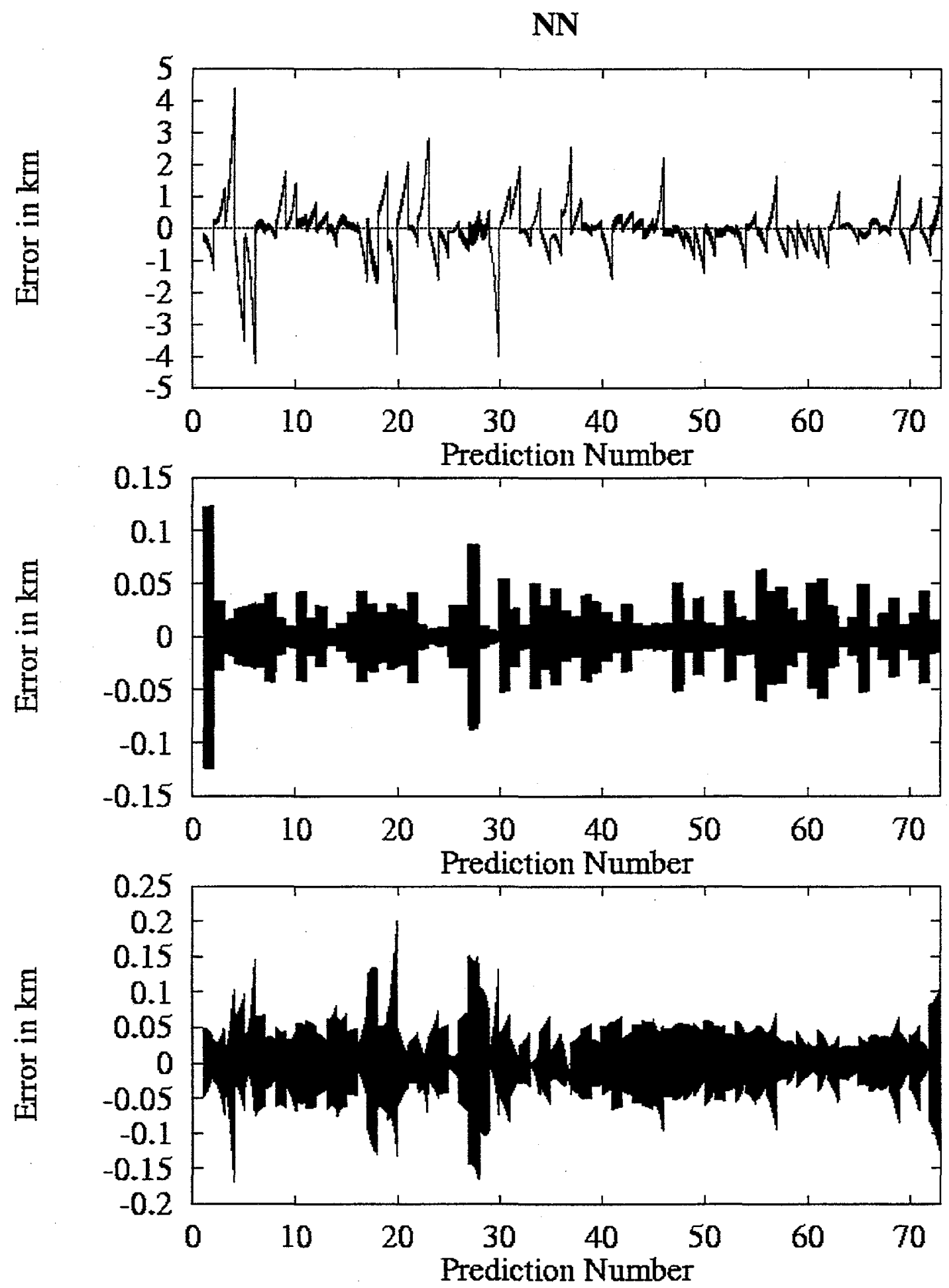

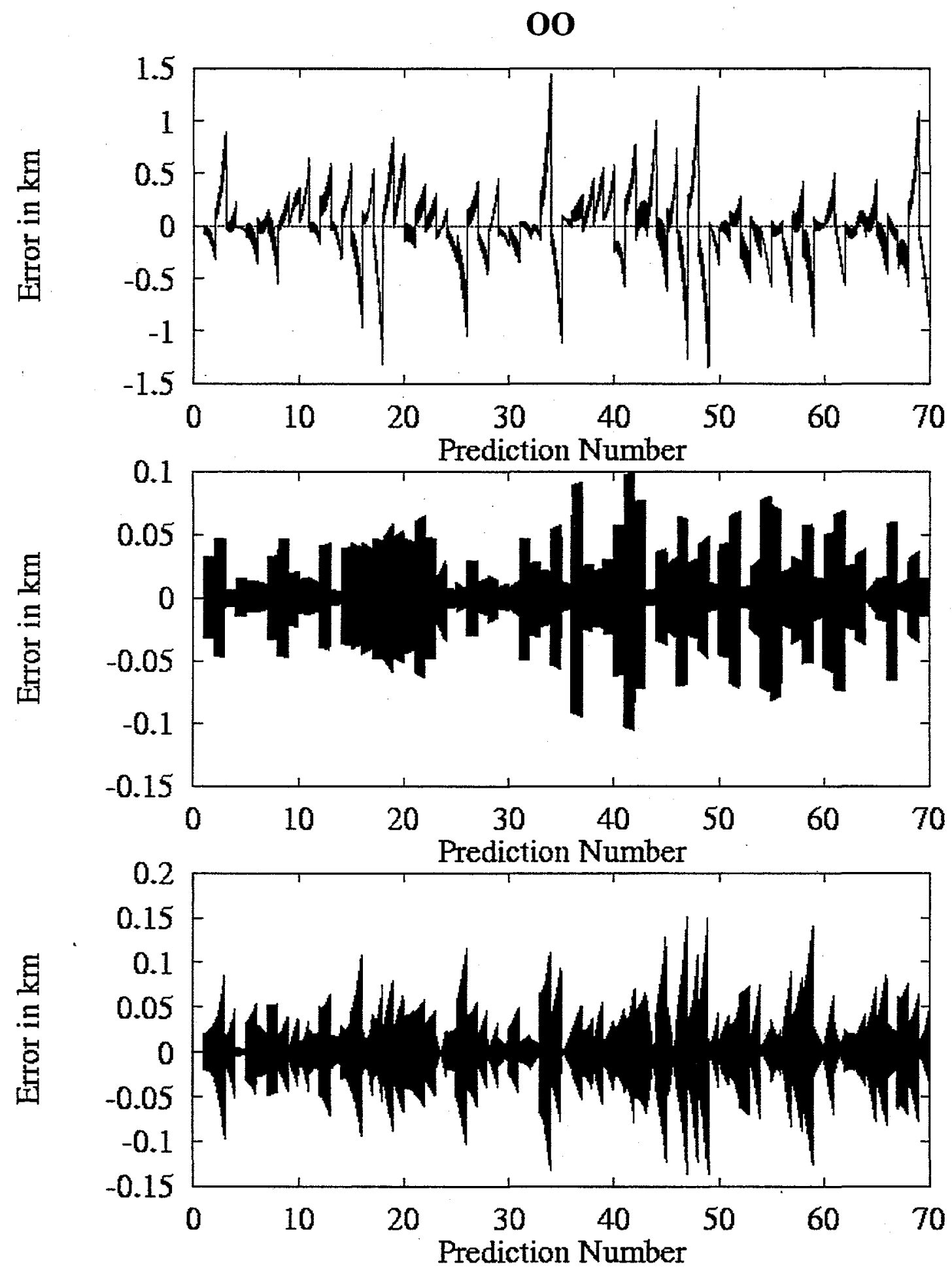

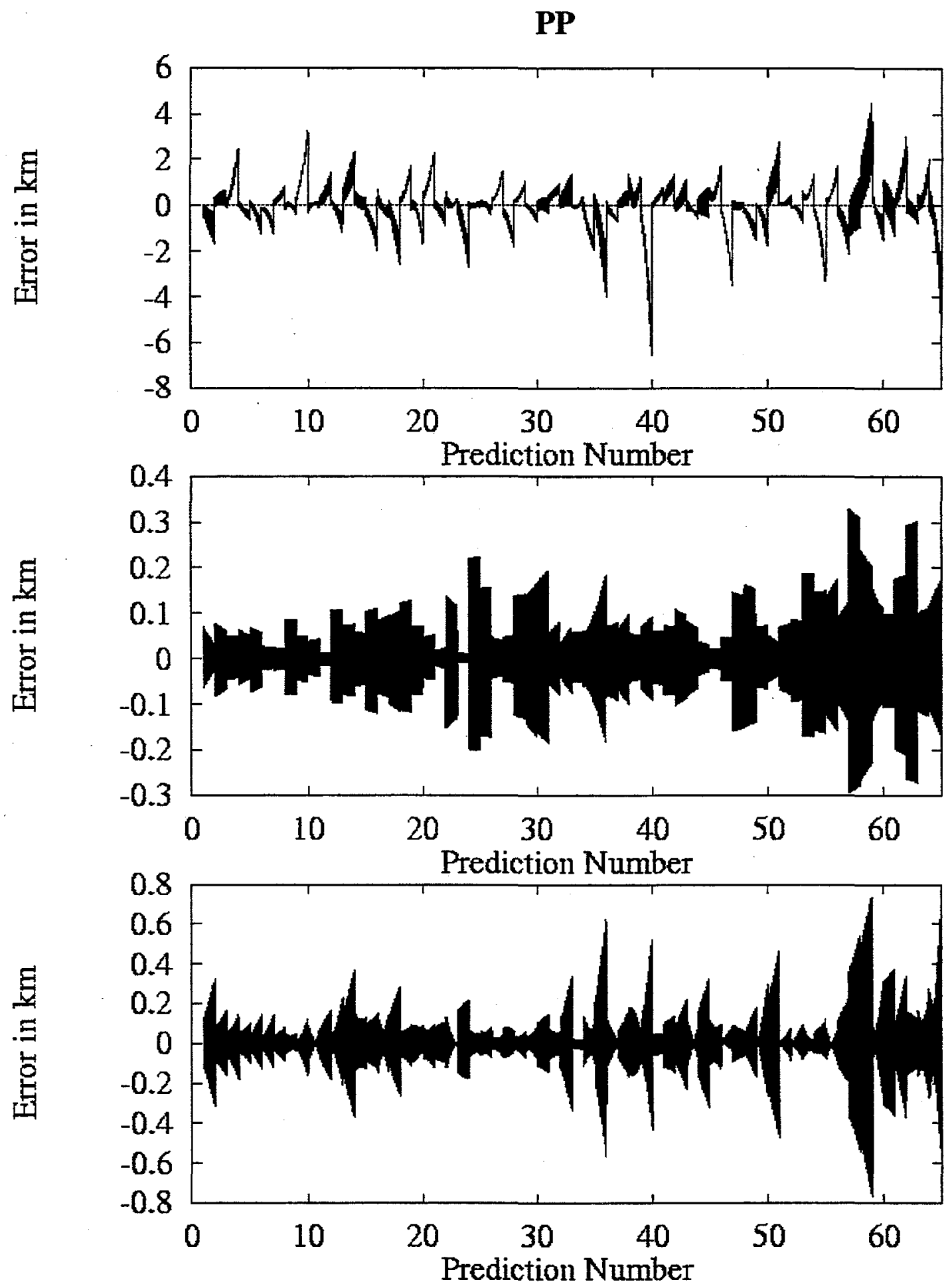

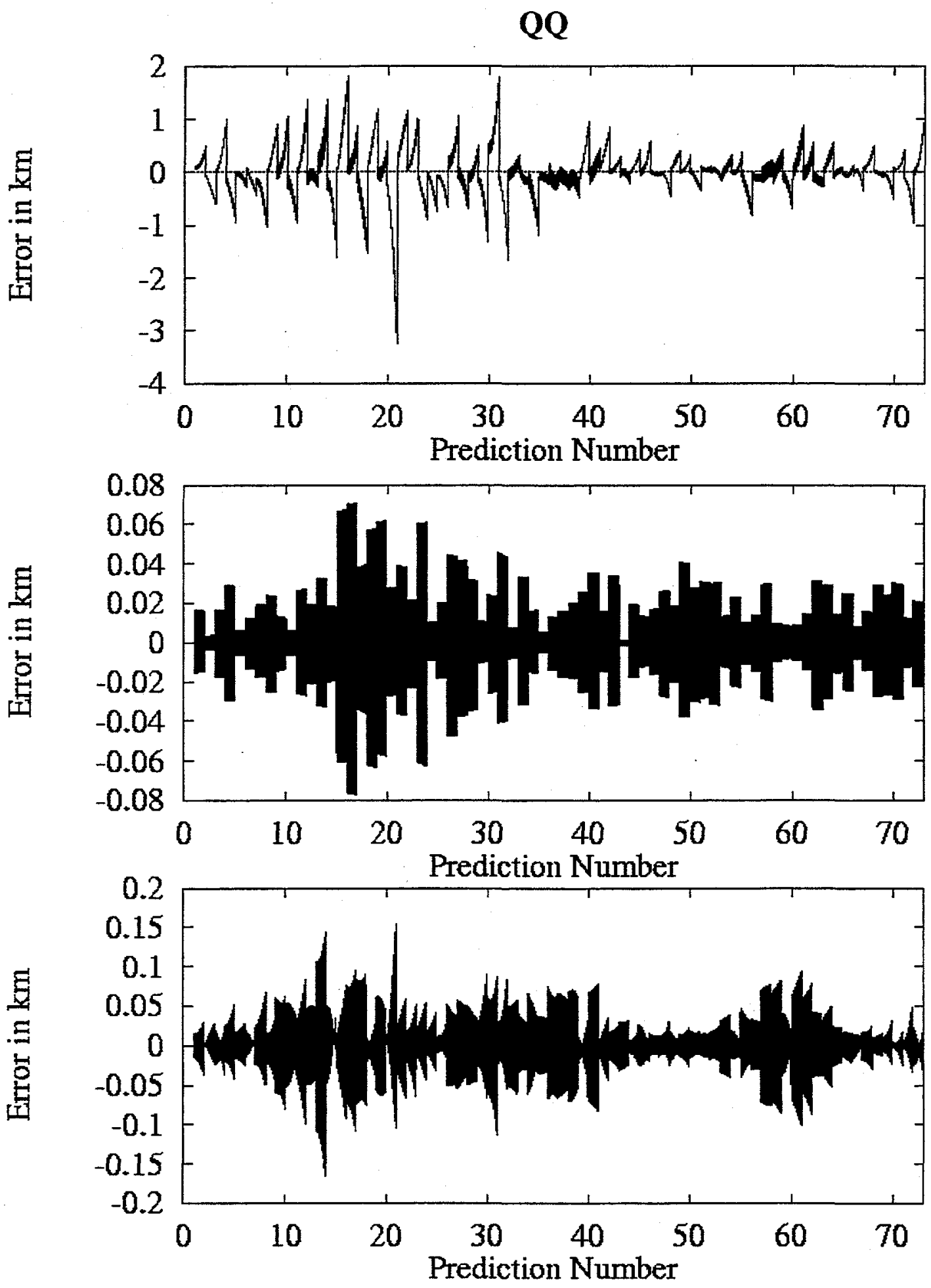

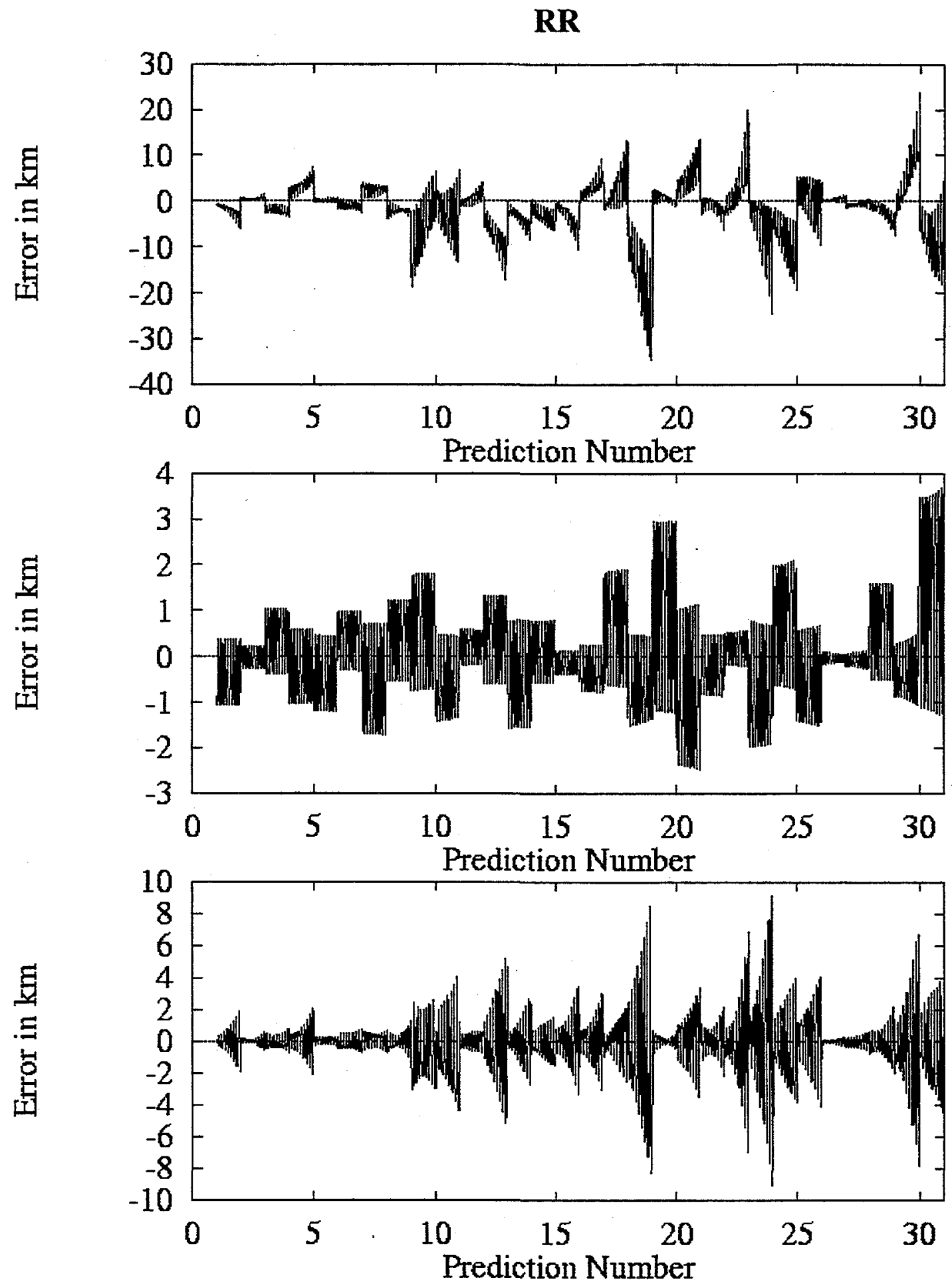

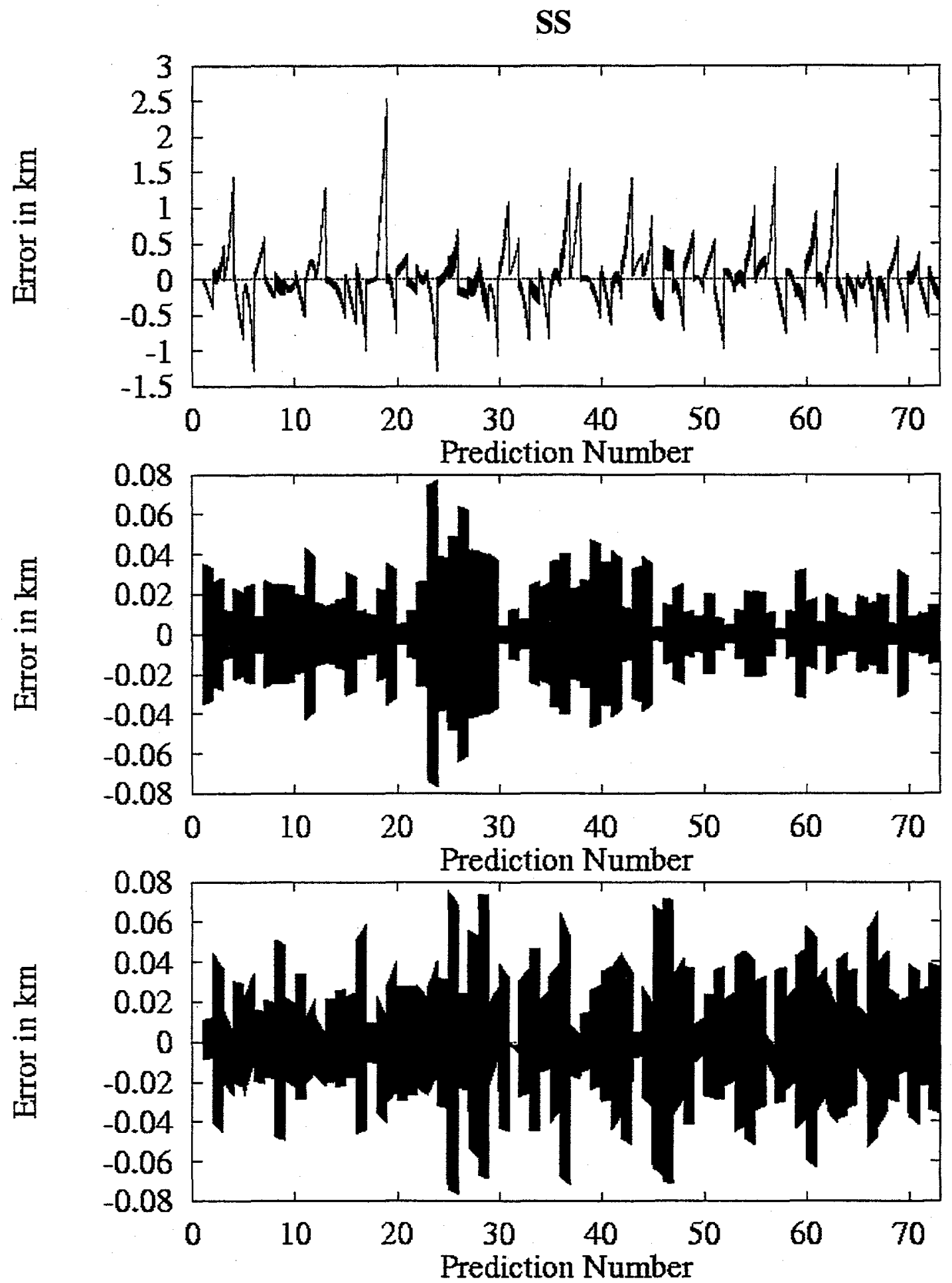
TT

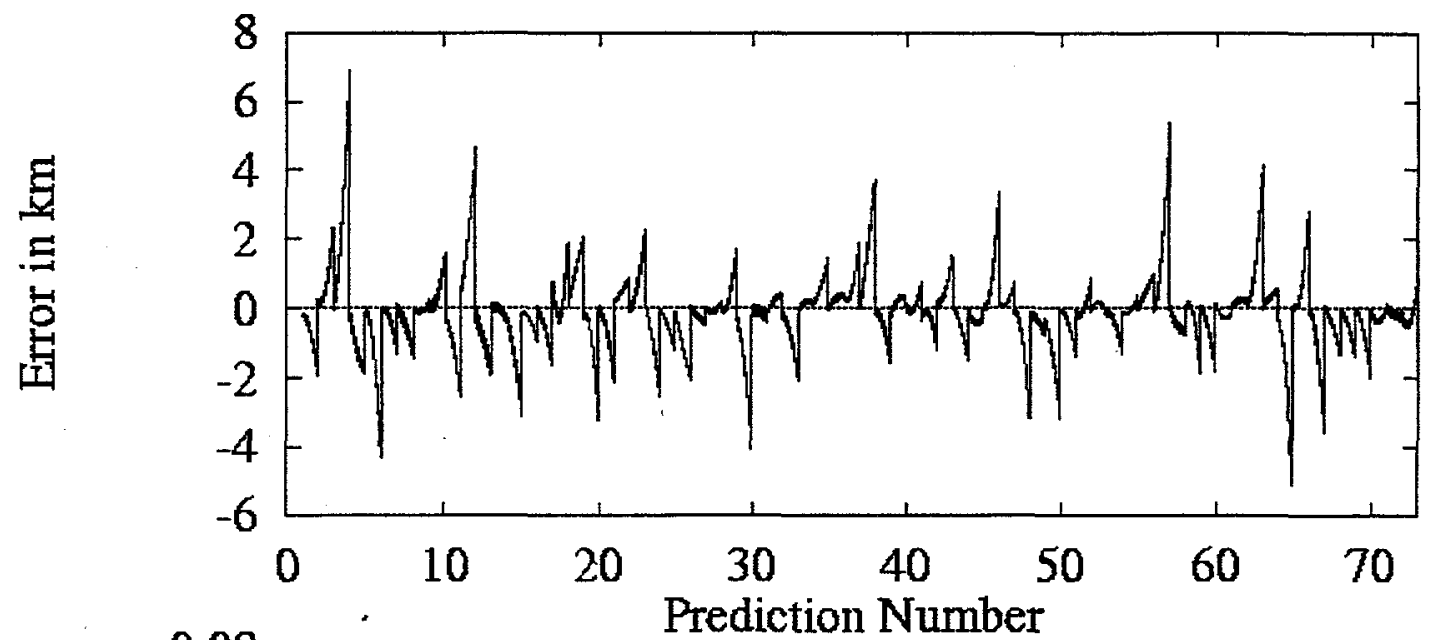

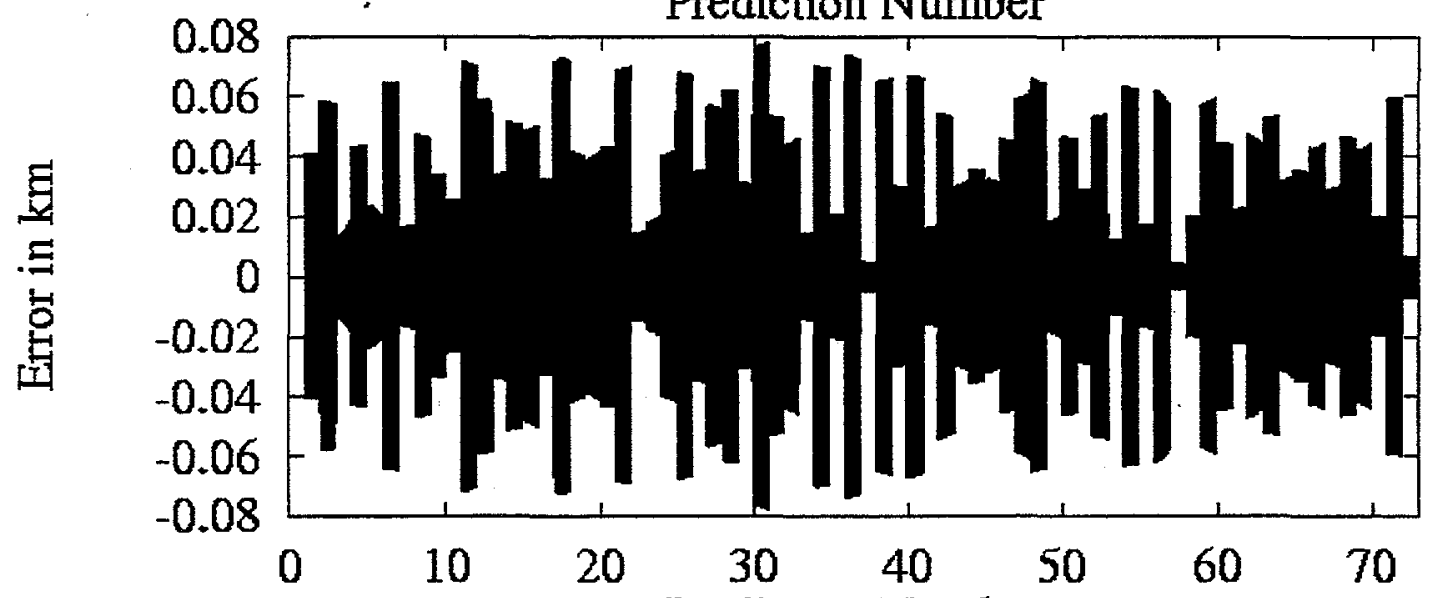

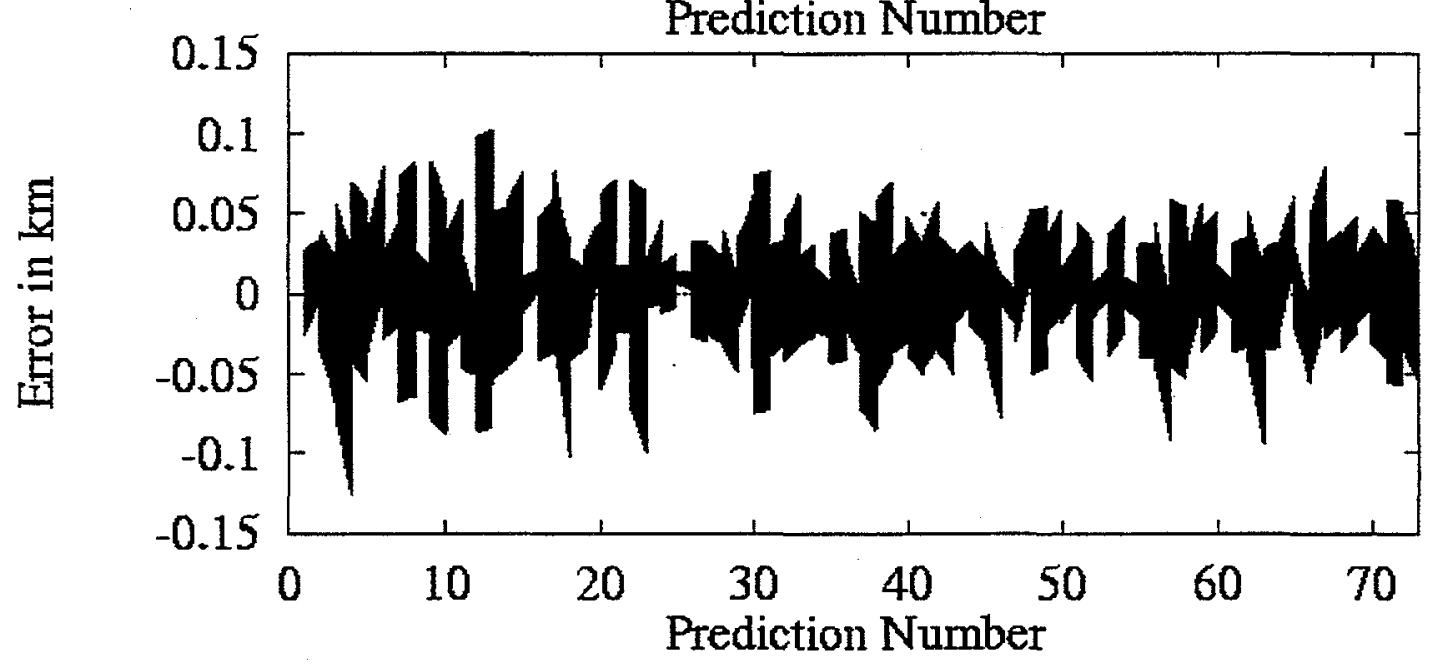


UU
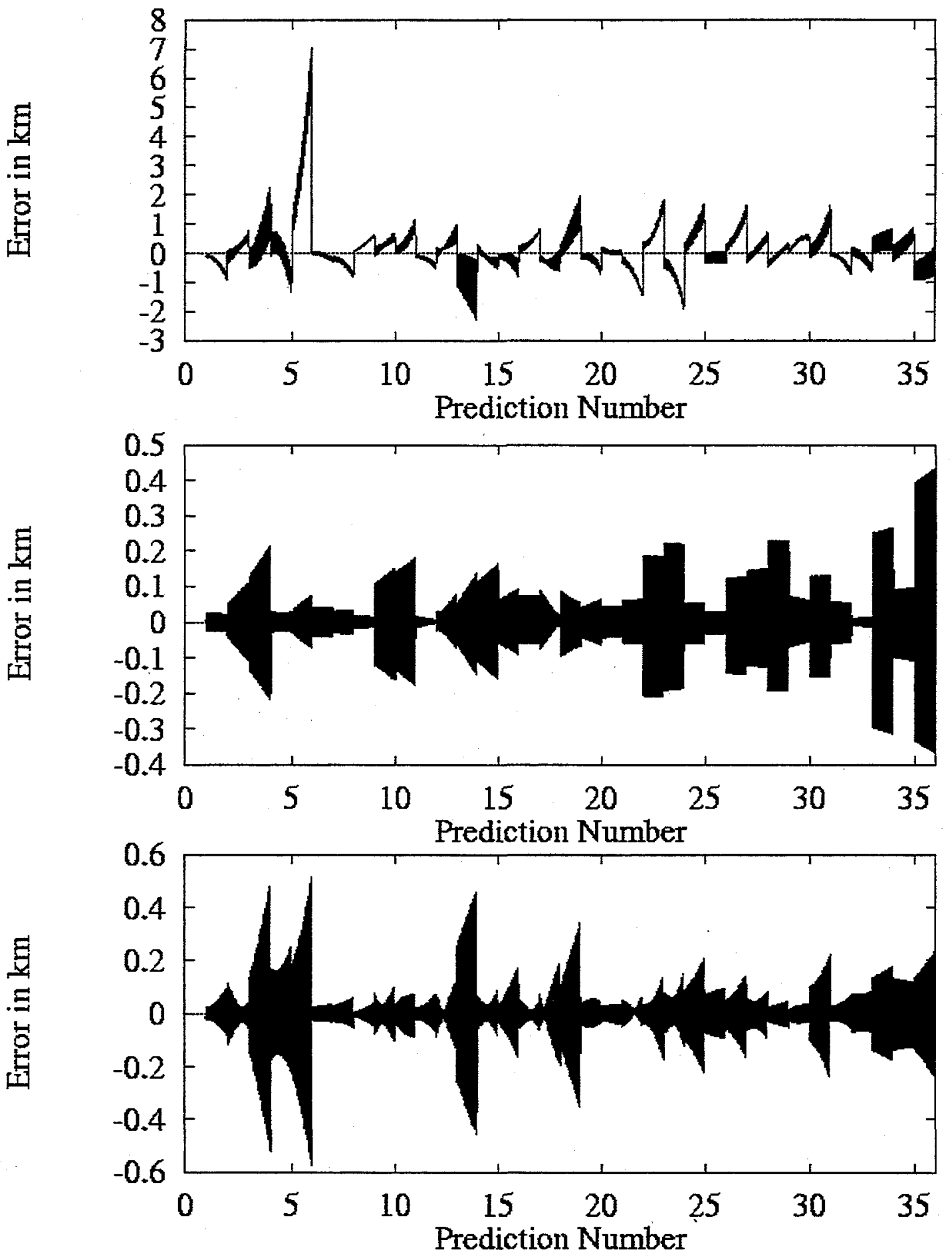

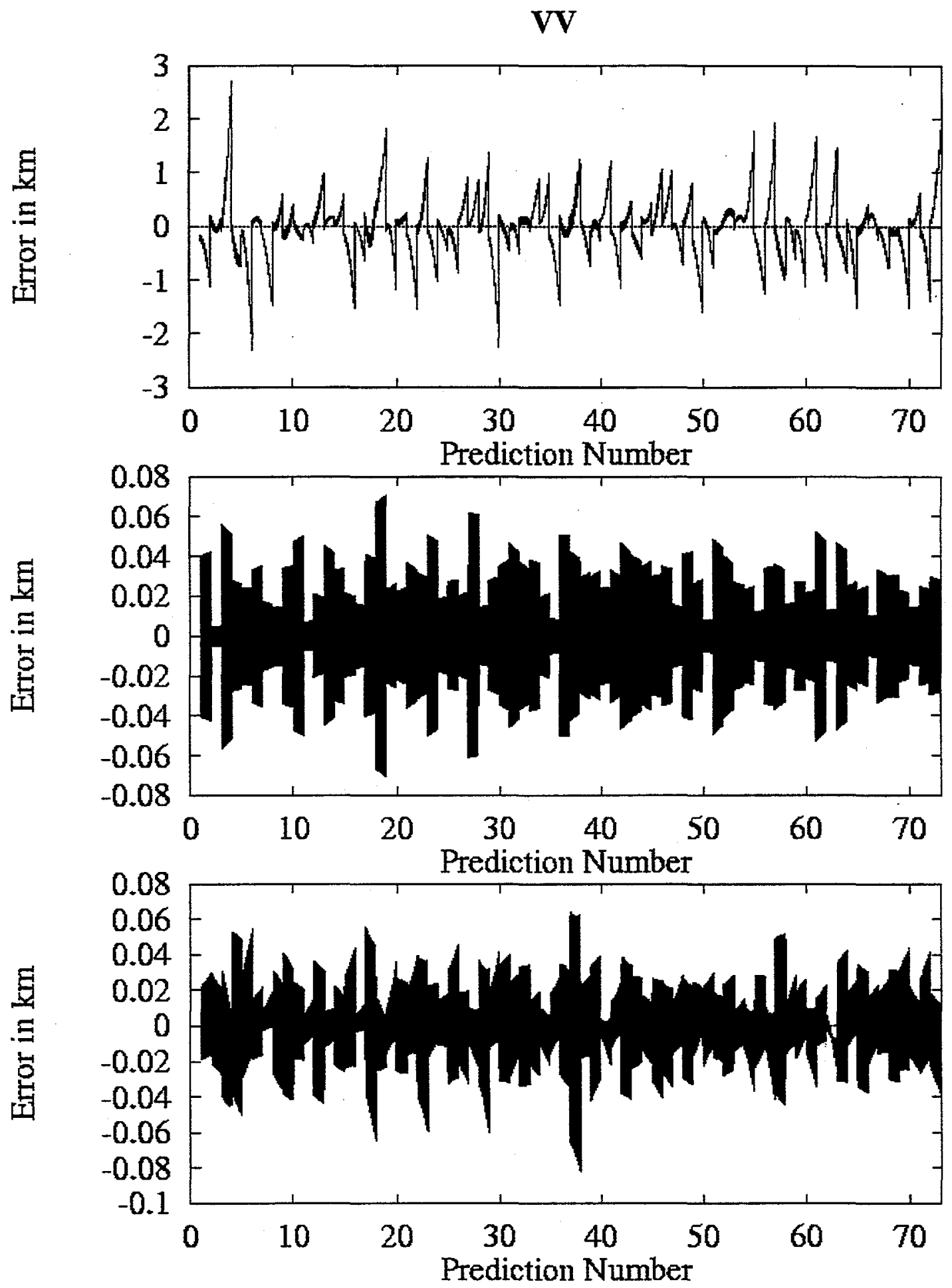

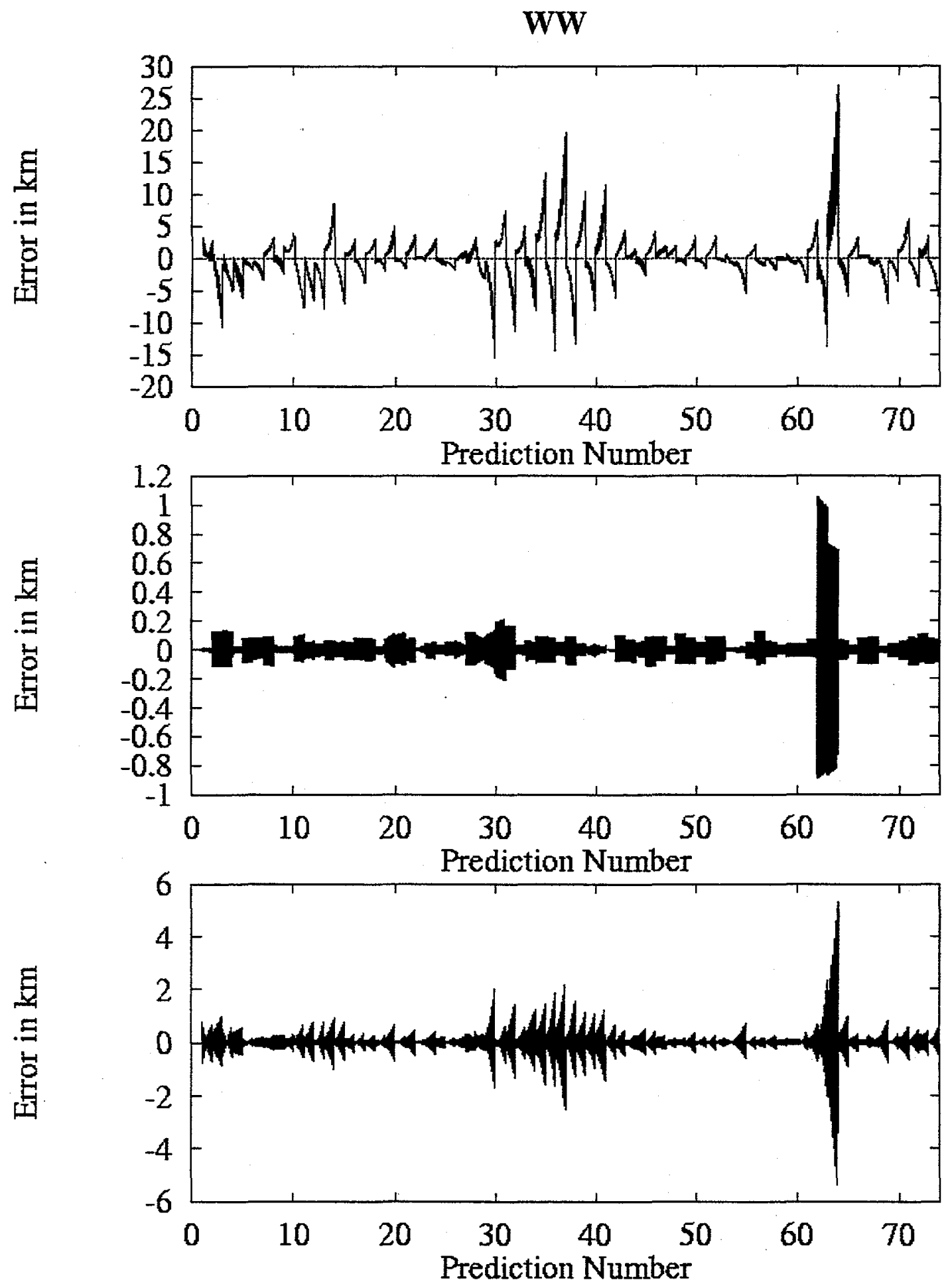

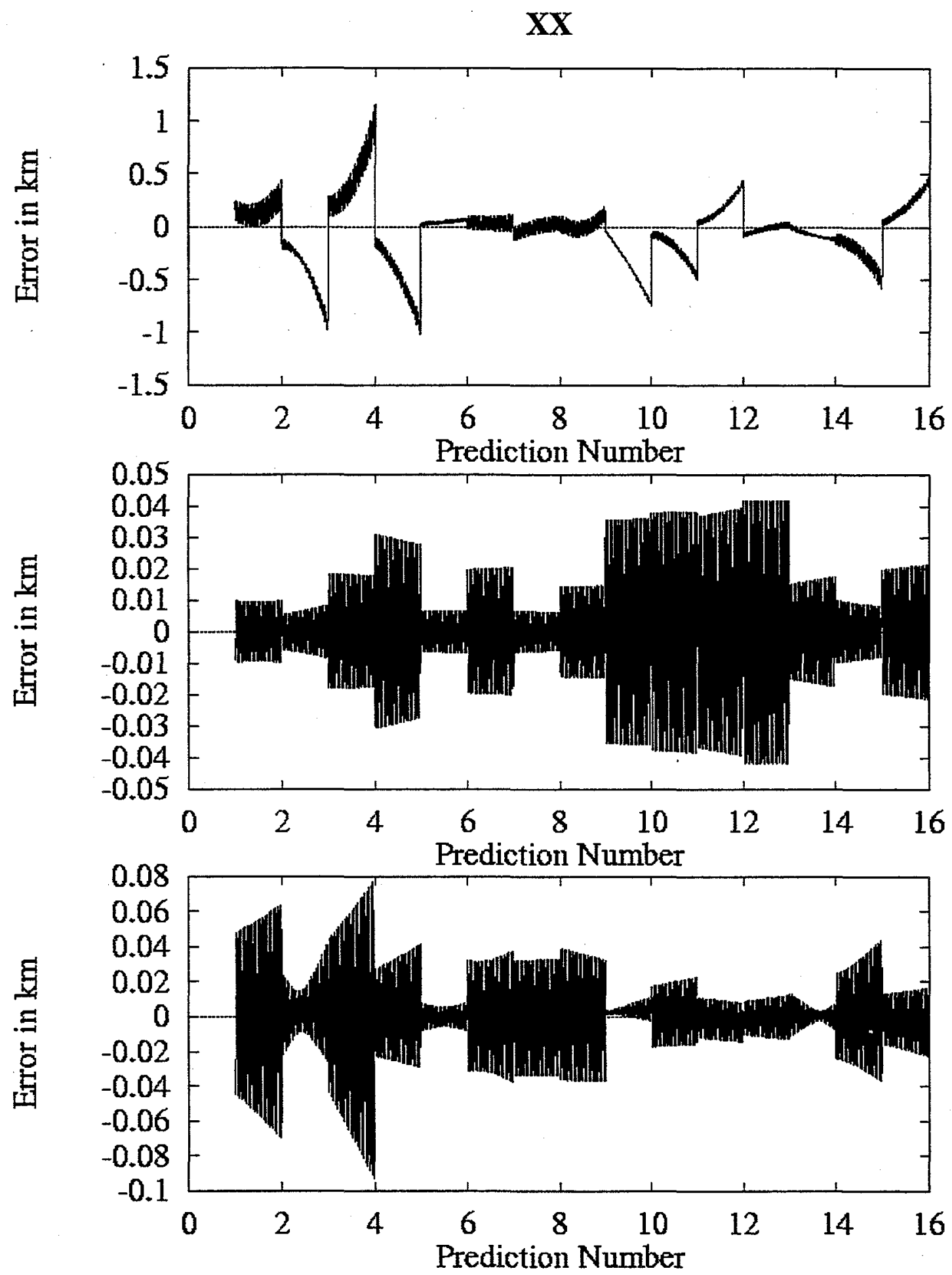
YY
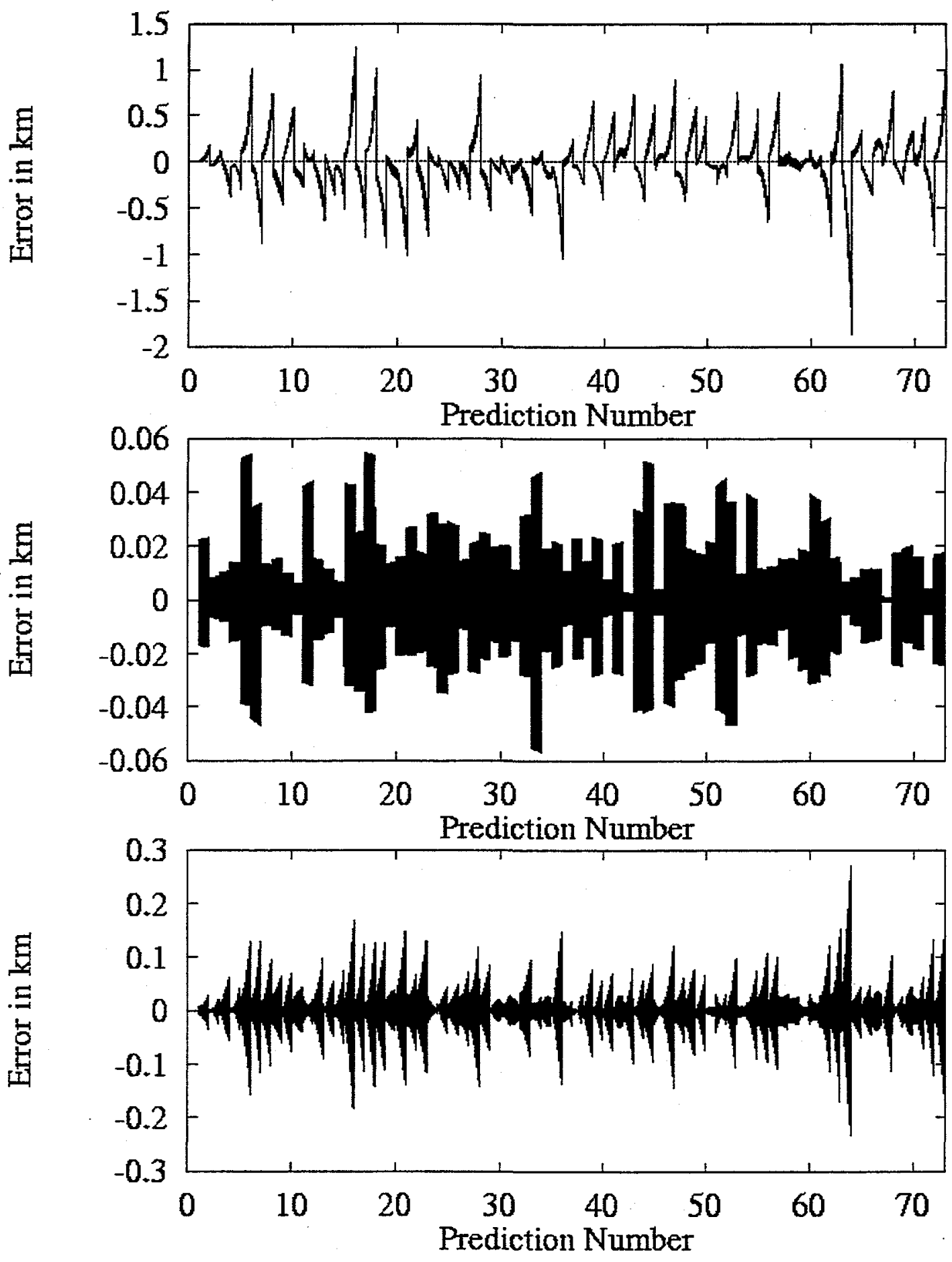

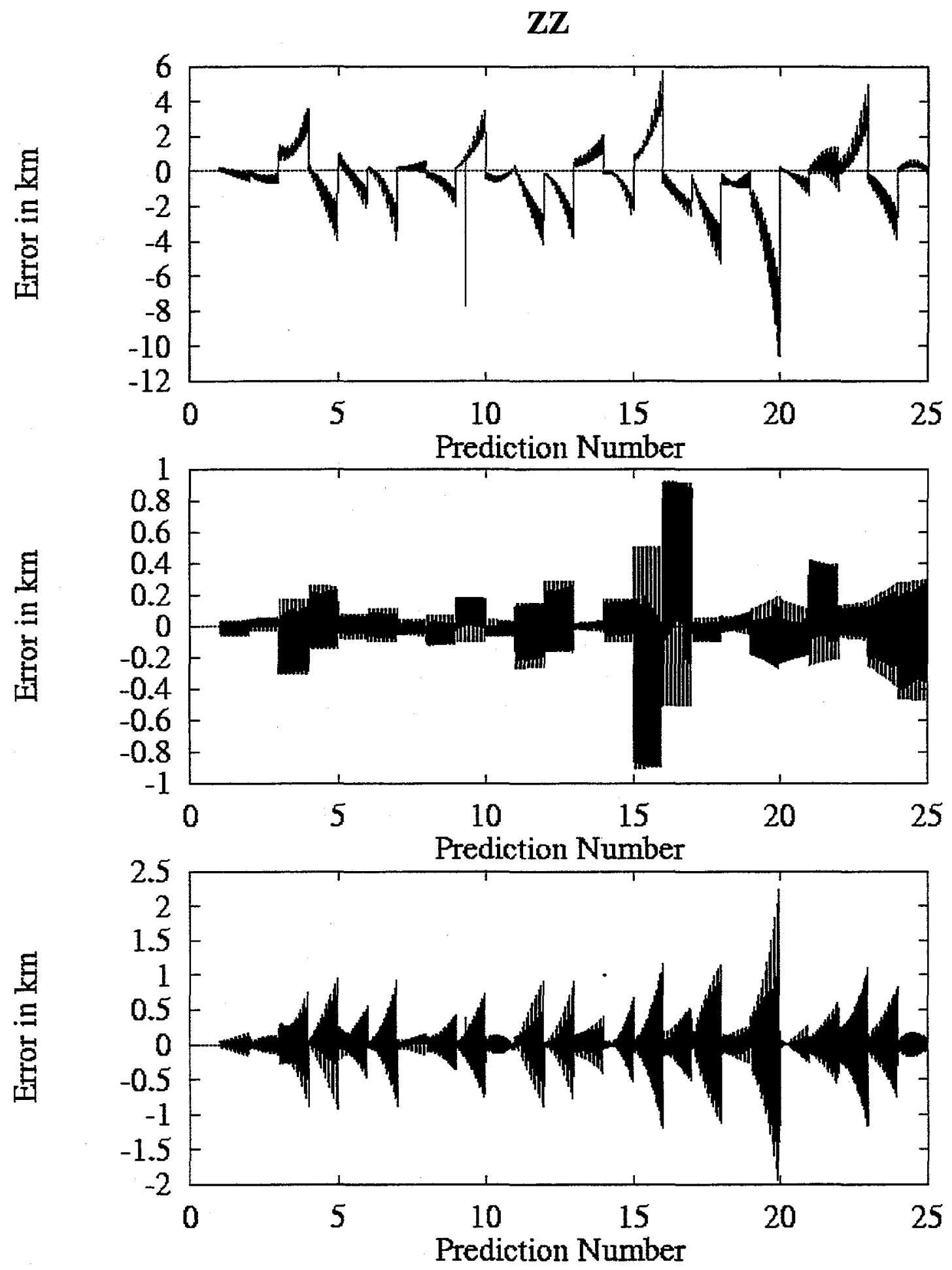

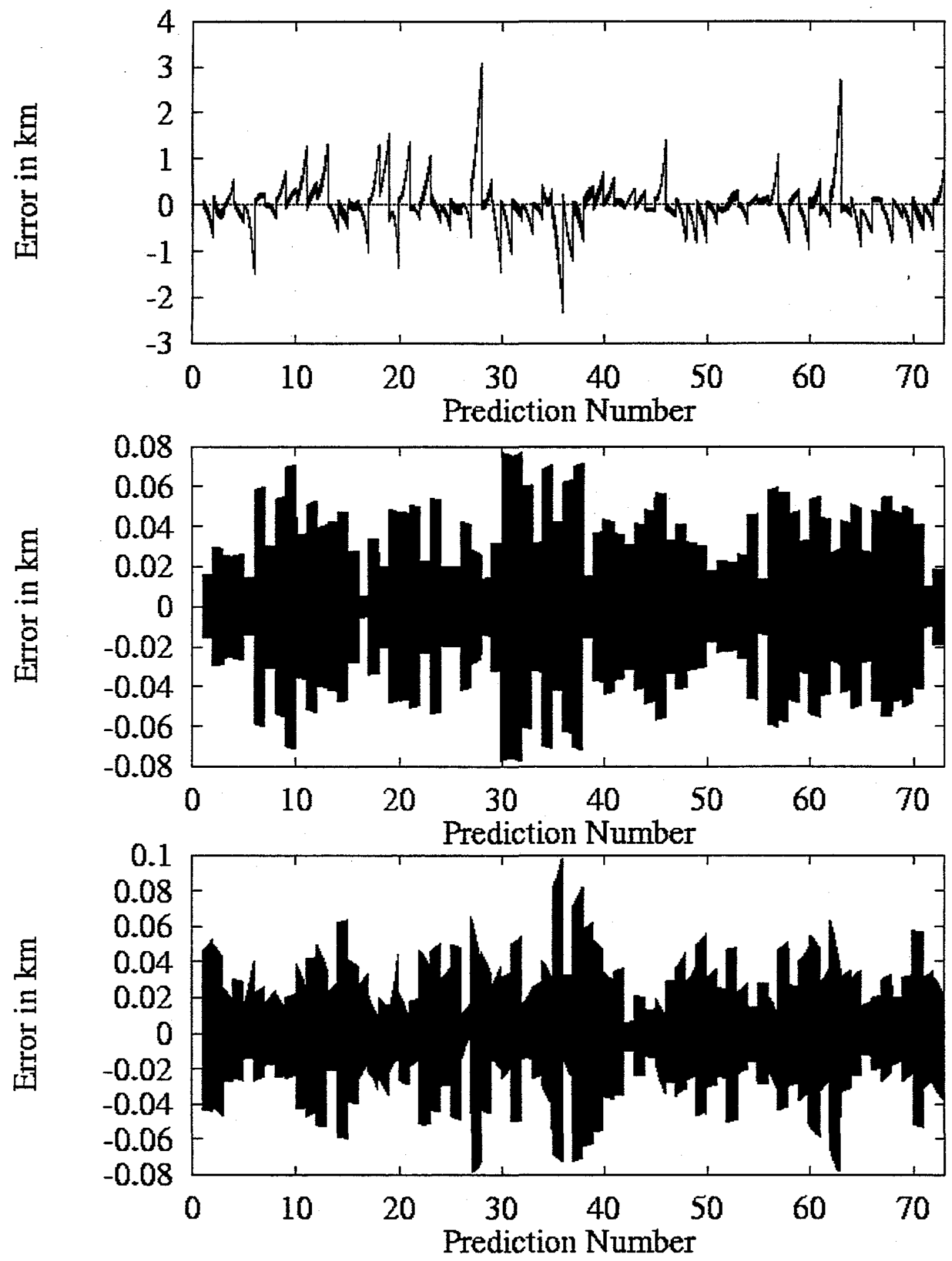
22
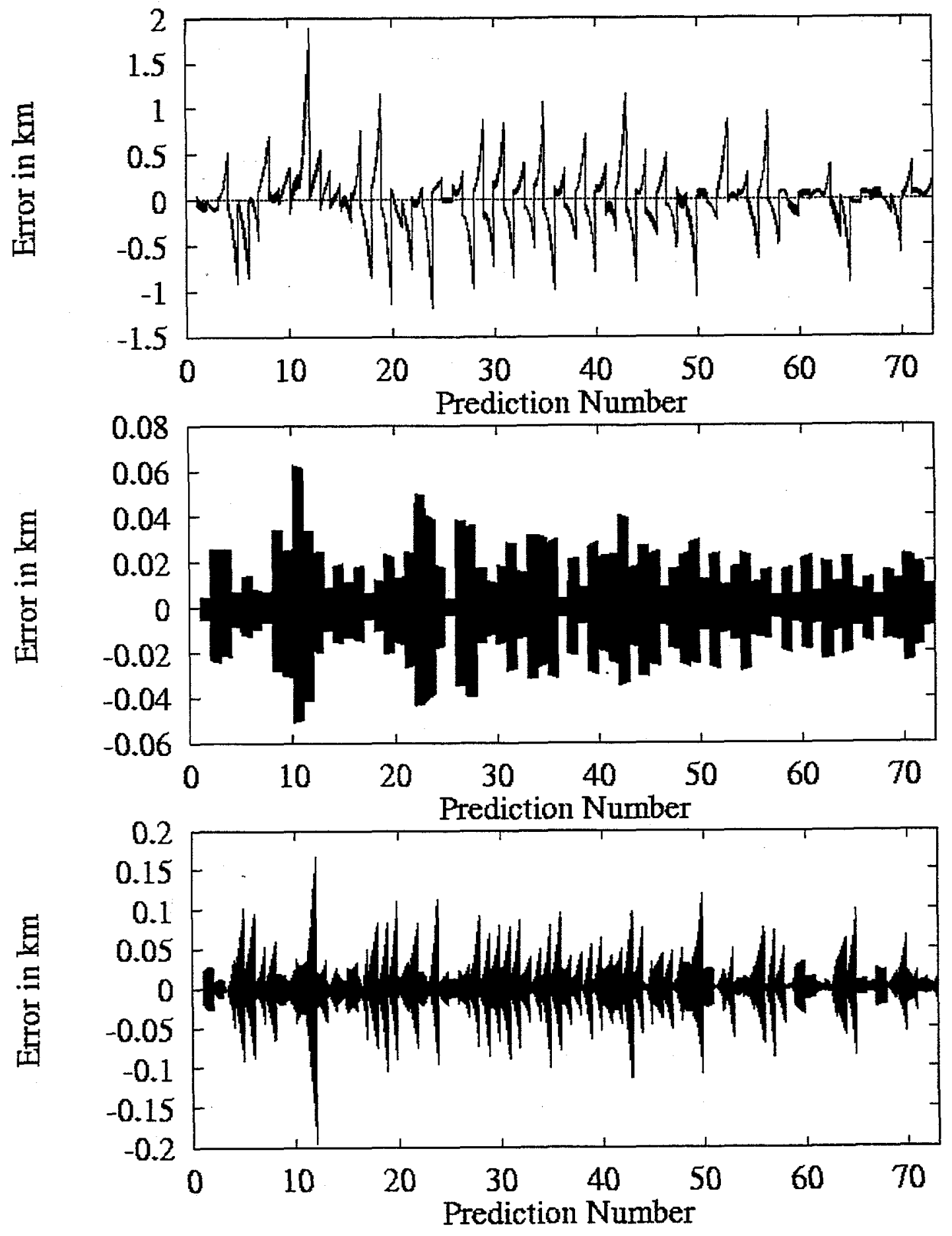
33

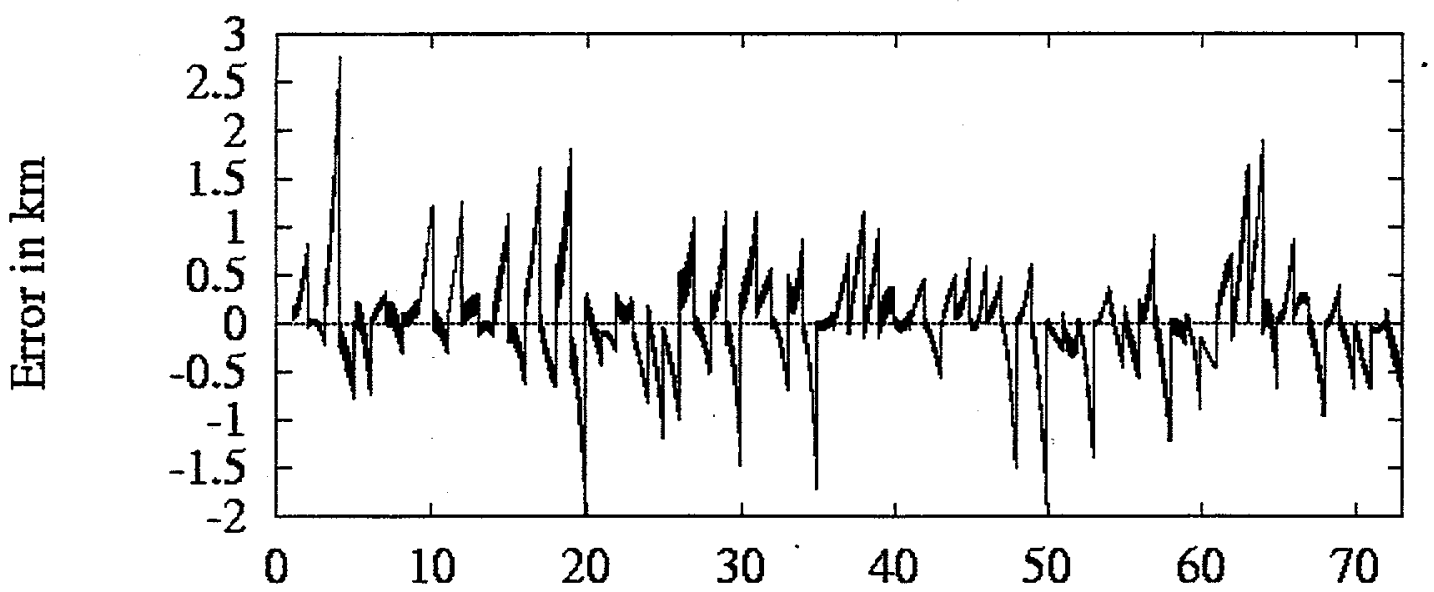

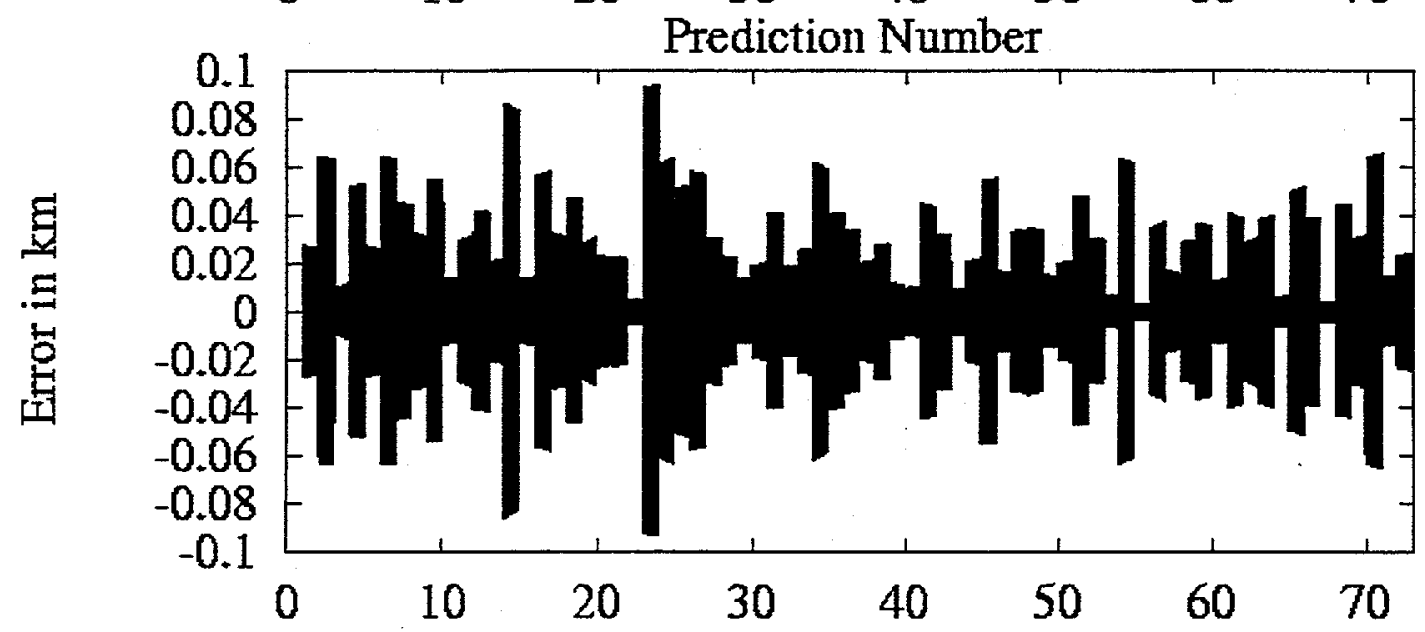

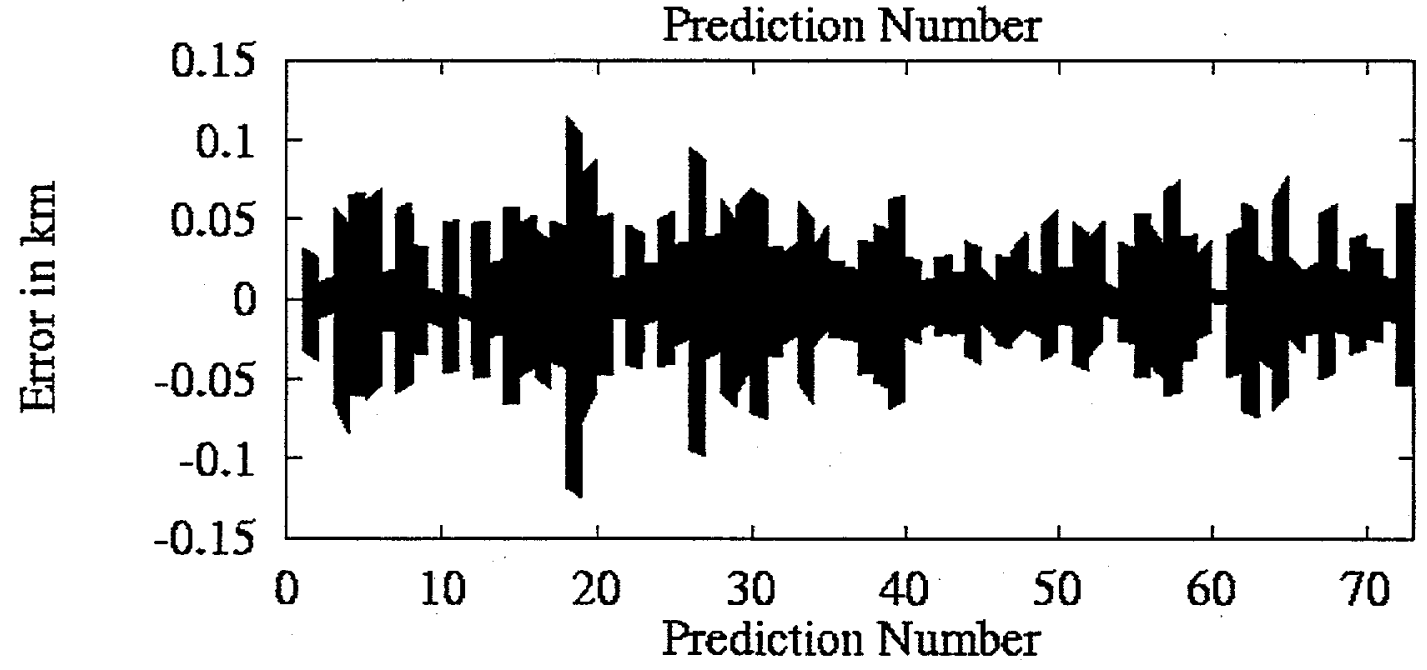


Appendix B:

RMS ERRORS AND 95 AND 99\% CONFIDENCE LIMITS 

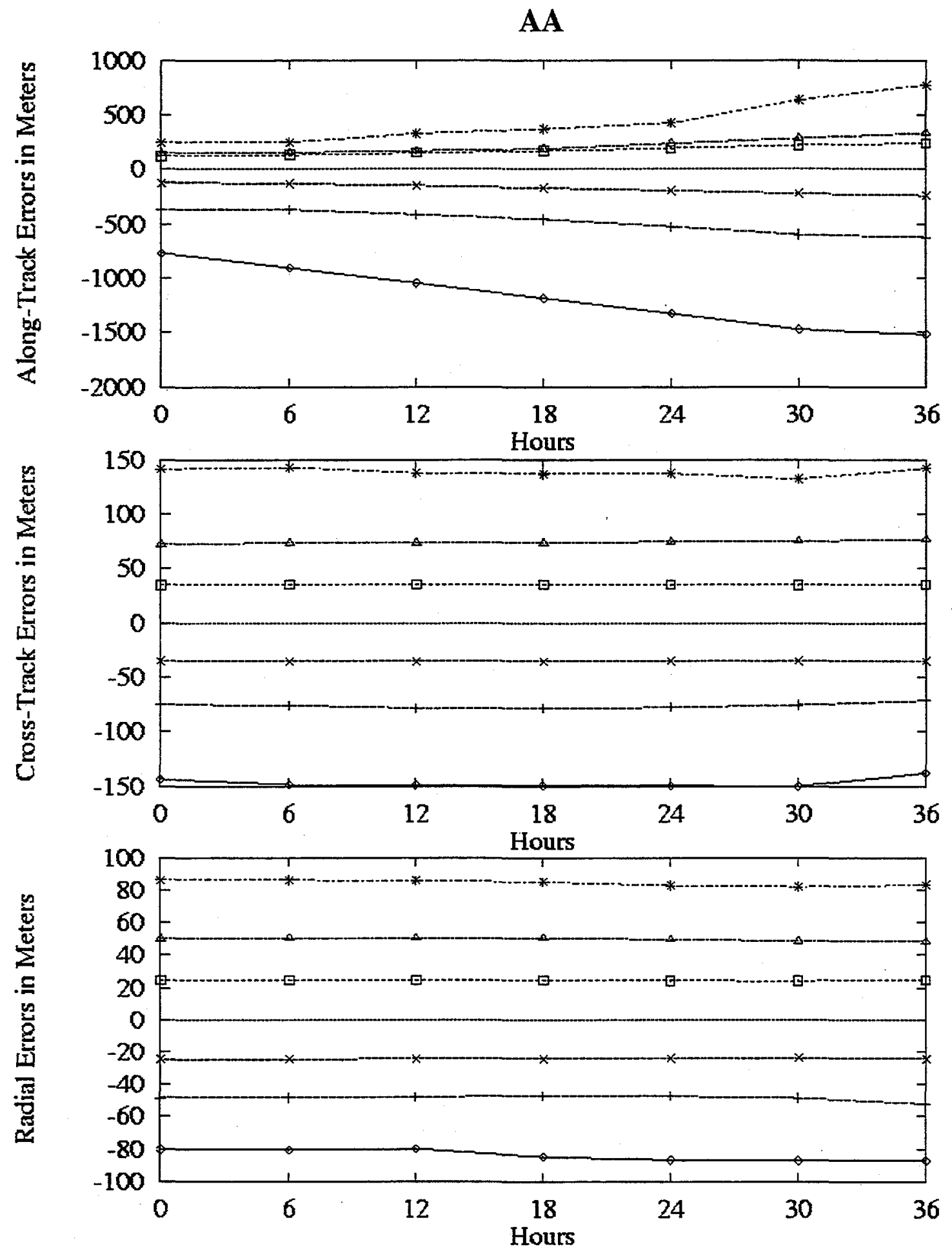


\section{BB}
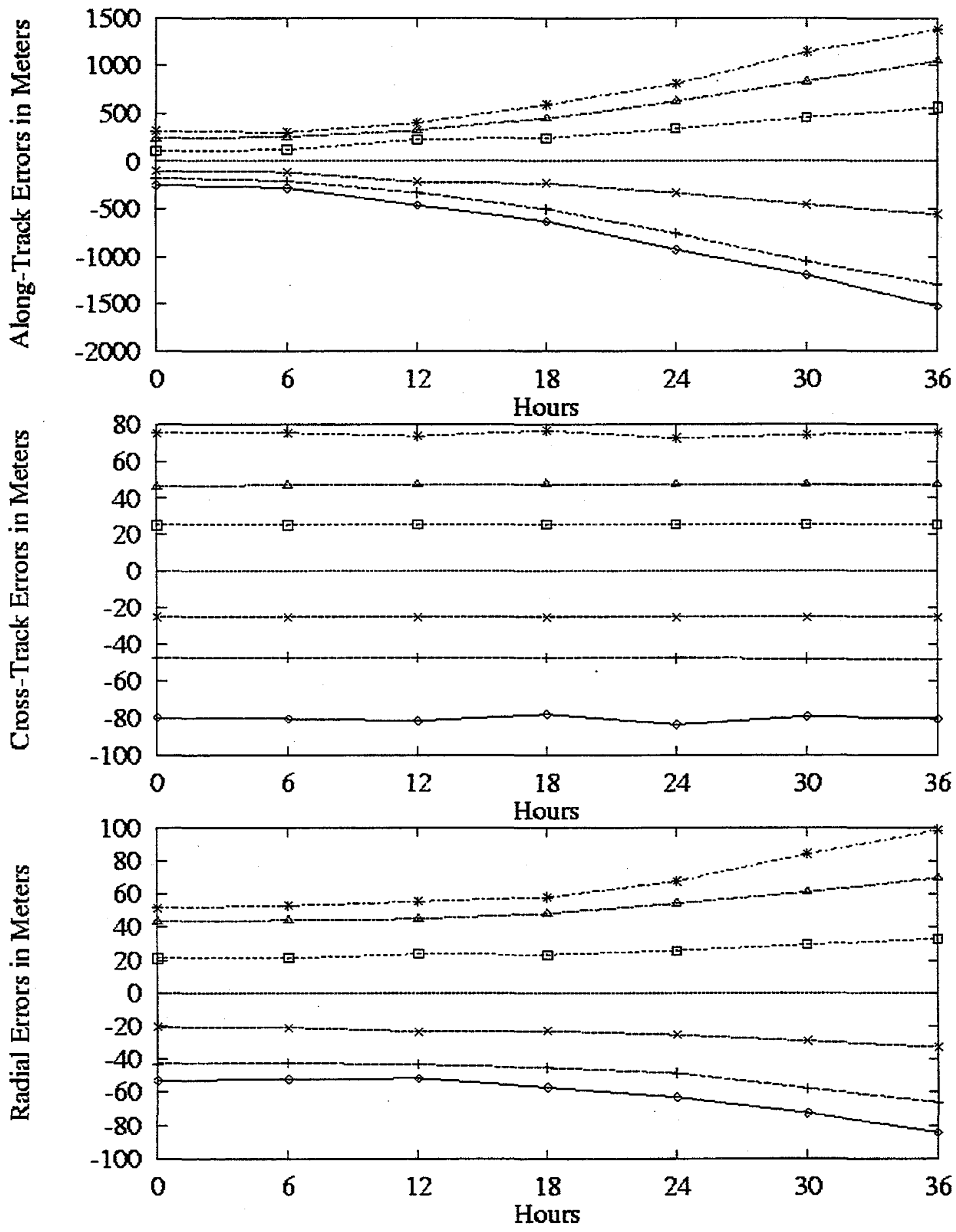
CC
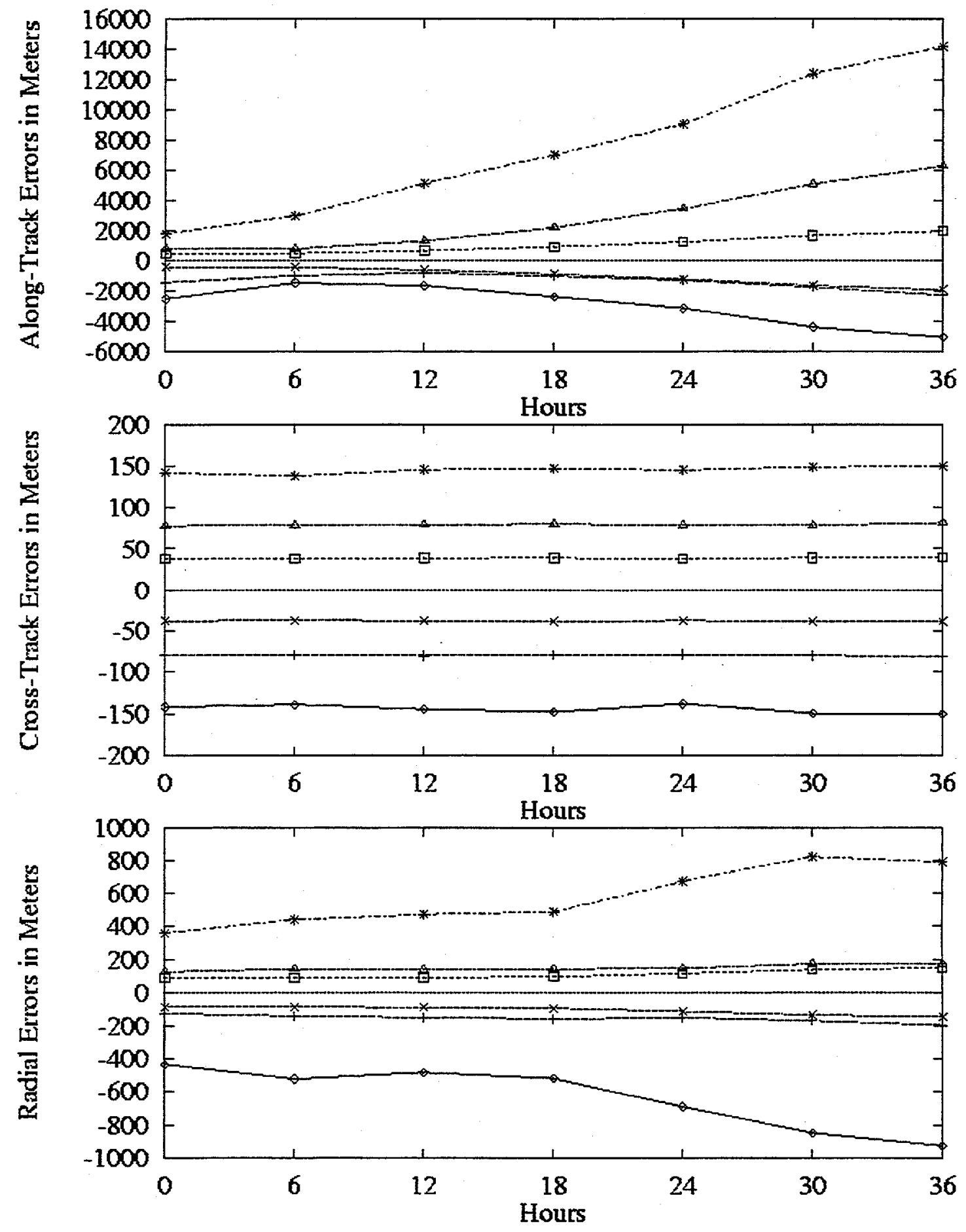
DD
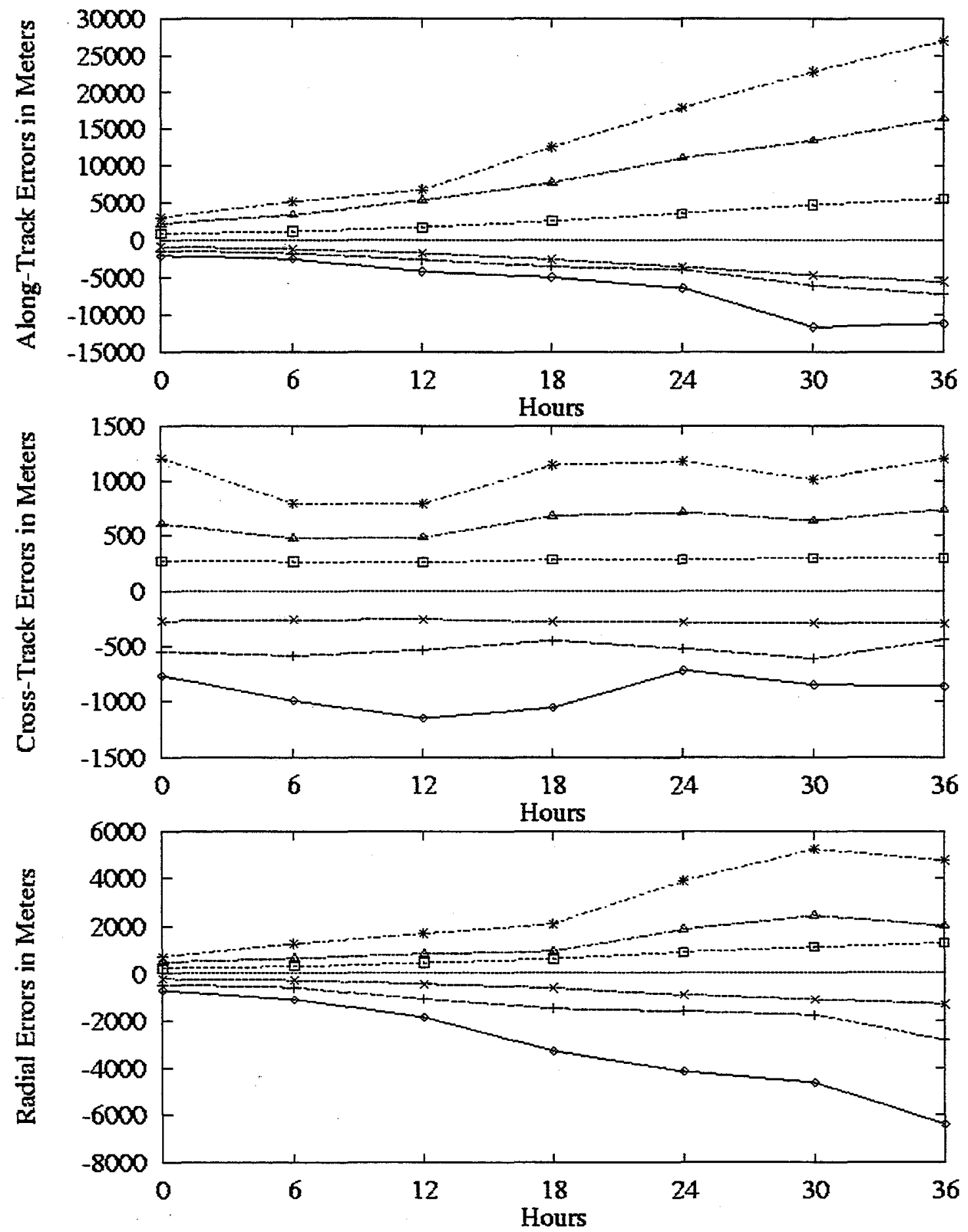
EE
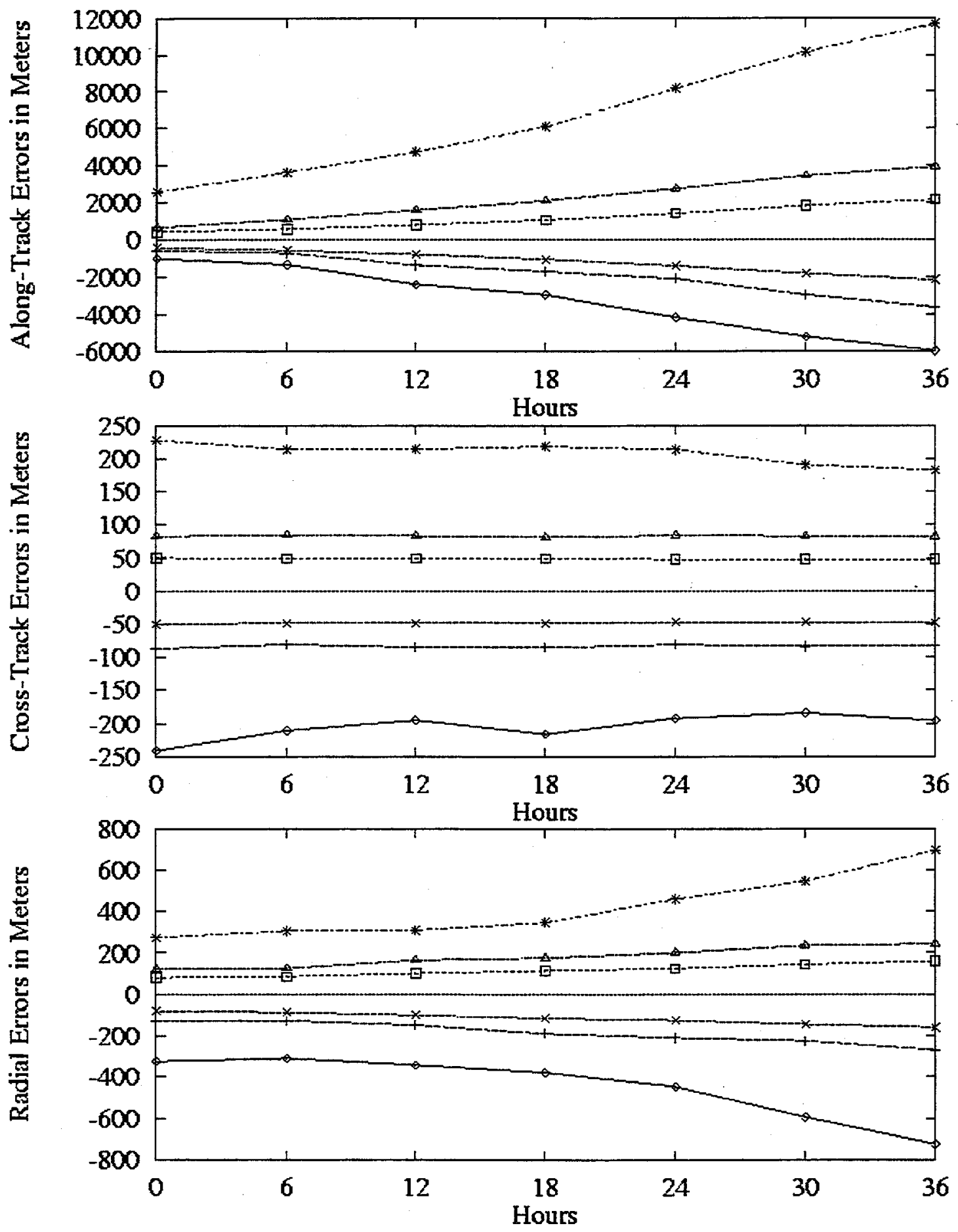

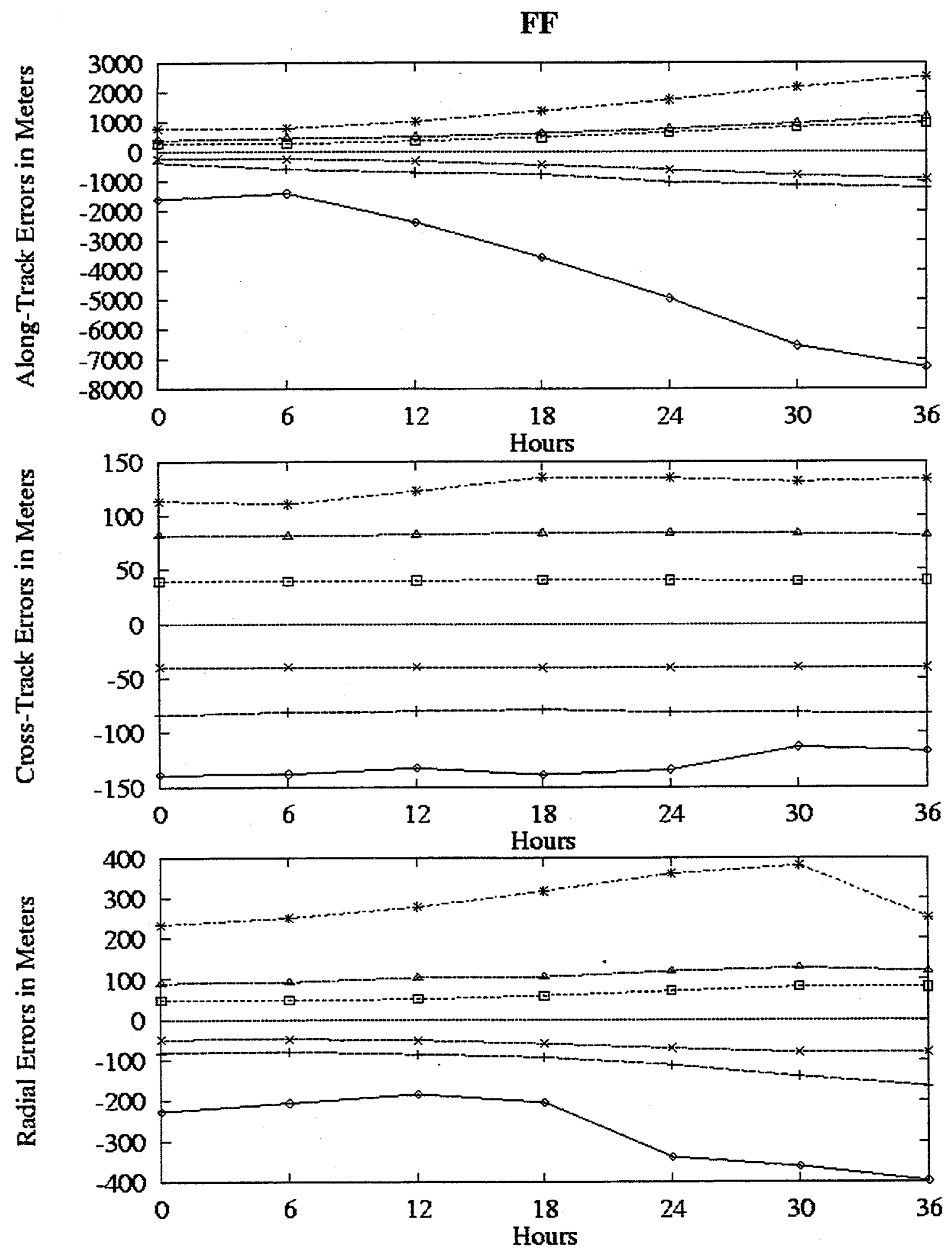


\section{GG}
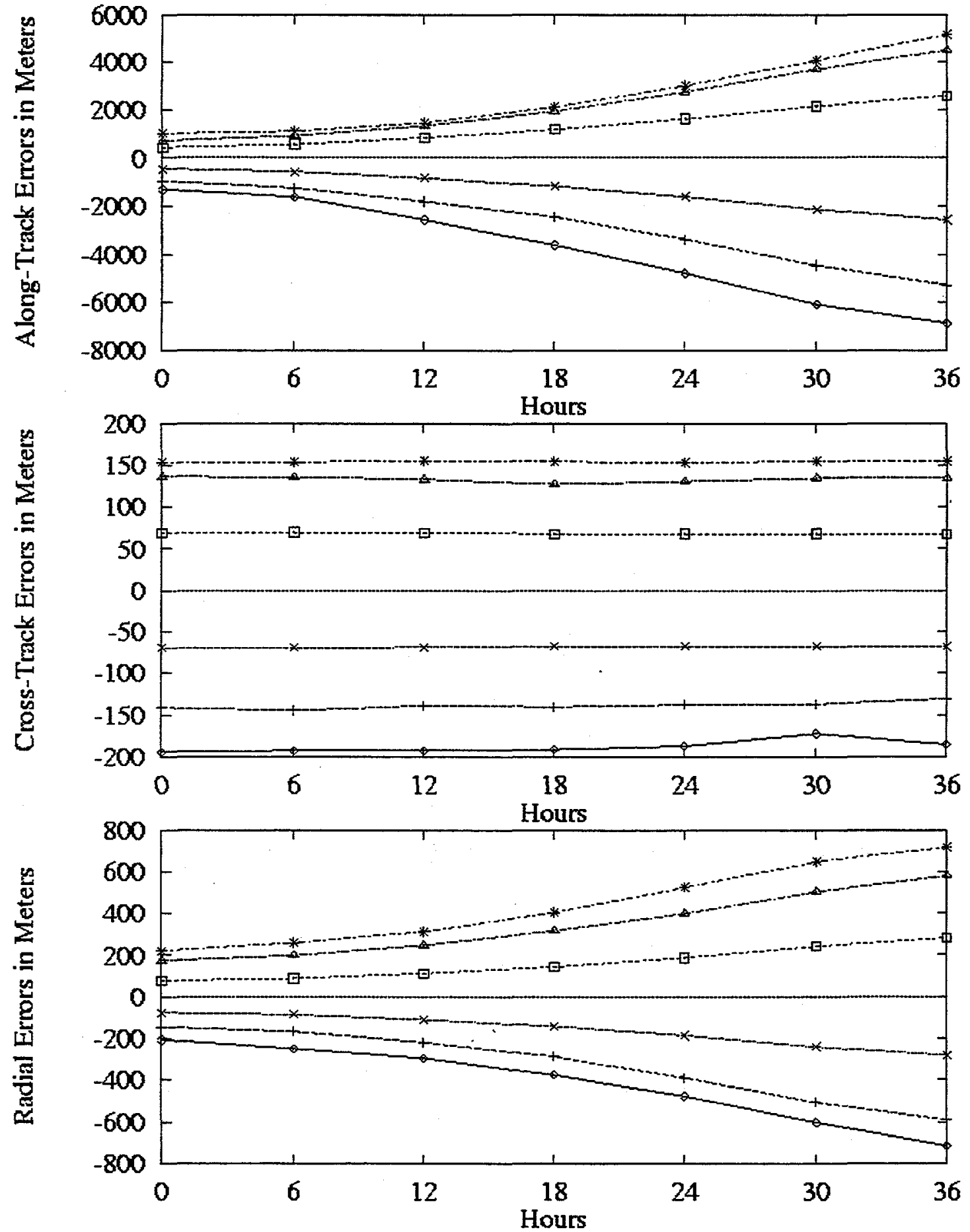


\section{HH}
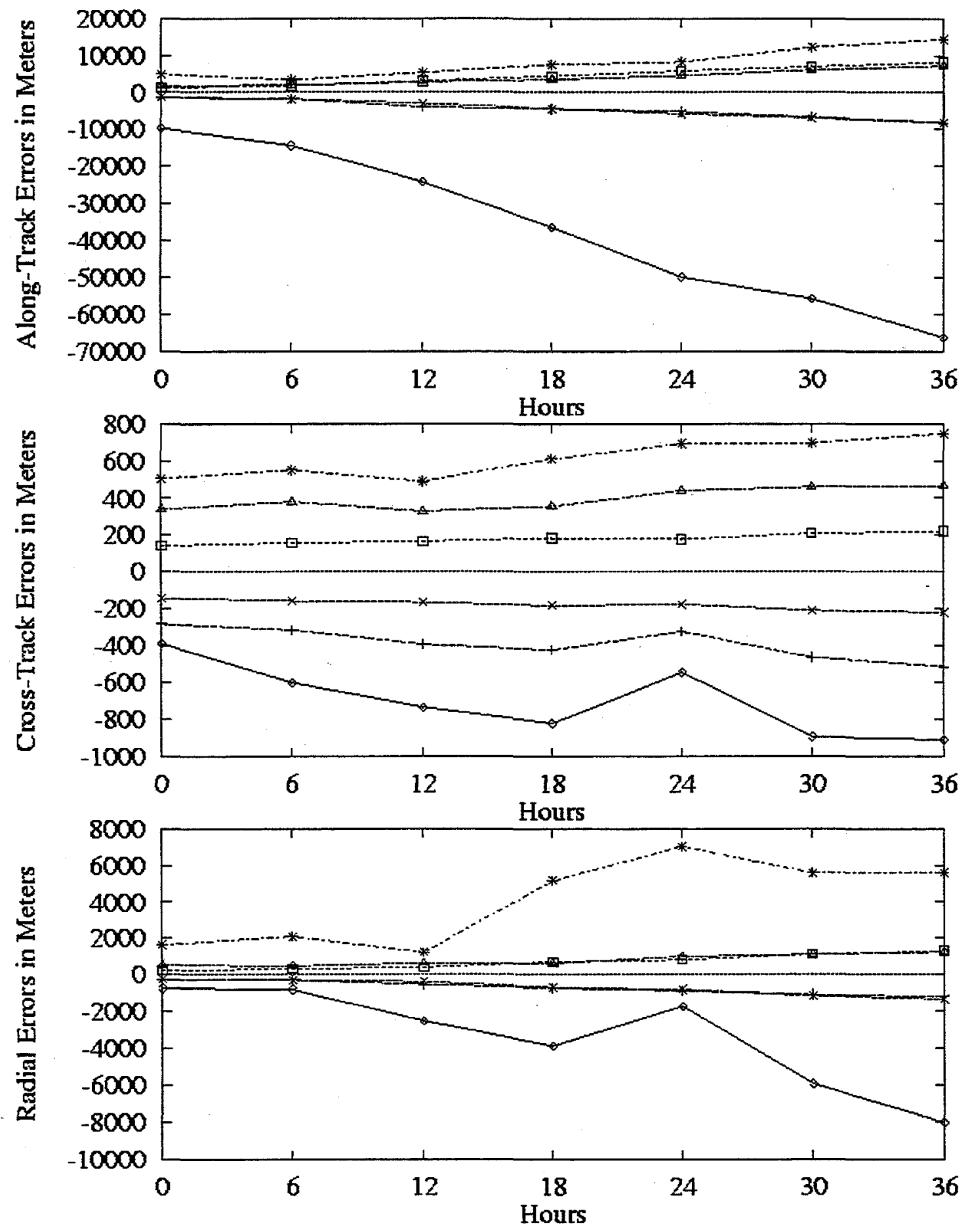

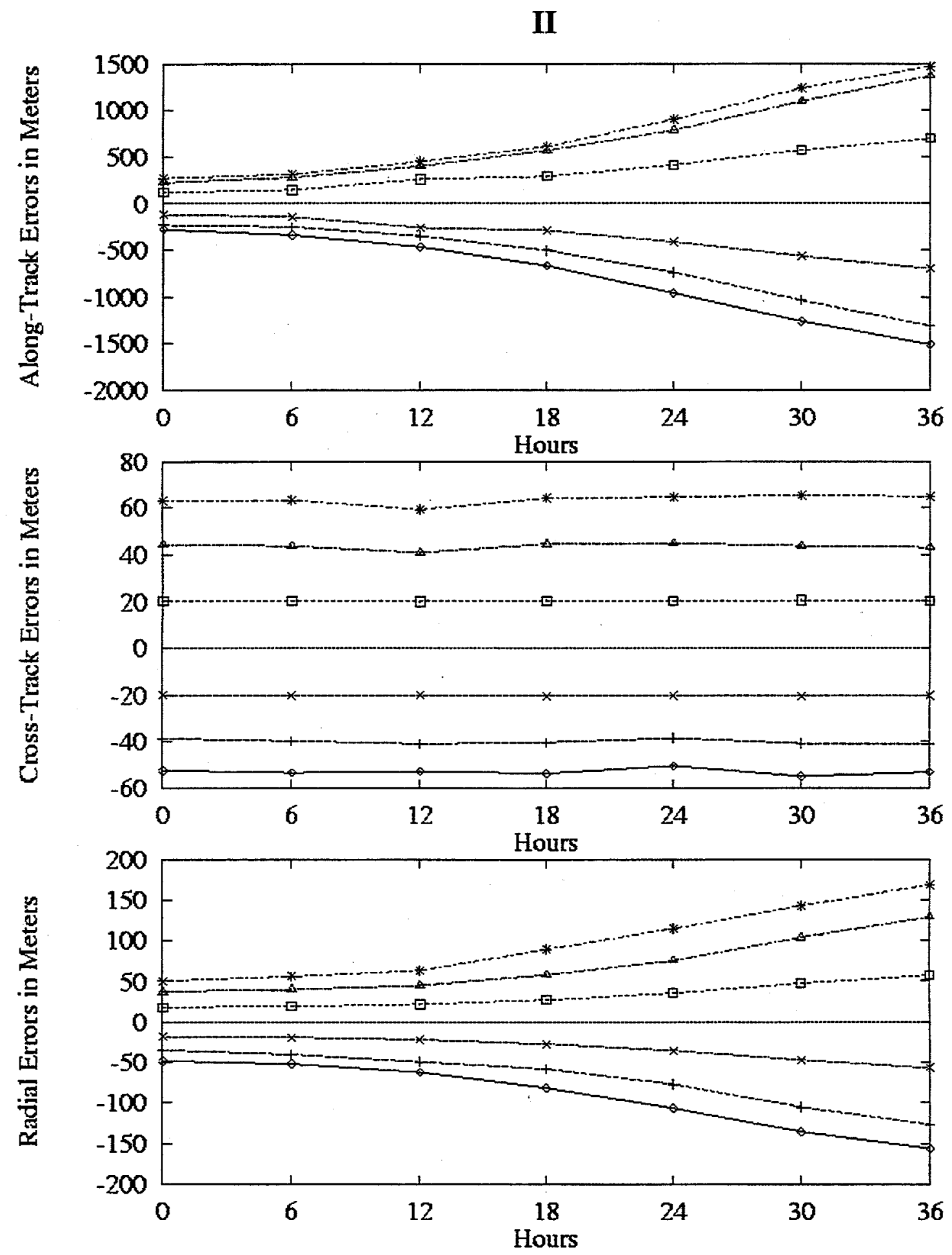

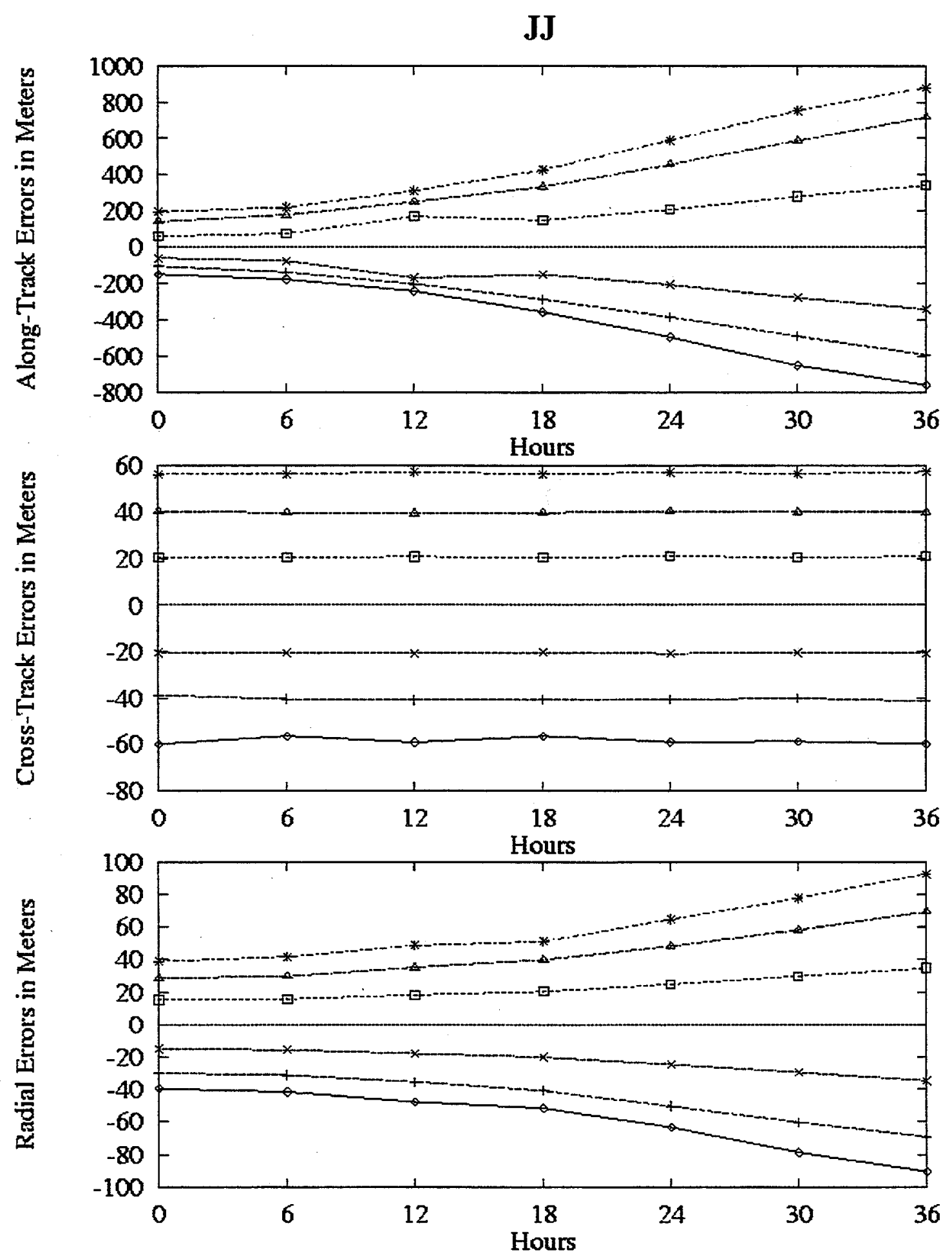
KK
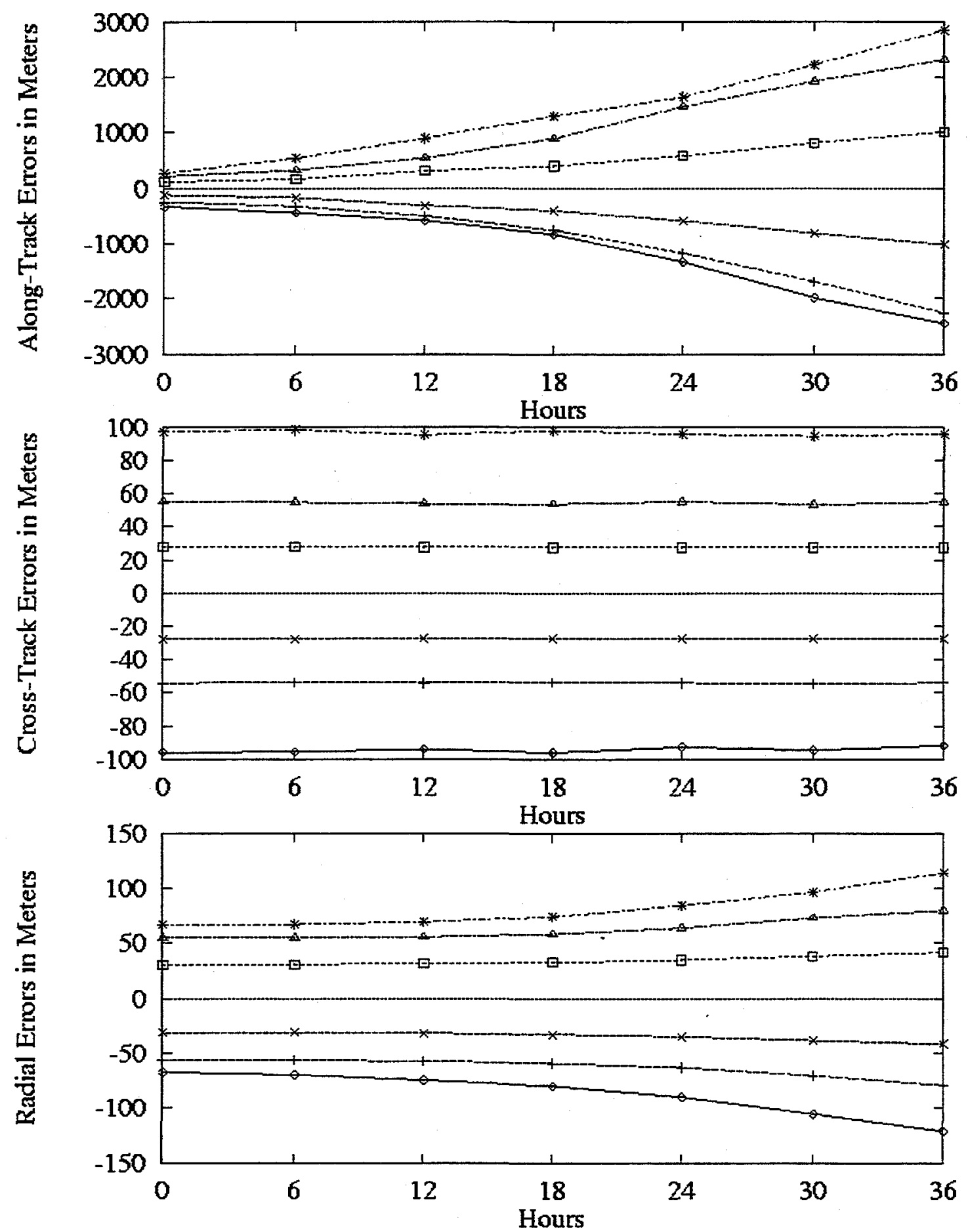
LL
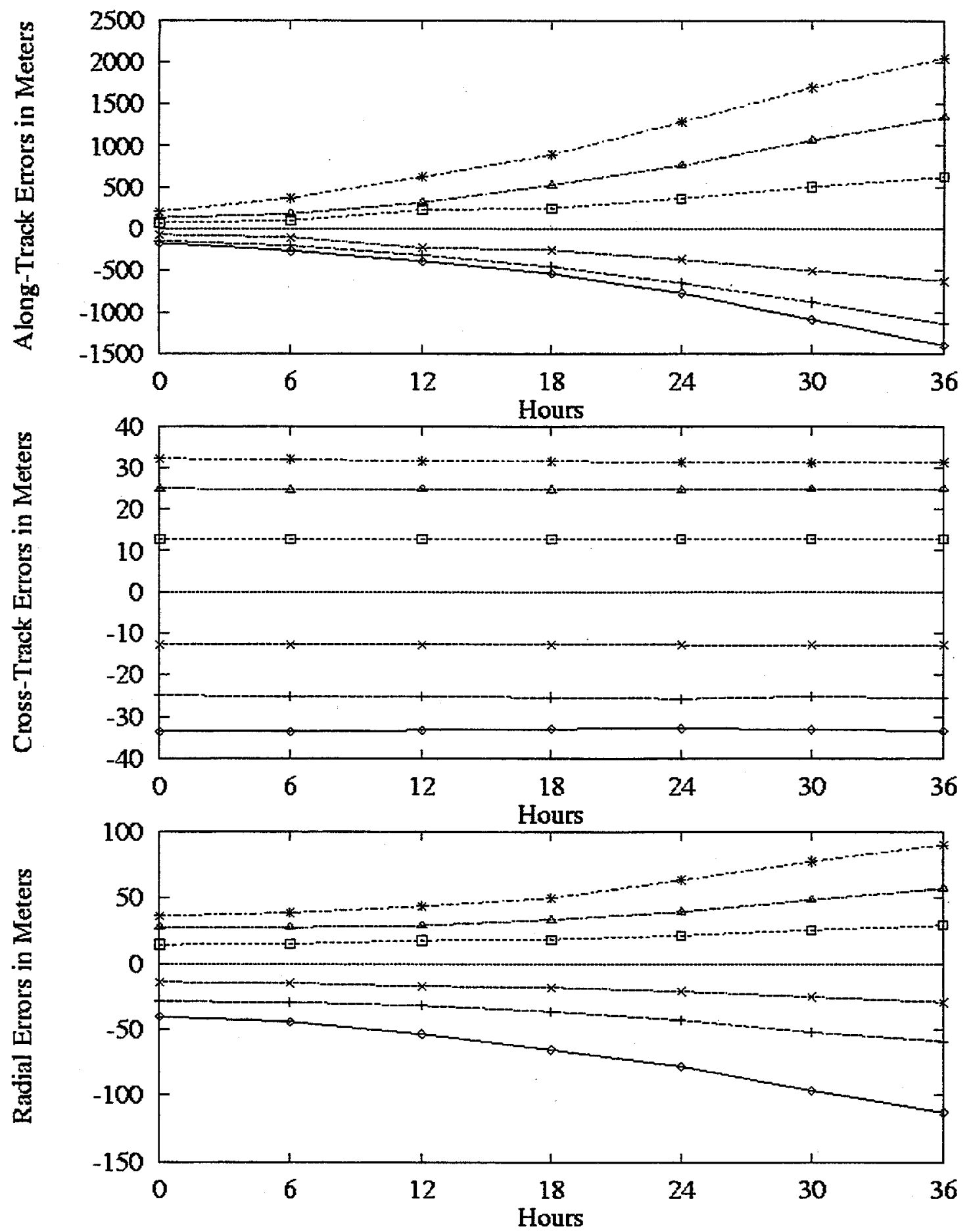


\section{MM}
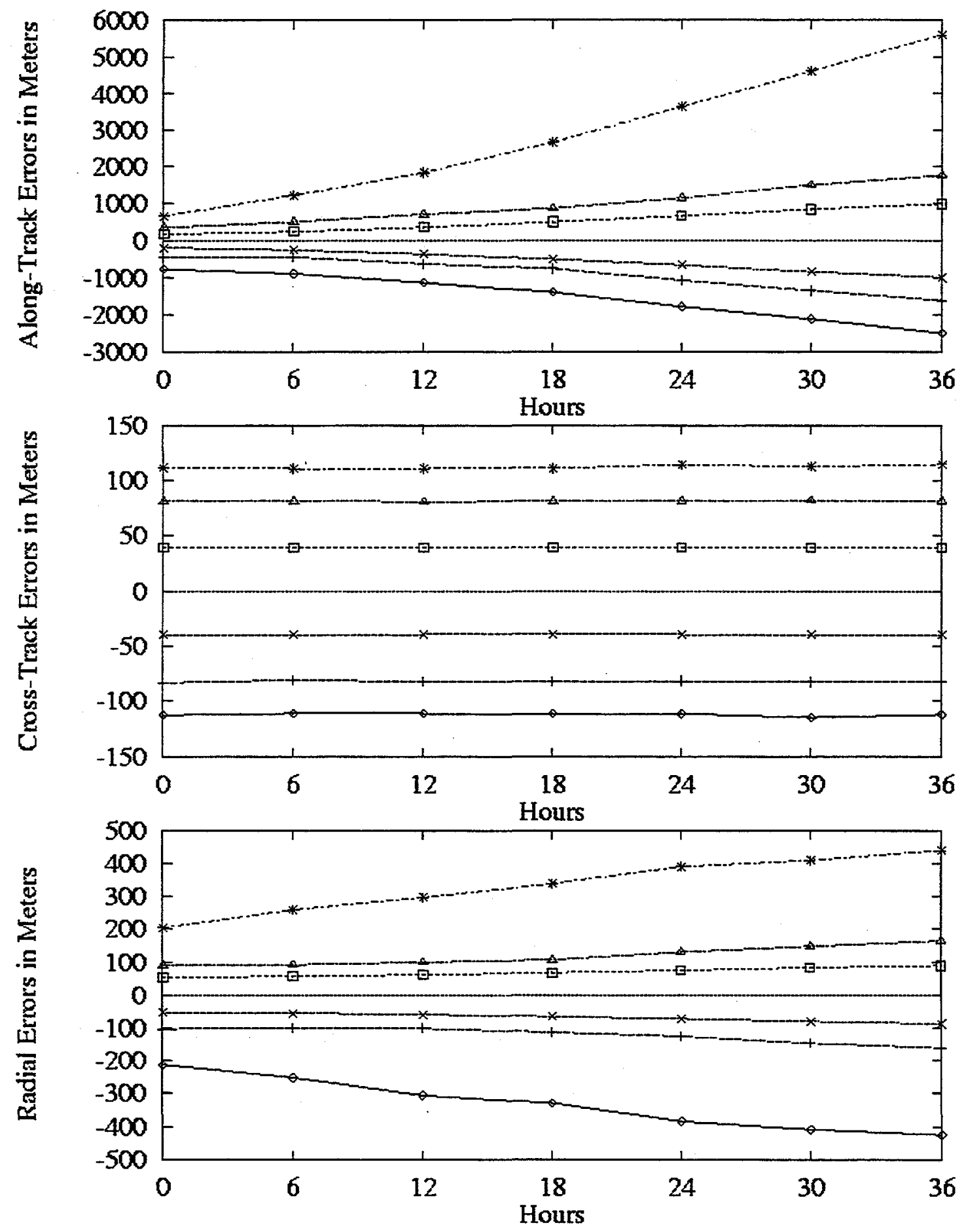

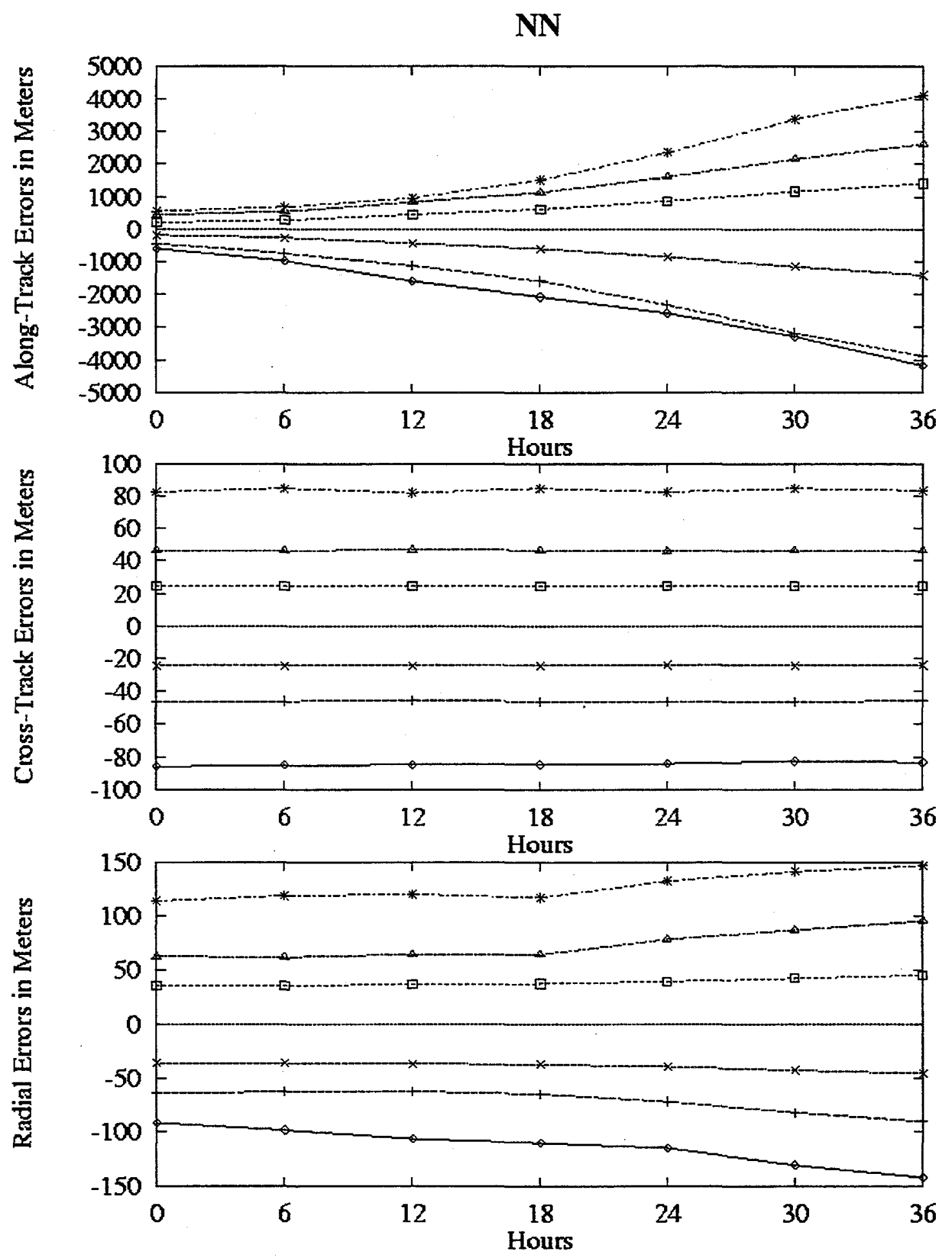

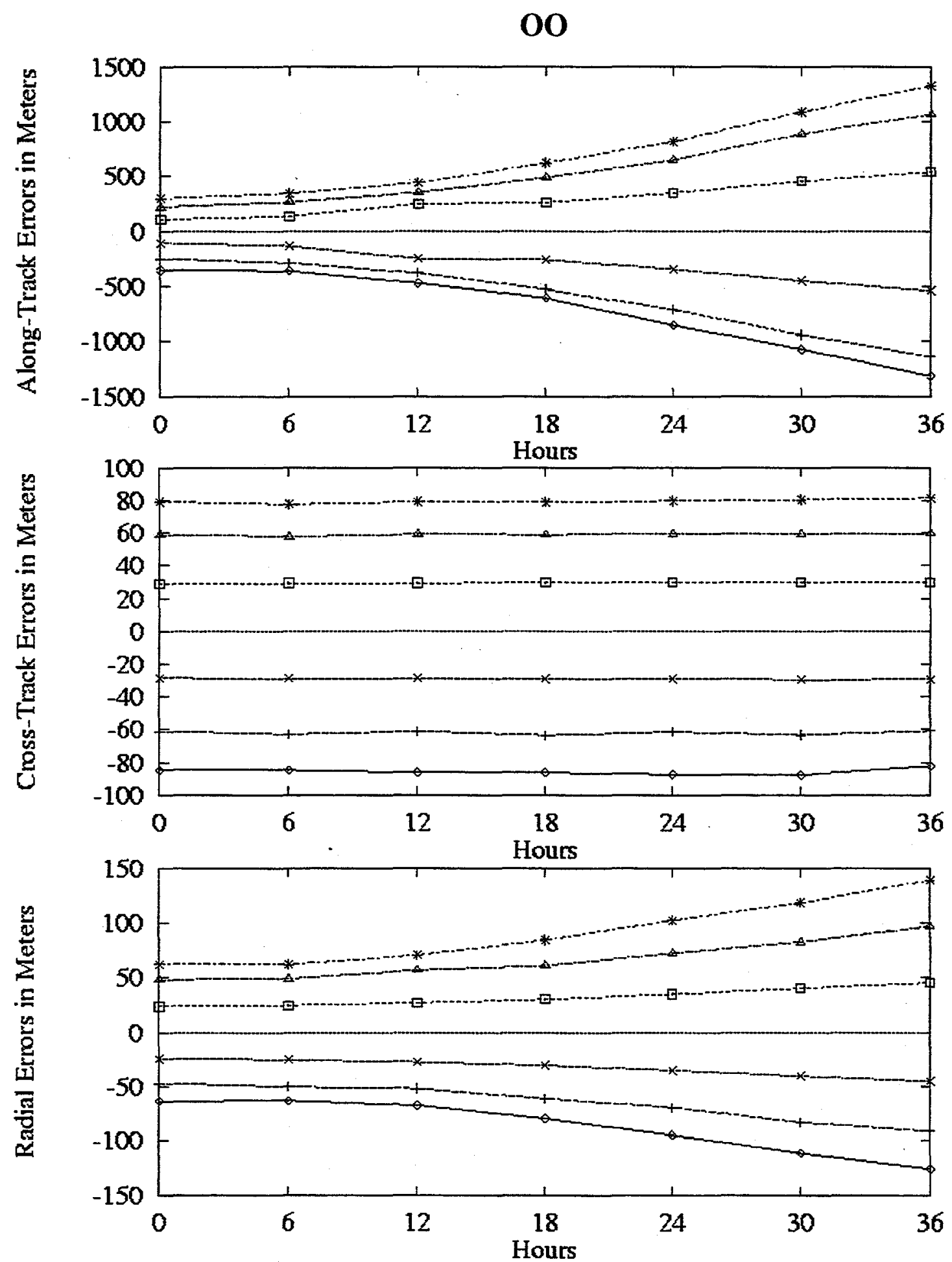

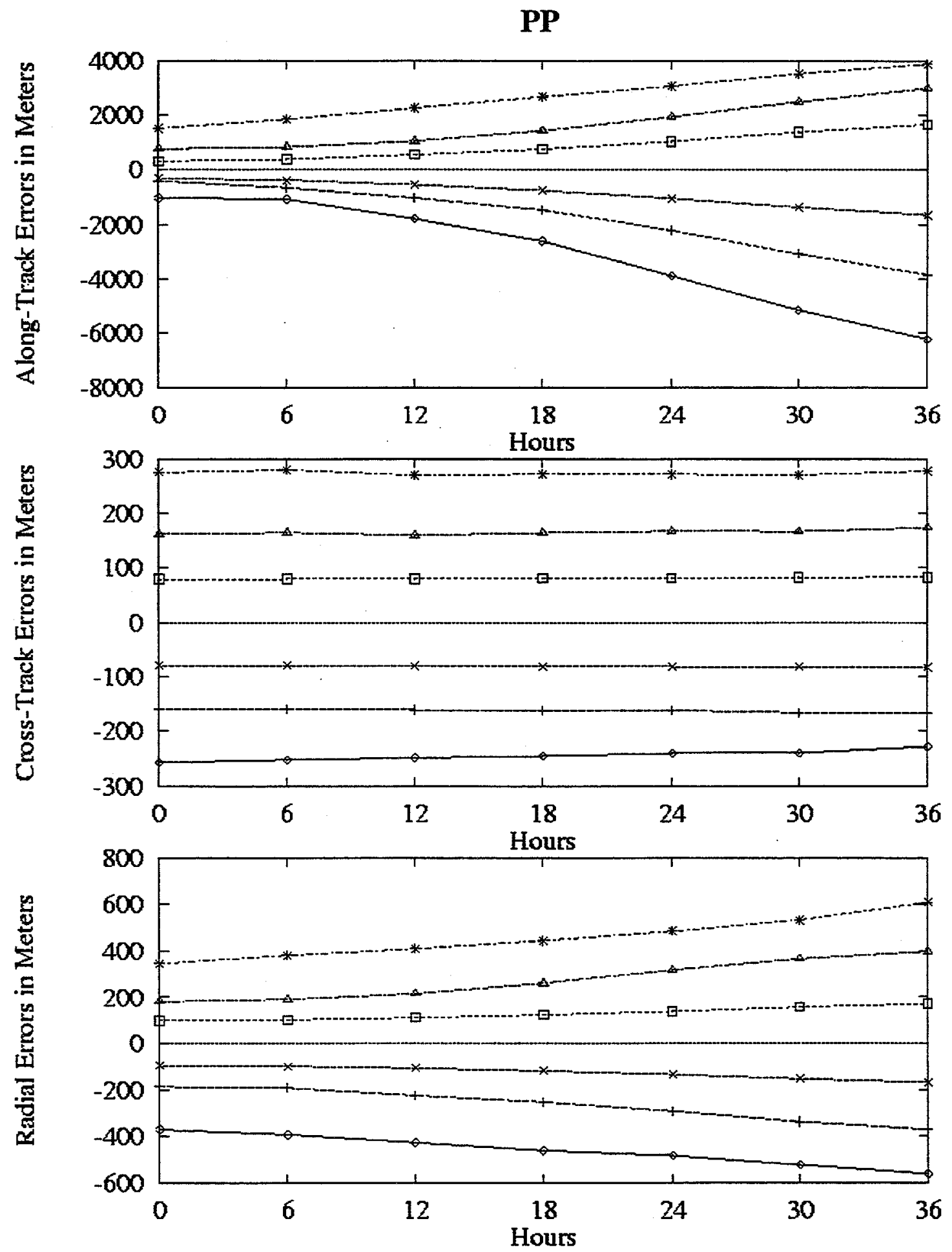
QQ
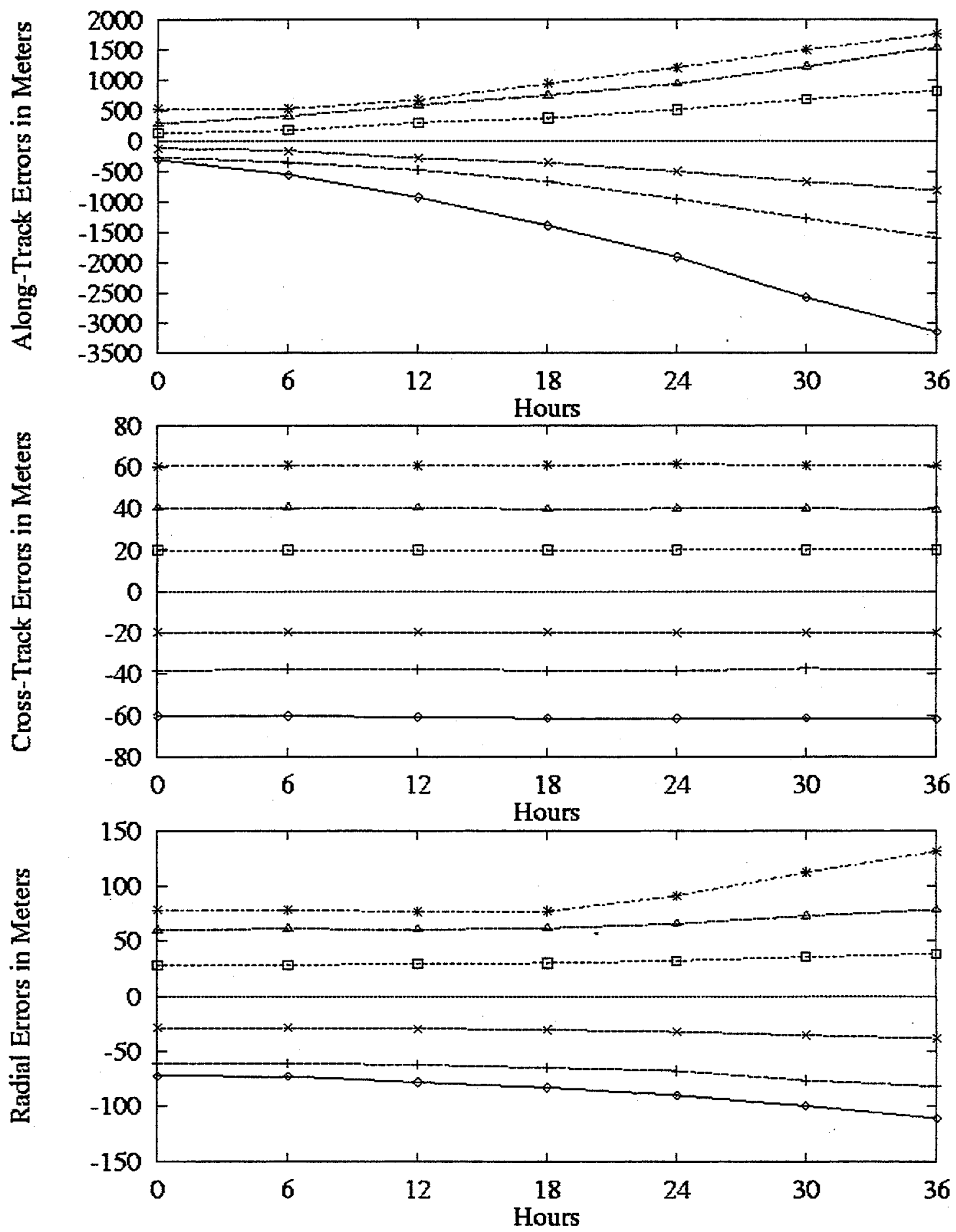


\section{RR}
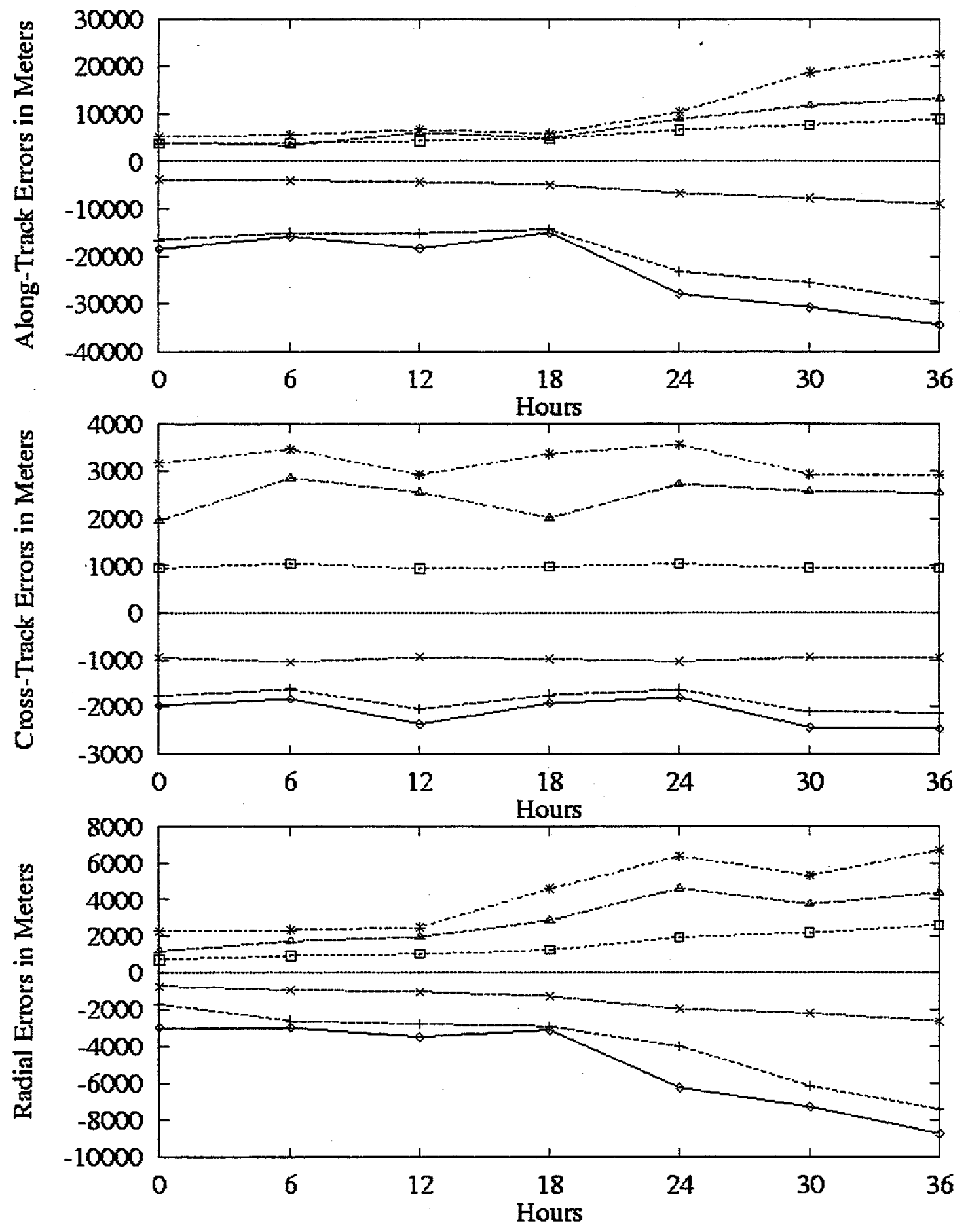

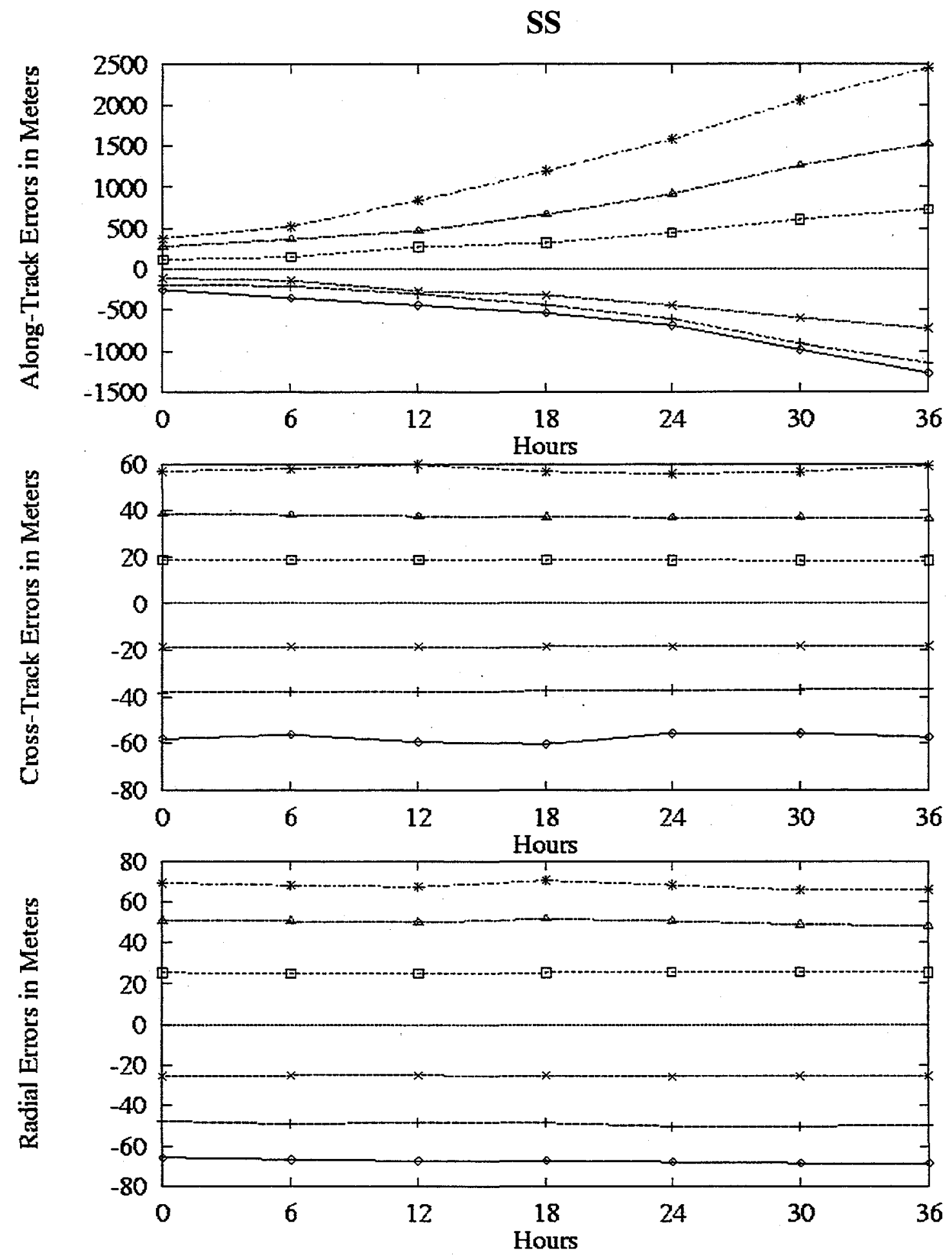
TT
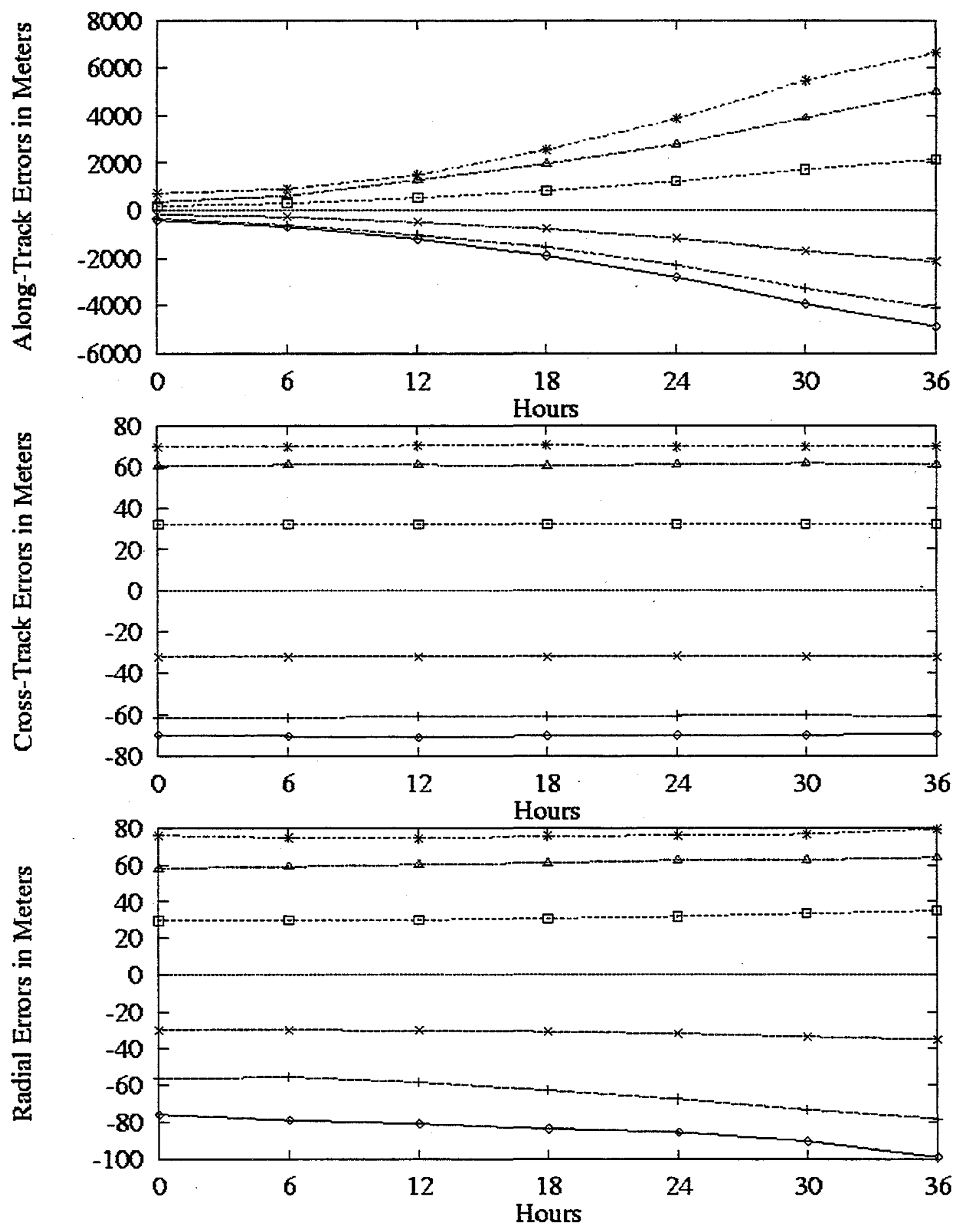


\section{UU}
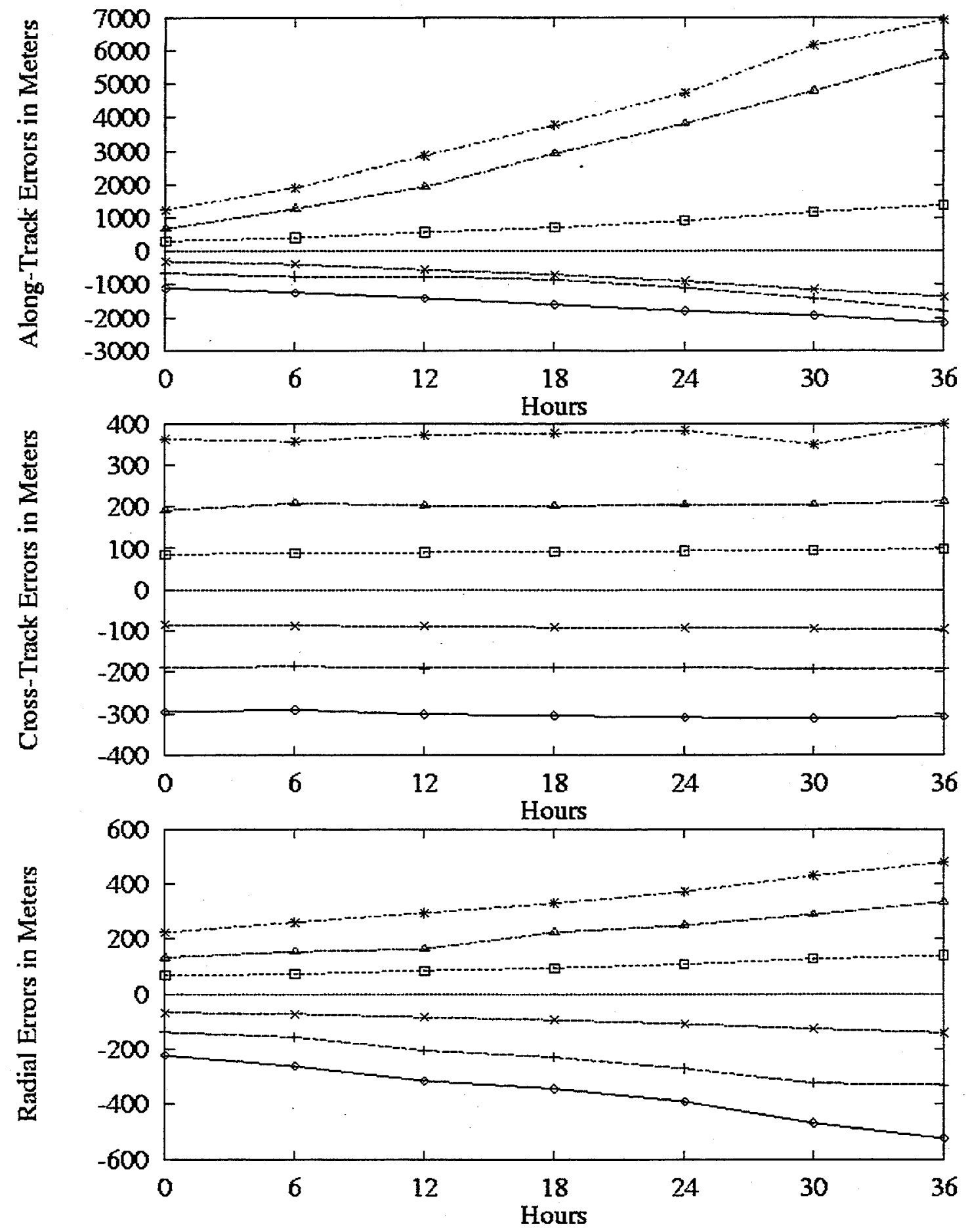

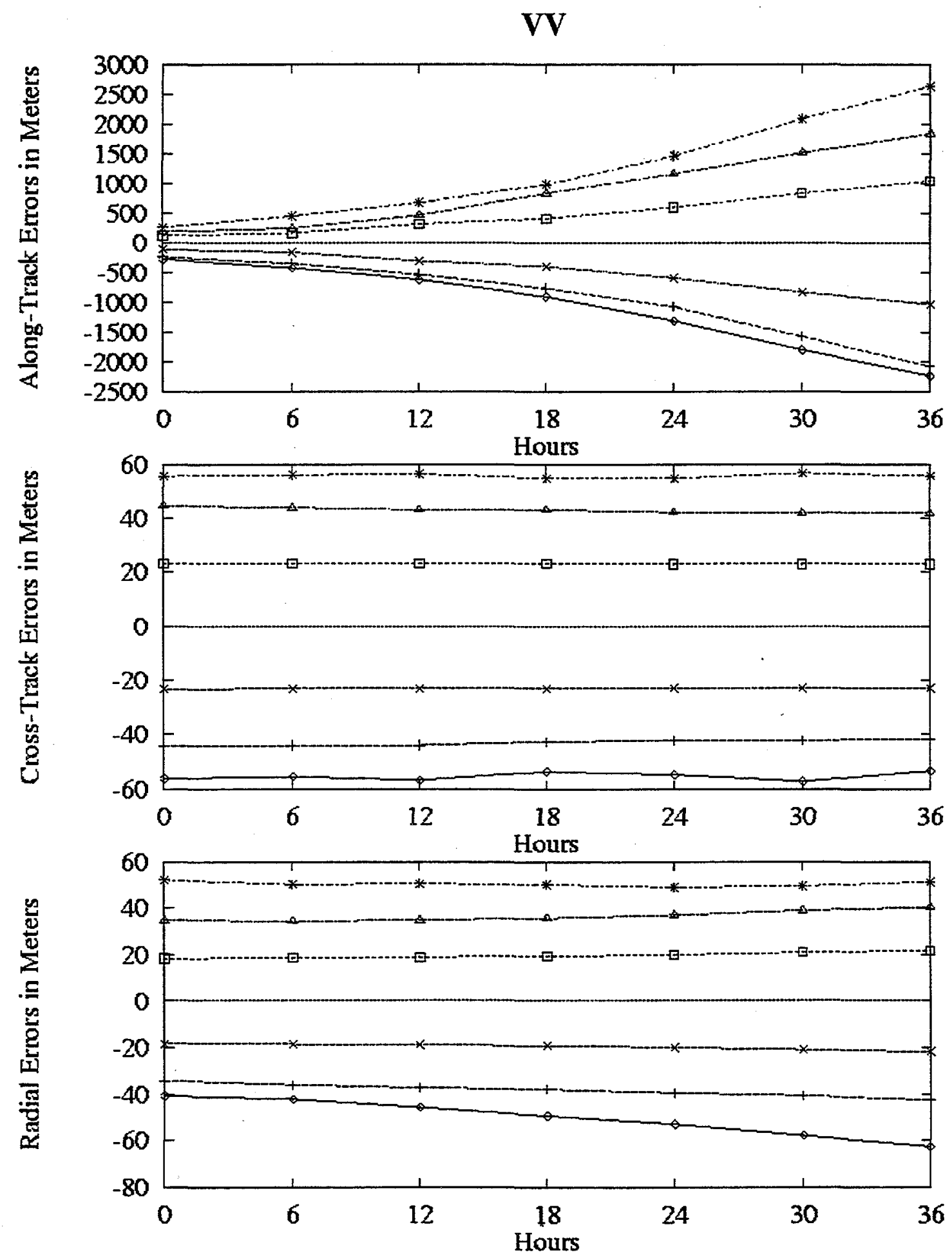

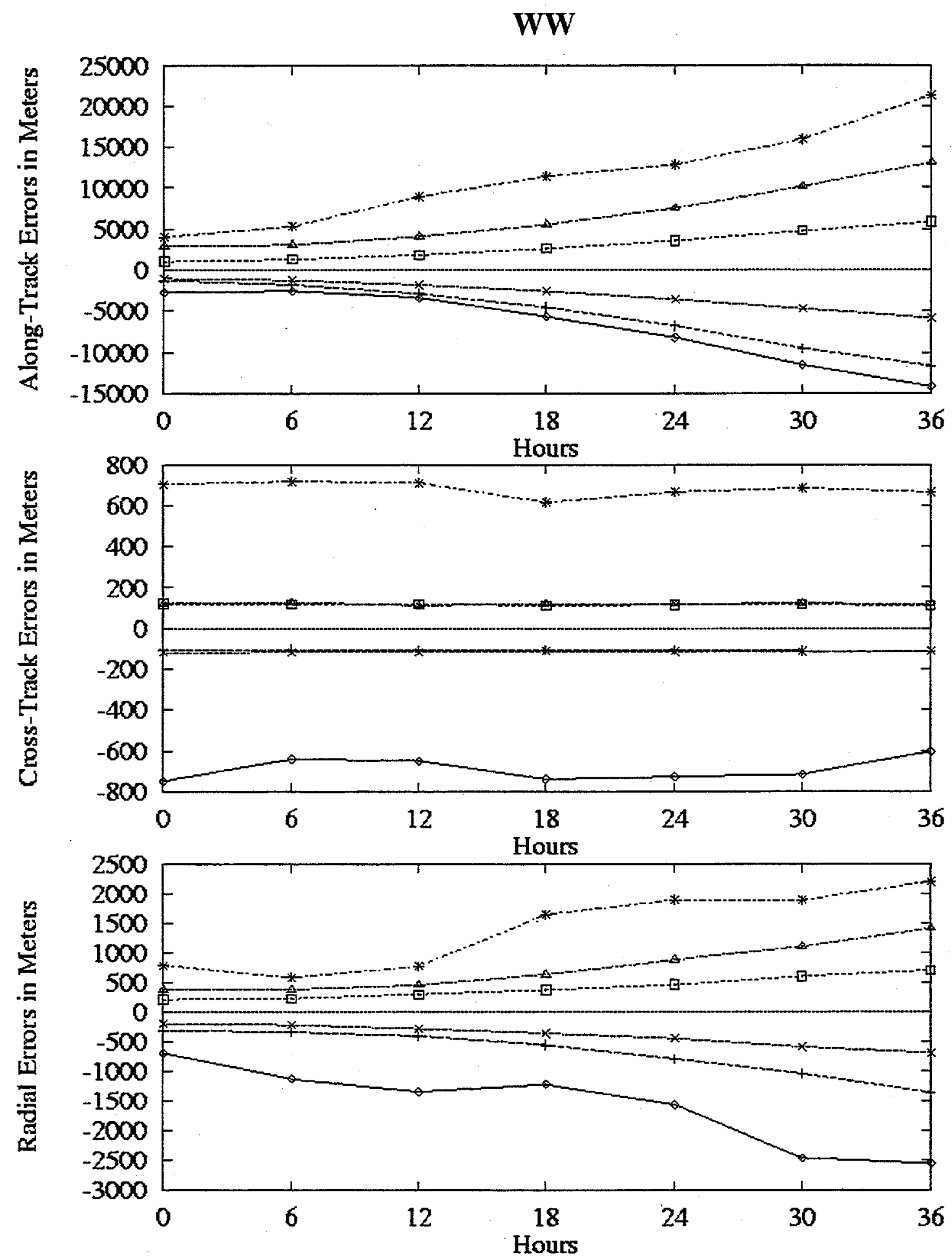


\section{XX}
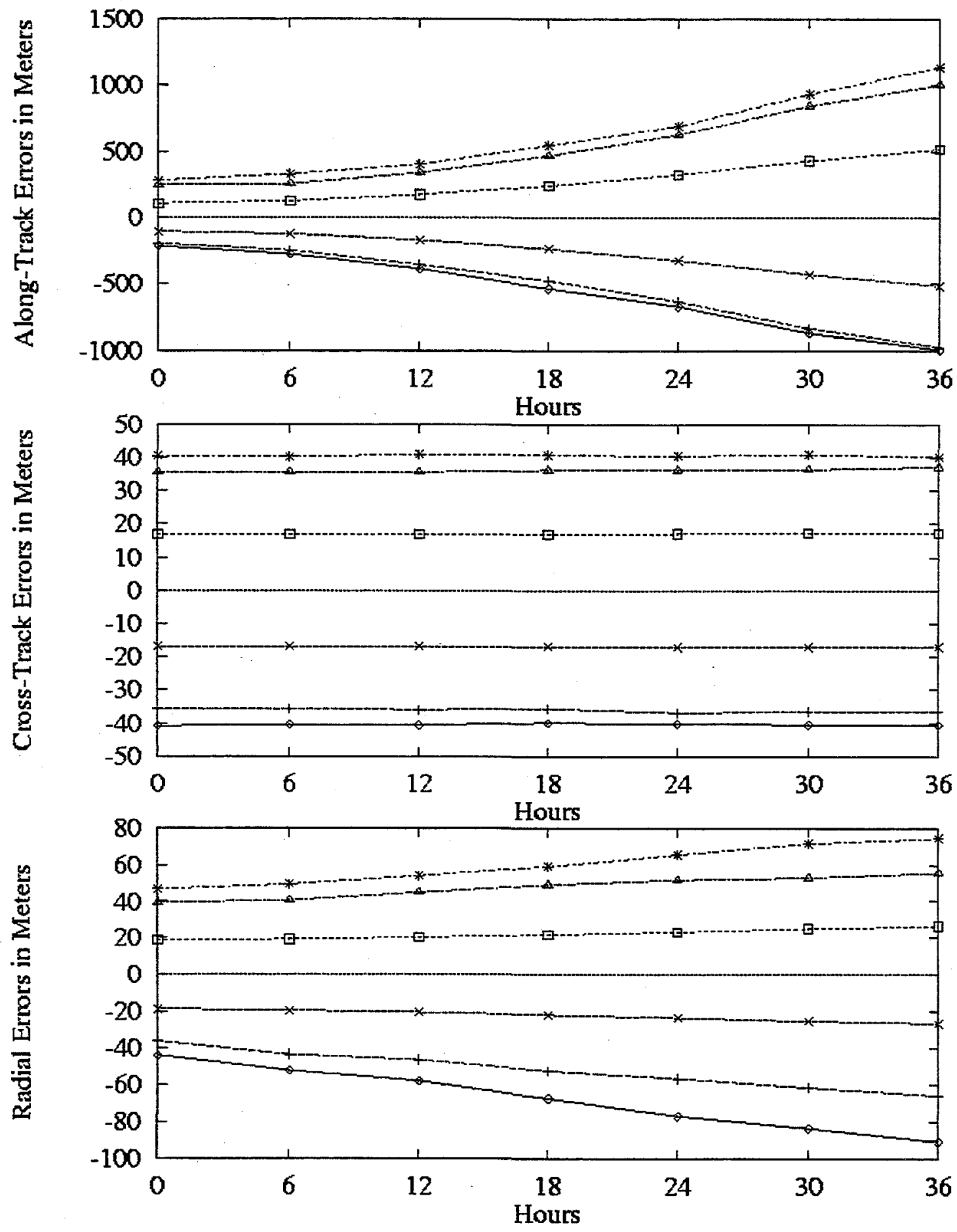

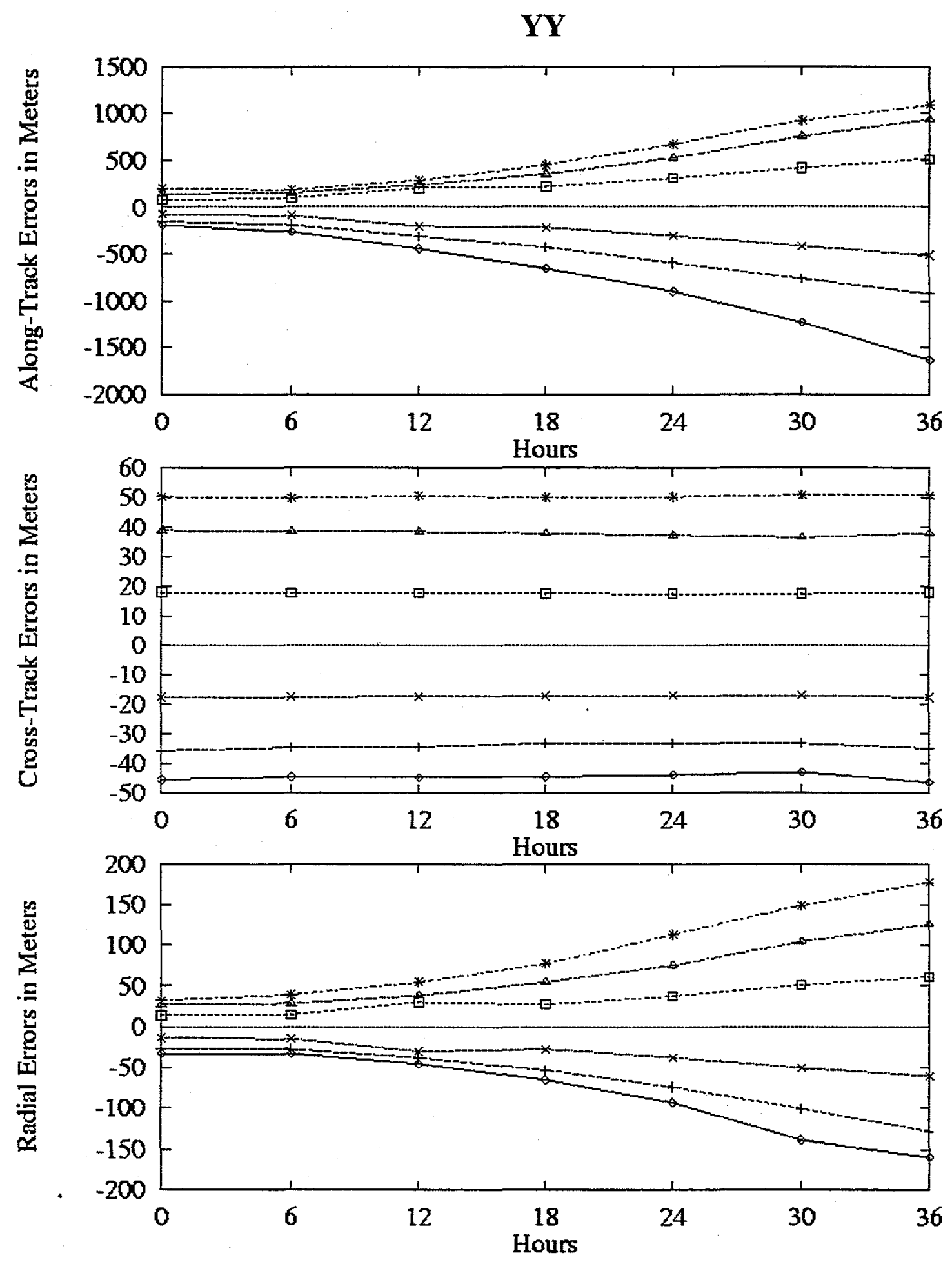


\section{ZZ}
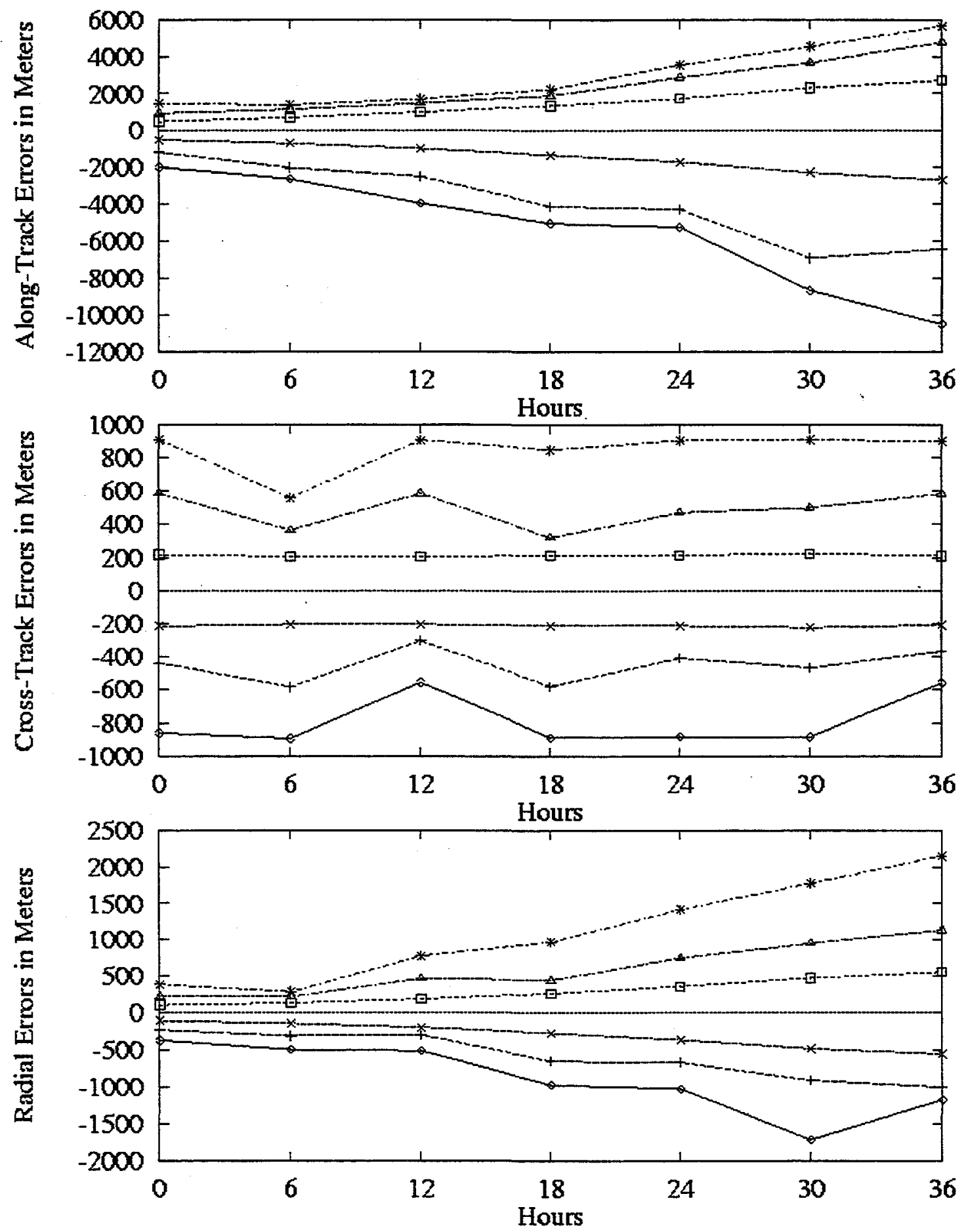


\section{1}
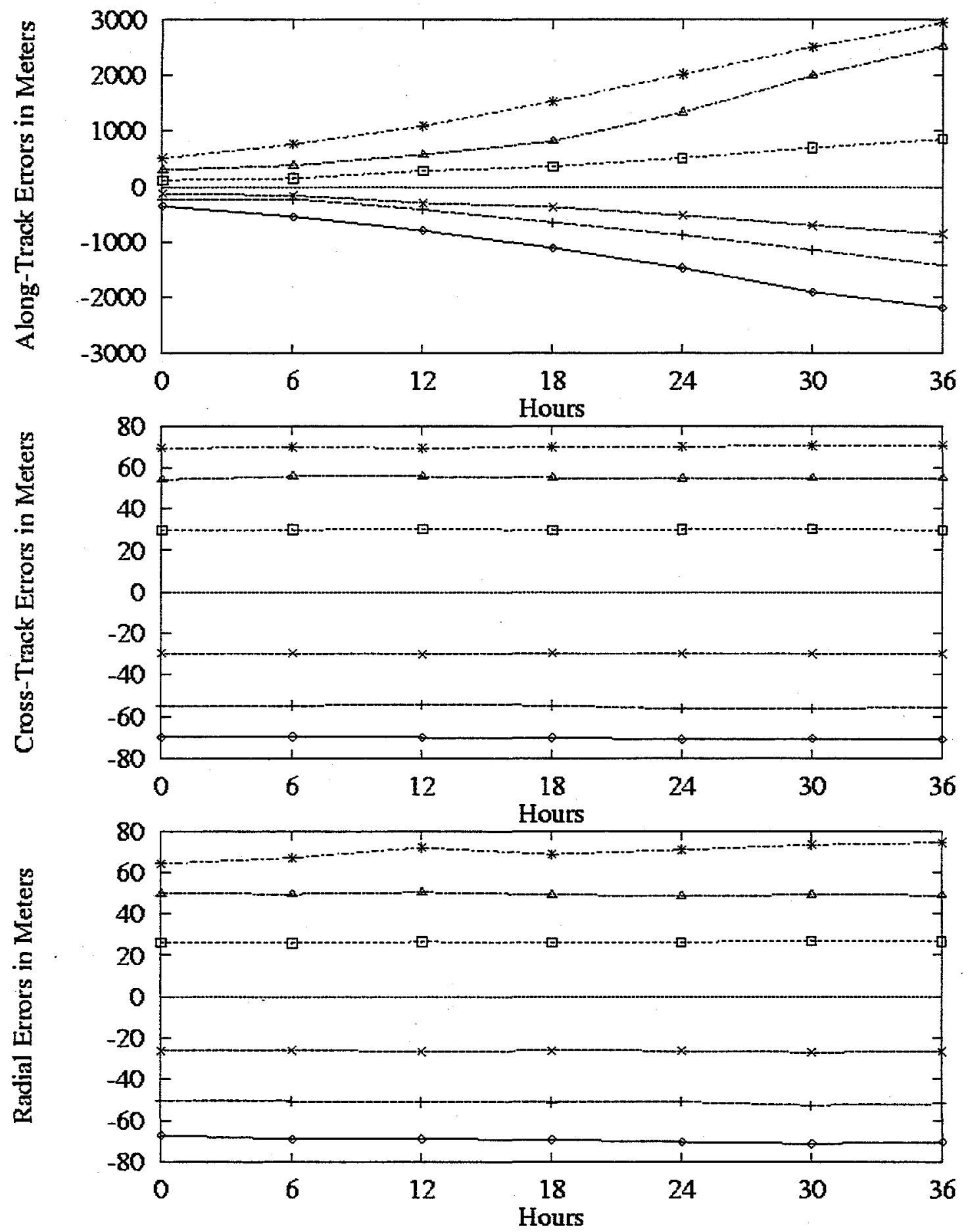
22
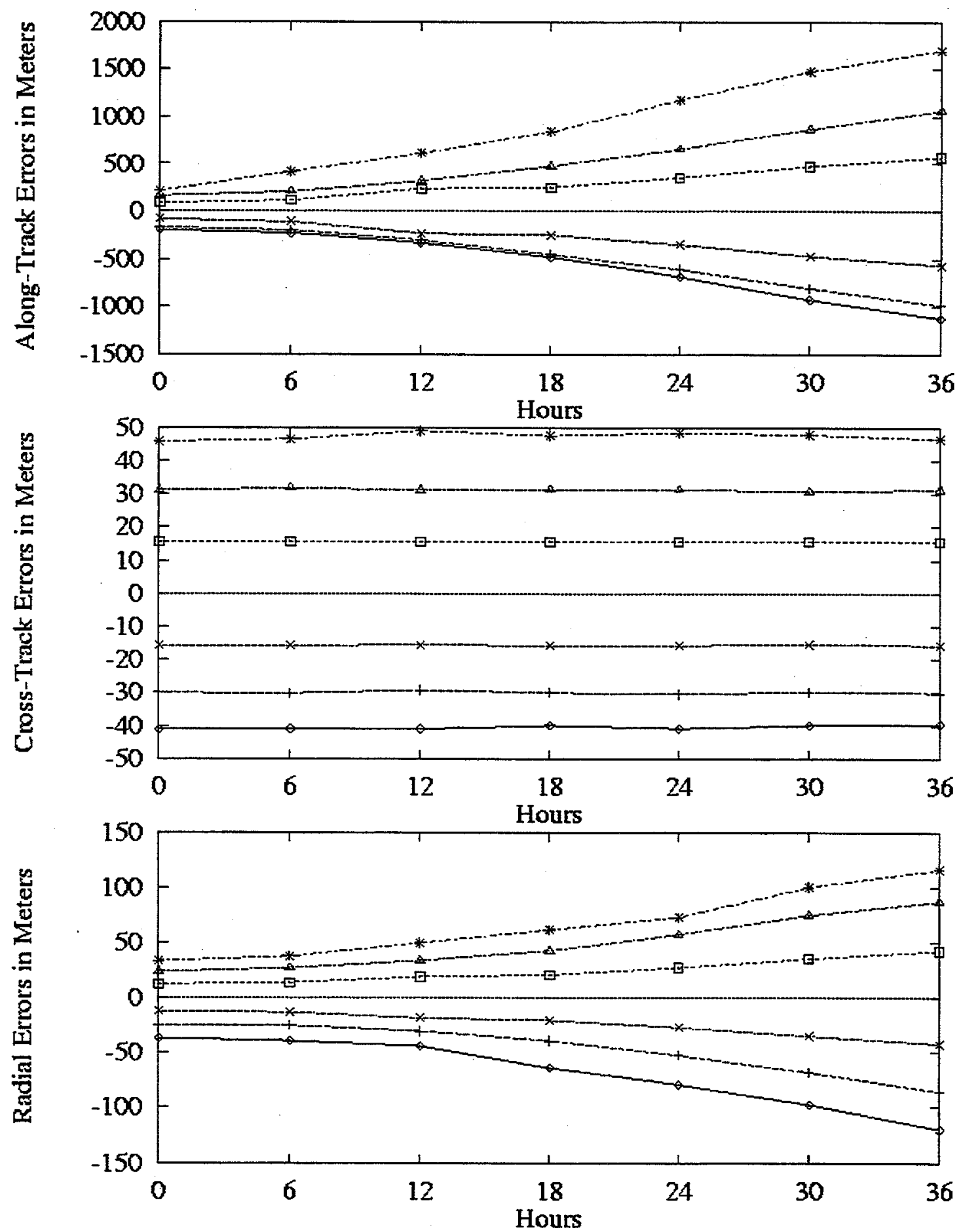
33
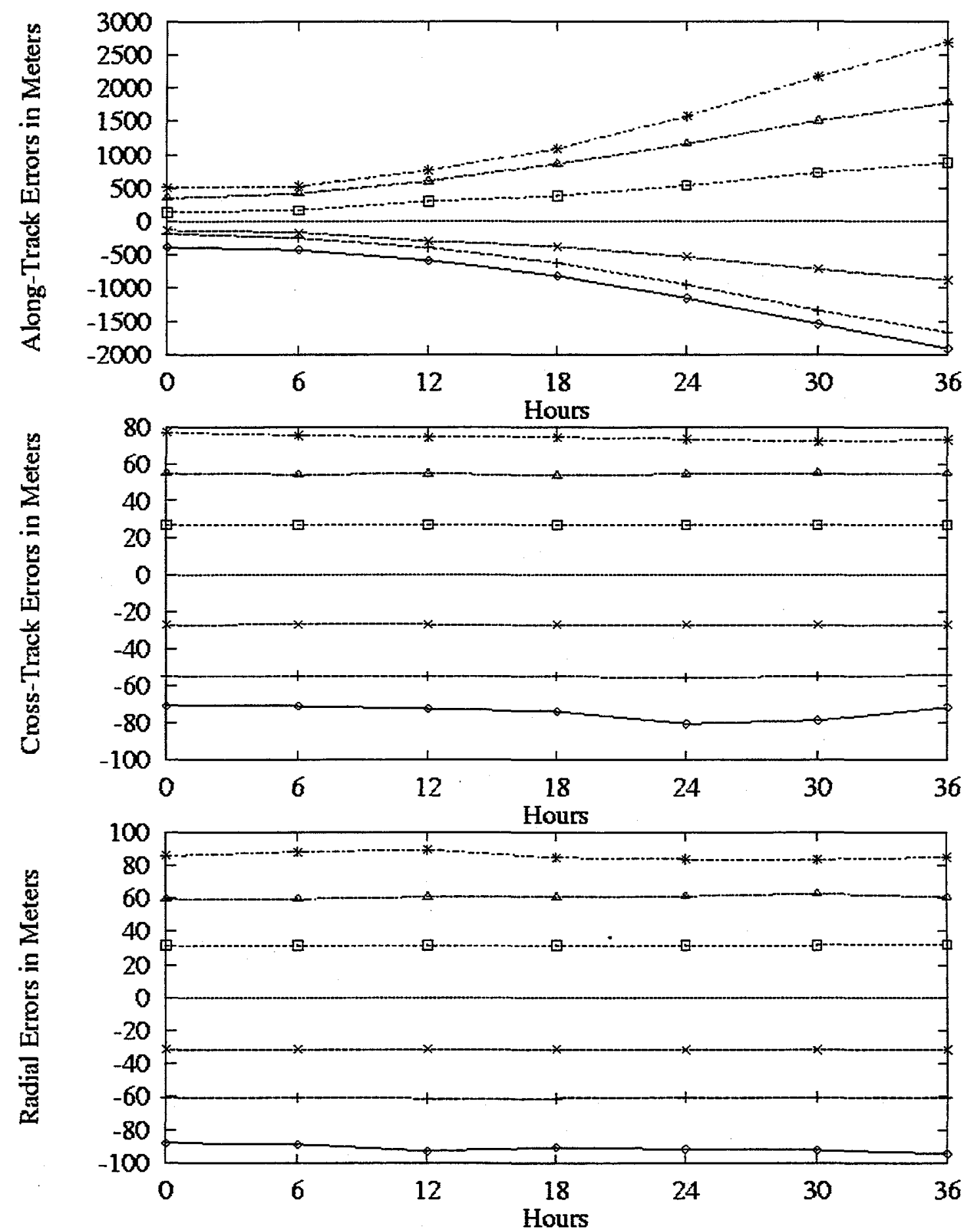
ORNL/TM-13726

\section{INTERNAL DISTRIBUTION}

1-5. M. Beckerman

6-10. J. P. Jones

11. M. R. Leuze

12. C. E. Oliver

13. W. A. Shelton
14. T. Zacharia

15. Laboratory Records - RC

16-17. Laboratory Records Dept./OSTI

18. Central Research Library

\section{EXTERNAL DISTRIBUTION}

19. Dr. Kyle T. Alfriend, Dept. of Aerospace Engineering, 722 H.R. Bright Building, Texas A\&M University, College Station, TX 77483-3141

20. Mr. W. N. Barker, ITT Systems and Sciences Corp., 4450 E. Fountain Blvd., Colorado Springs, CO 80933-7463

21. Mr. S. J. Casali, ITT Systems and Sciences Corp., 4450 E. Fountain Blvd., Colorado Springs, CO 80933-7463

22. Dr. Paul Cefola, Charles Stark Draper Laboratories, 555 Technology Square, Cambridge, MA 02139-3563

23. Dr. Shannon Coffey, Naval Research Laboratories Code 8233,4555 Overlook Ave S.E., Washington, D.C., 20375-5355

24. Mr. C. D. Cunningham, Department of Energy, P.O. Box 2001, MS 6269, Oak Ridge, TN 37831

25. DSRD Resource Center, Data Systems Research and Development, Lockheed-Martin Energy Systems, 1099 Commerce Park Drive, Oak Ridge, TN 37830

26. Mr. Dan Fonte, 3561 Cour Du Vin, San Jose, CA; 95148

27. Dr. J. Lee Foster, United Space Alliance, USH-423E, 600 Gemini, Houston, TX 77058

28. Mr. Joe Frisbee, United Space Alliance, USH-423E, 600 Gemini, Houston, TX 77058

29. Dr. C.W. Glover, POET/ORNL, Suite 1100, Arlington, VA 22202

30. Lt. Col. Darrell Herriges, 9240 Weathersfield Drive, Bristow, VA 20136

31. Dr. Nicholas L. Johnson, NASA Johnson Space Center, SN3, Houston, TX 77058

32. Dr. Jean de Lafontaine, Ecole de Technologie Superieure, 1100 rue Notre-Dame Ouest, Montréal (Québec), Canada H3C 1K3

33. Dr. J. J. F. Liu, SWC/AES, 730 Irwin Ave., Ste 83, Schriever AFB, CO, 80912-7383

34. Ms. Brenda Moulton, DM3/Orbit Branch, Flight Design and Dynamics Division, NASA Johnson Space Center, Houston, TX 77058

35. Dr. John O'Sullivan, POET/Aerospace, Suite 1100, Arlington, VA 22202

36. Mr. Al Perella, POET/IDA, Suite 1100, Arlington, VA 22202

37. Dr. Les Pierre, Director NMD BMC3I, BMDO/JNB, The Pentagon, Washington, DC 20301-7100

38. Dr. Ronald J. Proulx, Charles Stark Draper Laboratories, 555 Technology Square, Cambridge, MA 02139-3563

39. Mr. J. A. Reafsnyder, Department of Energy, P.O. Box 2001, MS M6, Oak Ridge, TN 37831

40. Mr. David B. Rowe, ESC Det 5/NDCC, 160 Patrick St., Peterson AFB, CO, 80914-2102 
41. Dr. Paul Schumacher, NAVSPACECOM/N6T3, 5280 $4^{\text {th }}$ St., Dahlgren, VA, 22448

42. Dr. David Spencer, Phillips Laboratories PL/VLSA, 3550 Aberdeen Ave. S.E., Kirtland AFB, NM, 87117

43. Dr. Ramaswamy Sridharan, MTT Lincoln Laboratories, 244 Wood St., Room S4-600, Lexington, MA, 02420-9185

44. Mr. D. J. Steele, ESC Det 5/NDCC, 160 Patrick St., Peterson AFB, CO, 80914-2102

45. Mr. Paul Temple, BMDO/AQE, The Pentagon, Washington, DC 20301-7100

46-61. R. G. Tudor, Data Systems Research and Development, Lockheed-Martin Energy Systems, 1099 Commerce Park Drive, Oak Ridge, TN 37830

62. Mr. Steve Waugh, BMDO/AQE, The Pentagon, Washington, DC 20301-7100

63. Lt. Col. D. Vallado, USSPC/AN, 250 S. Peterson Blvd, Ste 116, Peterson AFB, CO, 80914-3040

64. Mr. Dan Whitener, Dpeuty Director TMD BMC3I, BMDO/AQI, The Pentagon, Washington, DC 20301-7100

65. Mr. Michael B. Wortham, United Space Alliance, USH-423E, 600 Gemini, Houston, TX 77058 


\section{EXTERNAL REDISTRIBUTION}

66-111. Mr. Keith L. Nutt, ESC Det 5/NDCC, 160 Patrick St., Peterson AFB, CO, 80914-2102 SWC/AES, Attn: Dr. Joseph Liu, 730 Irwin Ave., Ste 83, Schriever AFB, CO, 80912-7383 CMOC/J3OXY, Attn: Maj Rabe, 1 NORAD Road, Ste 9-101, Cheyenne Mountain Air Station, CO 80914-6020

USSPC/AN, Attn: LtCol Vallado, 250 S. Peterson Blvd, Ste 116, Peterson AFB, CO 80914-3040 USSPC/J33O, Attn: Maj. Torres. 250 S. Peterson Blvd, Ste 116, Peterson AFB, CO 80914-3040 USSPC/J5R, Attn: Maj. Kendall, 250 S. Peterson Blvd., Ste 116, Peterson AFB, CO 80914-3080 AFSPC/DRC, Attn: Mr. Jim Reichman, 150 Vandenberg St., Ste 1105, Peterson AFB, CO $80914-4600$

AFSPC/DOY, Attn: Maj Gary Brandstrom, 150 Vandenberg St., Ste 1105, Peterson AFB, CO $80914-4220$

ITT Systems and Sciences Corp., Attn: Mr. Bill Barker, 4450 E. Fountain Blvd., P. O. Box 7463, Colorado Springs, CO 80933-7463

ESC Det 5/NDCC, Attn: Mr. Keith L. Nutt, 160 Patrick St., Peterson AFB, CO 80914-2102

NAVSPACECOM/N6T3, Attn: Dr. Paul Schumacher and LtCmdr Sonja Smith, $52804^{\text {th }}$ St., Dahlgren, VA 22448

Naval Research Laboratories, Attn: Dr. Shannon Coffey \& Mr. John Boors, NRL Code 8233, 4555 Overlook Ave S.E., Washington, D.C. 20375-5355

Air Force Research Laboratories, AFRL/VSSS, Attn: Dr. Rich Burns, 3550 Aberdeen Ave., S.E., Kirtland AFB, NM 87117-5776

NASA/Goddard Space Flight Center, Attn: Mr. Carl Smith, Mailstop 452.0, Greenbelt, MD 20771

MIT Lincoln Laboratories Attn: Dr. Ramaswamy Sridharan \& David Whited, 244 Wood St., Room S4-600, Lexington, MA 02420-9185

NASA Johnson Space Center/DM3, Attn: Ms Brenda Moulton, Orbit Br., Flight Design and Dynamics, Houston, TX 77058

Phillips Laboratories PL/VLSA, Attn: Dr. David Spencer, 3550 Aberdeen Ave. S.E., Kirtland $\mathrm{AFB}, \mathrm{NM} 87117$

Charles Stark Draper Laboratories, Attn: Dr. Paul Cefola \& Dr. Ronald J. Proulx, 555

Technology Square, Cambridge, MA 02139-3563 